\title{
Modulation of Conformational Exchange Events in the Glutamate Transporter Homologue Glt ${ }_{\mathrm{ph}}$
}

\author{
Sara Ann Blankenship \\ White Sulphur Springs, WV \\ Bachelor of Science, Chemistry; Roanoke College, 2012
}

\begin{abstract}
A Thesis presented to the Graduate Faculty of the University of Virginia in Candidacy for the Degree of

Doctor of Philosophy
\end{abstract}

Department of Chemistry

University of Virginia

August 2017 


\begin{abstract}
Solute transport, cell signaling, vesicle fusion, and many other important cellular functions are mediated by membrane proteins, which are coded for by approximately $30 \%$ of the genome and represent $60 \%$ of current drug targets. One important class of membrane proteins is the secondary active transporters, which mediate small molecule transport by harnessing the free energy stored in ion gradients. While there have been an increasing number of crystal structures of in recent years, secondary active transporters remain underrepresented structurally. Although crystal structures provide vital structural information, they do not always provide structures of biologically relevant conformations that are sampled during the transport cycle.

The solute carrier 1 family (SLC1) of secondary active transporters are responsible for neutral and charged amino acid transport, and defects in these transporters have been implicated in many central nervous systems and mental disorders such as Alzheimer's disease, epilepsy, obsessive/compulsive disorder, and schizophrenia. Glt $\mathrm{ph}_{\mathrm{ph}}$ is a sodium dependent aspartate transporter that is structurally homologous to the SLC1 family and the only member that has been crystallized. The series of crystal structures in substrate free, substrate bound, and inhibitor bound states have provided an outline of global conformational exchange events during the transport cycle. However, small-scale conformational exchange events that must mediate transport have remained uncharacterized. Furthermore, the impact of the lipid environment on conformational exchange has not been described.
\end{abstract}

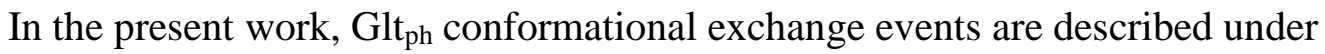
conditions that support transport. We propose a novel conformation for hairpin loop 1 
(HP1) that mediates substrate release intracellularly based on CW-EPR spectra. We also show that the protective osmolyte, sucrose, modulates conformational exchange differently in detergent micelles than in lipid bilayers based on DEER distance distributions, which has implications for the conformations seen in crystal structures. Furthermore, we show that the choice of spin label and lipids used in reconstitution modulate conformational populations. Finally, we propose, based on power saturation depth measurements, that the majority of $\mathrm{Glt}_{\mathrm{ph}}$ is buried in the lipid bilayer, in contrast to current structural models. The work presented here provides explanations for previous contrasting research and provides details on the transport cycle under biologically relevant conditions. 


\section{Contents}

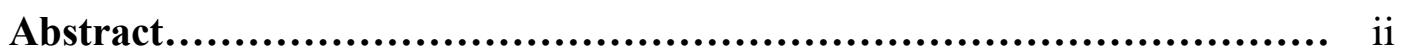

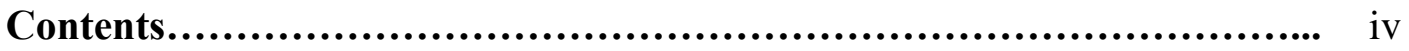

Figures.................................................................... vii

Tables........................................................................

Abbreviations............................................................. xii

Acknowledgements....................................................... xiv

Chapter 1: Membrane Proteins and the Environment........................ 1

1.1 The structure and function of membrane proteins..................... 2

1.2 The lipid bilayer's contribution to transporter structure and 6 function.................................................................

1.3 The effect of osmolytes on protein stability and conformational 9 exchange.................................................................

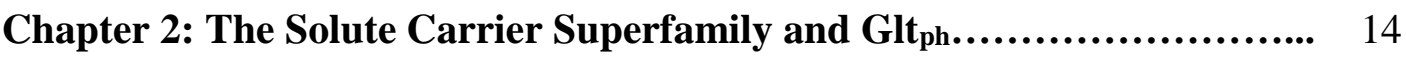

2.1 The Solute Carrier (SLC) Superfamily .............................. 15

2.2 The glutamate transporter homologue Glt $_{\mathrm{ph}} \ldots \ldots \ldots \ldots \ldots \ldots \ldots \ldots \ldots \ldots . . . \ldots \ldots$

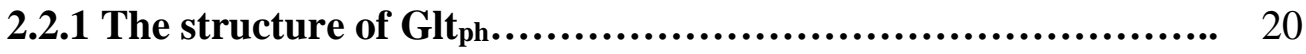

2.2.2 The elevator model.............................................. 23

2.2.3 $\mathrm{Na}^{+}$and aspartate coupling................................... 24

2.2.4 Thermodynamics and kinetics of conformational exchange...... 25

2.2.5 Intermediate conformations and influence of the lipid bilayer... 26 
Chapter 3: EPR Theory and Applications................................. 29

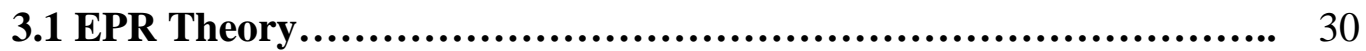

3.1.1 The resonance condition........................................ 30

3.1.2 The g-tensor................................................... 31

3.1.3 The A-tensor................................................... 31

3.1.4 Relaxation...................................................... 32

3.1.5 Continuous Wave EPR............................................ 34

3.1.6 Power Saturation.............................................. 35

3.1.7 Double Electron-Electron Resonance......................... 35

3.2 EPR Applications and Interpretation.............................. 39

3.2.1 Site Directed Spin Labeling................................... 39

3.2.2 CW-EPR...................................................... 40

3.2.3 Power Saturation................................................. 46

3.2.4 DEER............................................................. 47

Chapter 4: Results........................................................... 51

4.1 Introduction....................................................... 52

4.2 The inward facing conformation is stabilized by substrate in lipid 53 bilayers.

4.3 The conformationally restricted spin label, R1p, stabilizes the 60 inward facing conformation..........................................

4.4 Accessibility and depth measurements show unexpected protein/ 63 lipid headgroup contact..............................................

4.5 Lipid headgroups modulate intermediate or asymmetric 69 conformations..............................................................

4.6 The $\mathbf{R} 1$ side chain alters conformational populations in detergent $\quad 75$ micelles. 
4.7 HP1 samples conformations not observed in crystal structures...... 77

4.8 Chloride alters conformational populations....................... 82

Chapter 5: Discussion and Concluding Remarks.......................... 86

Chapter 6: Appendix...................................................... 95

Chapter 7: Materials and Methods.......................................... 109

Chapter 8: References........................................................ 115 


\section{Figures}

1.1 Schematic of membrane proteins in a lipid bilayer...................... 2

1.2 Two examples of common secondary active transporter folds.......... 4

1.3 Conformations sampled during the alternating access mechanism...... 5

1.4 The effect of hydrophobic mismatch on the lipid bilayer............... 7

1.5 Intrinsic lipid and membrane curvature.............................. 8

1.6 Stabilization of the native protein fold by protective osmolytes......... 10

1.7 Contributions to transfer free energy of the protein backbone and 11 side chains.

1.8 Probing conformational exchange events in the Ton box of BtuB using the protective osmolyte polyethylene glycol (PEG).

2.1 Genes encoding human transporter proteins

2.2 Cumulative number of crystal structures of SLC1 family members..... 16

2.3 Overview of glutamatergic signaling................................... 19

2.4 Sequence homology of GIt ph $_{\text {with three synaptic glutamate } 20}$ transporters.................................................................

2.5 Crystal structures of the outward and inward facing conformations 21 of Glt ph $_{\text {. }}$

2.6 Gate movements of HP1 and HP2 in the inward facing conformation.

2.7 The alternating access/elevator model of transport.

2.8 The outward and inward facing conformations have nearly equal free energies.

2.9 Transport domain conformational heterogeneity in detergent micelles and POPC bilayers. 
3.2 Loss and reestablishment of phase coherence........................ 37

3.3 The site directed spin labeling reaction with MTSL.................... 40

3.4 A summary of the most common EPR experiments.................... 41

3.5 Secondary structure as determined by the second moment and the 42 inverse central linewidth...................................................

3.6 Characteristic lineshapes for loop, solvent exposed alpha helical, and 43 buried sites.

3.7 Alpha helical lineshapes in a hydrophobic environment............... 44

3.8 A two component CW-EPR spectrum from conformational exchange... 45

3.9 Power saturation schematic.......................................... 47

3.10 Subtraction of the background from the DEER dipolar evolution and 48 the resulting form factor..............................................

3.11 The effect of the regularization parameter on $\mathbf{P}(\mathbf{r}) \ldots . . . \ldots . . . \ldots \ldots . . . . \quad 49$

4.1 DEER distance distribution for V176T1 in the trimerization domain... 54

4.2 CW-EPR spectra for V176R1 ........................................ 54

4.3 $\mathrm{C}_{\alpha}-\mathrm{C}_{\alpha}$ distances for $\mathrm{T375}$ in the outward and inward facing 55 conformations..................................................................

4.4 DEER distance distributions and rotamer library analysis for T375R1 57

4.5 Substrate-free and $\mathrm{Na}^{+}$bound distance distributions for T375R1 60 compared to rotamer library analysis of the asymmetric crystal structure.

4.6 DEER distance distributions for T375R1p......................... 61

4.7 CW-EPR spectra for T375R1 and T375R1p......................... 63

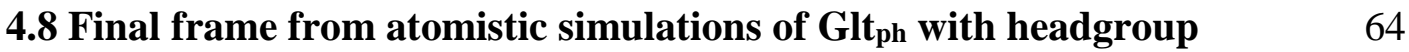

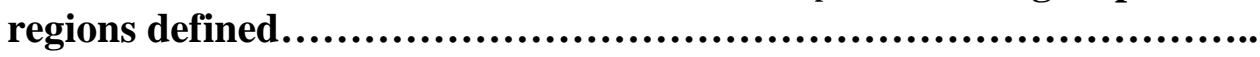

4.9 Membrane topology map for select Glt ph $_{\text {sites in the outward and } 65}$ inward facing conformations. 
4.10 Depth measurements for select sites in the 3-4 loop, transport 66 domain, and trimerization domain....................................

4.11 CW-EPR spectra for Q121R1, K125R1, and P128R1.................. 67

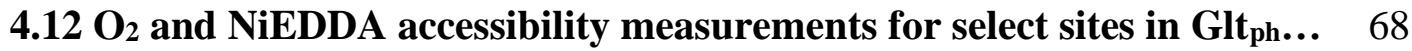

4.13 CW-EPR spectra for T166R1 and V176R1......................... 69

4.14 CW-EPR spectra for V176R1 in 3:1 E. coli polar lipids:POPE or 70 3:1 E. coli polar lipids:POPC...........................................

4.15 CW-EPR spectra for T166R1 in 3:1 E. coli polar lipids:POPE or 71 3:1 E. coli polar lipids:POPC........................................

4.16 DEER distance distributions for T375R1 in different lipid systems.... 73

4.17 DEER distance distributions for T375R1 in different lipid systems 74 with $30 \%$ sucrose added................................................

4.18 DEER distance distributions for T375R1 in detergent micelles........ 76

4.19 Crystal structures of Glt ph $_{\text {in }}$ the outward and inward facing 78 conformations with HP1 highlighted

4.20 CW-EPR spectra at select sites adjacent to substrate coordinating $\quad 79$ residues in HP1..................................................................

4.21 CW-EPR spectra for select sites at the apex of HP1 with and without 80 $30 \%$ sucrose

4.22 CW-EPR spectra for S277R1 and S279R1 with and without 30\% sucrose.

4.23 CW-EPR spectra for T166R1 with chloride or gluconate 83

4.24 CW-EPR spectra for T375R1 with chloride or gluconate.............. 84

4.25 CW-EPR spectra for K125R1 with chloride or gluconate............. 85

A.1 DEER distance distributions for select transport domain sites.......... 96

A.2 Aspartate uptake of select transport domain sites compared to wild 97 type control before and after spin labeling.............................

A.3 CW-EPR spectra for Gltph K125R1................................ 97 
A.4 DEER distance distribution reliability for T375R1 in 3:1 E. coli 98 polar lipids:POPE liposomes..........................................

A.5 DEER distance distribution reliability for T375R1 in 3:1 E. coli 99 polar lipids:POPE liposomes with $30 \%$ sucrose

A.6 DEER distance distribution reliability for T375R1p in 3:1 E. coli 100 polar lipids:POPE liposomes.

A.7 DEER distance distribution reliability for T375R1p in 3:1 E. coli 101 polar lipids:POPE liposomes with $30 \%$ sucrose

A.8 DEER distance distribution reliability for T375R1 in 3:1 E. coli 102 polar lipids:POPC liposomes

A.9 DEER distance distribution reliability for T375R1 in 3:1 E. coli 103 polar lipids:POPC liposomes with $30 \%$ sucrose

A.10 DEER distance distribution reliability for T375R1 in 3:1:1 DOPE: DOPC:DOPG liposomes.

A.11 DEER distance distribution reliability for T375R1 in 3:1:1 DOPE: DOPC:DOPG liposomes with $30 \%$ sucrose

A.12 DEER distance distribution reliability for T375R1 in DDM 106 detergent micelles

A.13 DEER distance distribution reliability for T375R1 in DDM detergent micelles with $\mathbf{3 0 \%}$ sucrose.

A.14 Calibration curve used for the Power Saturation experiments

\section{Tables}

2.1 The SLC1 Transporter Family

3.1 Resolution of DEER distributions at different dipolar evolution times (d2)

4.1 Mean distances and peak widths for DEER distance distributions at 56 V176R1, T375R1, and T375R1p.

4.2 Conformational populations based on distance distributions at T375R1....

4.3 Spin label rotamers and solvent accessible surface area (SASA) at 78 selected residues along the apex of HP1 


\section{Abbreviations and Symbols}

$\left\langle\mathrm{H}^{2}\right\rangle^{-1}$

$\AA$

Ala (A)

Apo

$\operatorname{Arg}(\mathrm{R})$

Asn $(\mathrm{N})$

Asp (D)

ATP

CW

Cys (C)

d2

DDM

DEER

DL-TBOA

DNA

DOPC

DOPE

DOPG

E. coli

EPR second moment

angstrom

alanine

substrate free

arginine

asparagine

aspartate

adenosine triphosphate

continuous wave

cysteine

dipolar evolution time

n-dodecyl- $\beta$-D-maltoside

double electron-electron resonance

DL-threo-benzyloxyaspartate

deoxyribonucleic acid

1,2-dioleoyl-sn-glycero-3-phosphocholine

1,2-dioleoyl-sn-glycero-3-phosphoethanolamine

1,2-dioleoyl-sn-glycero-3-phospho-(1'-rac-glycerol)

Escherichia coli

electron paramagnetic resonance 
FRET

fSASA

$\operatorname{Gln}(\mathrm{Q})$

Glu (E)

HP1

HP2

$\mathrm{K}_{\mathrm{D}}$

Lys (K)

MFS

MMM

MTSL

$\mathrm{Na}^{+} /$Asp

NiEDDA

NMR

$\mathrm{P}$

PC

PE

PEG

PG

PL fluorescence resonance energy transfer fractional solvent exposed surface area glutamine glutamate hairpin loop 1 hairpin loop 2 dissociation constant lysine major facilitator superfamily Multiscale Modeling of Macromolecules (1-Oxyl-2,2,5,5-tetramethylpyrroline-3-methyl) methanethiosulfonate sodium and aspartate nickel ethylenediaminediacetic acid nuclear magnetic resonance power phosphatidylcholine phosphatidylethanolamine polyethylene glycol phosphatidylglycerol polar lipids 
POPC

POPE

Pro (P)

R1

R1p

SASA

SDSL

$\operatorname{Ser}(\mathrm{S})$

SLC

$\mathrm{T}_{1}$

$\mathrm{T}_{2}$

TEMPO-PC

Thr (T)

TM

$\mathrm{T}_{\mathrm{m}}$

TMAO

Val (V)

$\Delta \mathrm{G}$

$\Delta \mathrm{H}^{-1}$

$\Phi$ 1-palmitoyl-2-oleoyl-sn-glycero-3-phosphocholine

1-palmitoyl-2-oleoyl-sn-glycero-3-phosphoethanolamine

proline

MTSL attached to cysteine

3-methanesulfonilthiomethyl-4-(pyridin-3-yl)-2,2,5,5-tetramethyl2,5-dihydro-1H-pyrrol-1-yloxyl solvent exposed surface area site directed spin labeling serine solute carrier family longitudinal relaxation transverse relaxation 1,2-dipalmitoyl-sn-glycero-3-phospho(tempo)choline

threonine transmembrane helix echo decay time trimethylamine-N-oxide valine Gibbs free energy inverse central linewidth depth parameter 


\section{Acknowledgements}

God, for the brain to do this

My parents, Sue and Marty, for instilling the correct amount of stubbornness

My fiancé, Arthur, for making sure I don't starve when I decide to work way too long

Marissa and Jason, for being my sanity by going on lots of lunches with me

Dr. David Cafiso, for his advice, guidance, and being a really good sport in the face of sarcasm

My lab, for being an unending source of both project ideas and entertainment

Dr. Frackson Mumba, for his Chemical Education mentoring and advice about lawn care 
Chapter 1

Membrane Proteins and the Environment 


\subsection{The structure and function of membrane proteins}

Solute transport, cell signaling, vesicle fusion, and many other important cellular functions are mediated by membrane proteins, which are coded for by approximately $30 \%$ of the genome and represent $60 \%$ of current drug targets (Lodish et al., 2000; Stevens and Arkin, 2000; Hopkins and Groom, 2002). Despite their importance, membrane proteins are underrepresented structurally due to difficulties in expression, purification, and retention of native-like structure and activity (Tan et al., 2008). While the development of new expression systems and purification tags have increased protein yield, it is impossible for membrane mimetics to replicate the heterogeneous, dynamic, and crowded native membrane environment (Figure 1.1). Membrane proteins are classified as integral or peripheral, depending on their specific interactions with the lipid bilayer. Integral membrane proteins are composed of highly hydrophobic $\alpha$-helices or $\beta$ sheets that span the lipid bilayer, while peripheral membrane proteins either interact with integral membrane proteins or the lipid headgroups (Figure 1.1) (Lodish et al., 2000).

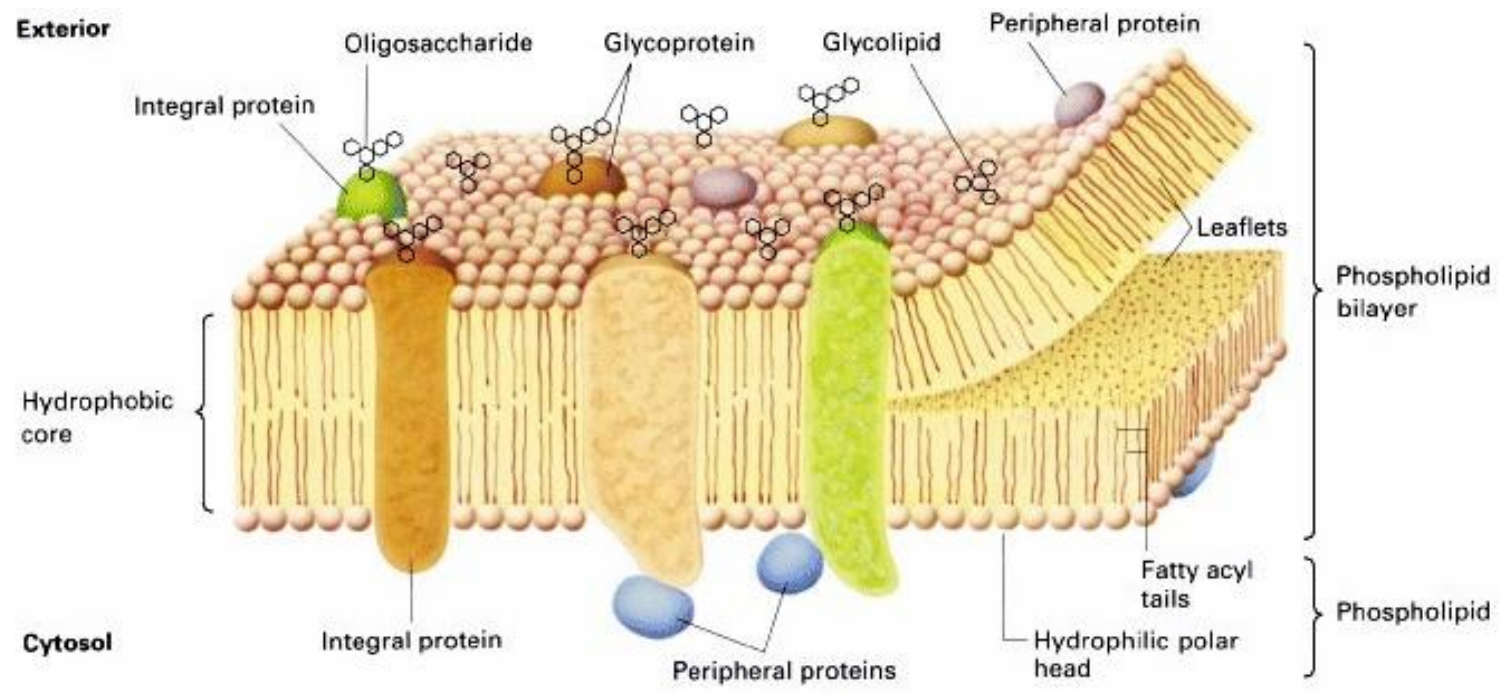

Figure 1.1. Schematic of membrane proteins in a lipid bilayer. (Lodish et al., 2000) Integral membrane proteins span the bilayer, while peripheral proteins interact with integral membrane proteins near the aqueous/bilayer interface. 
Integral membrane proteins control the flow of information through receptor signal transduction or the movement of molecules through transporters, which are broadly dividing into four groups (Whitelegge, 2013). Channels do not require an energy source and are diffusion controlled. Primary active transporters are coupled to a primary energy source, such as ATP hydrolysis, while secondary active transporters are coupled to an electrochemical gradient, such as $\mathrm{Na}^{+}$or $\mathrm{H}^{+}$. Group translocators alter substrate during transport (Ken \& Pauisers, 2005). Secondary active transporters are further subdivided. Uniporters transport one molecule, while symporters transport ions with substrate. Antiporters countertransport ions after substrate movement, and exchangers countertransport another similar substrate (Saier, 2000). Many transporters combine multiple modes of action through the transport cycle.

Despite low sequence homology among families, secondary active transporters share common structural elements and overall transport mechanisms (Shi, 2013). Structural commonalities include pseudosymmetry, which usually manifests as inverted topological repeats, and discontinuous or broken helices, which always occur in pairs and either span the membrane or are reentrant hairpins (Screpanti and Hunte, 2007). There are multiple common folds that all share these two defining characteristics, such as the Major Facilitator Superfamily (MFS), LeuT, and Gltph folds, which are the most common (Figure 1.2) (Shi, 2013). The transport mechanism is the alternating access model, which is defined by large scale conformational changes that allow substrate binding on one side of the membrane and substrate release on the other side without allowing both sides to be exposed simultaneously (Jardetzky, 1966). 

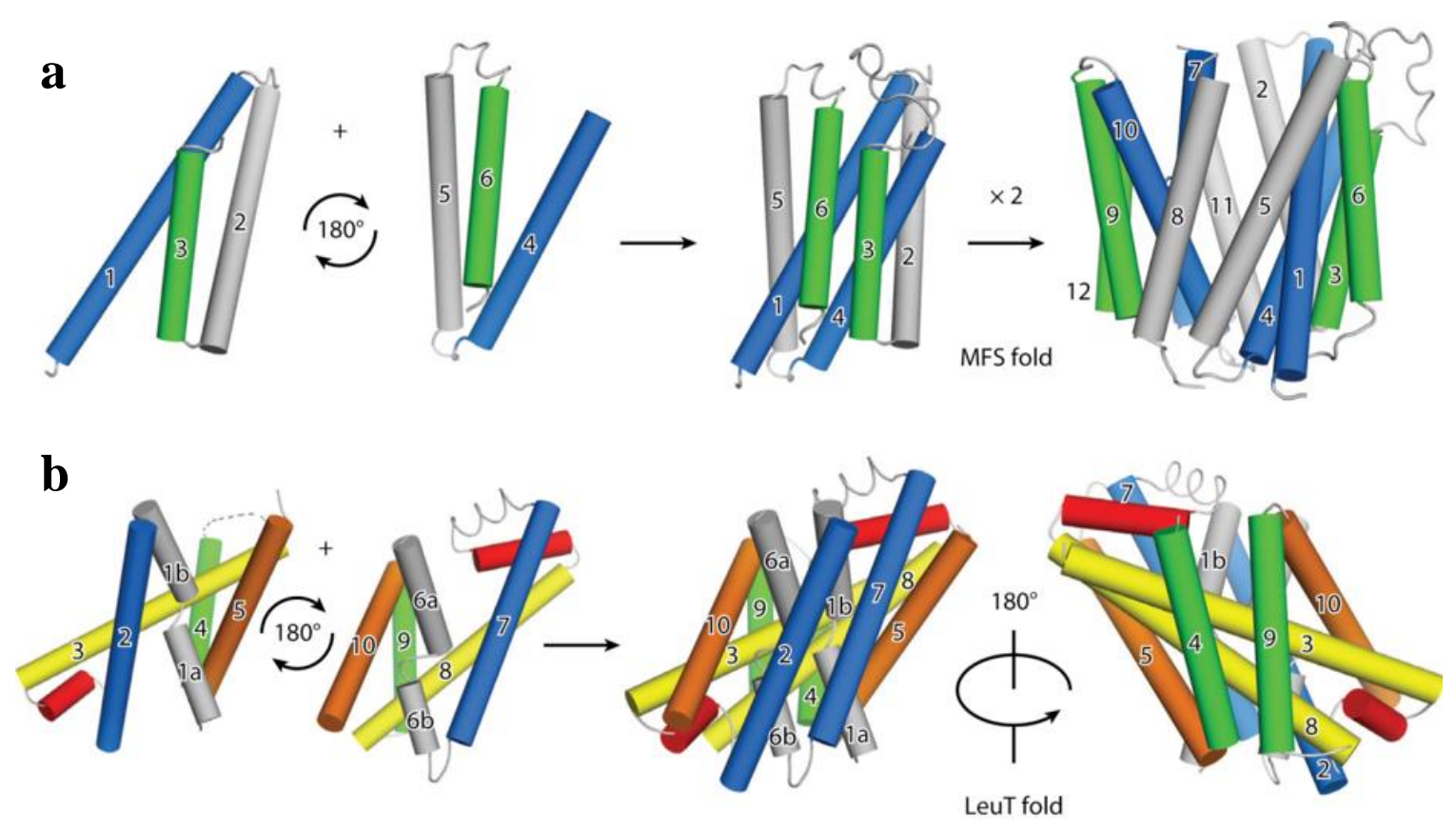

Figure 1.2. Two examples of common secondary active transporters folds. Both share inverted topology repeats and broken helices. a) The Major Facilitator Superfamily (MFS) fold, which contains two pairs of inverted repeats. b) The LeuT fold, which contains one pair of inverted repeats. (Adapted from Shi, 2013).

This process is controlled by a sequence of open and occluded states that allow substrate binding, release, and transporter reorientation through the opening and closing of extracellular and intracellular gates. (Figure 1.3) (Forrest et al., 2010). For the MFS and LeuT folds, the gates are not part of the substrate binding site. Under this model, after substrate binds, the transporter must move through a high energy intermediate state. This is driven by conformational changes that occur once substrate is bound. The conformational changes reduce the energy penalty of intermediates, allowing transport to occur (Figure 1.3) (Forrest et al., 2010). Once substrate is released intracellularly, the empty transporter must reorient the substrate binding site to the extracellular side, which is partially driven by the downhill ion gradient (Forrest et al., 2010). However, for the Glt $\mathrm{ph}_{\mathrm{f}}$ fold, both the extracellular and intracellular gates form the substrate binding site, which alters the energy of the substrate bound and intermediate states (Yernool et al., 
2004). The energy from substrate binding induced conformational changes is needed to keep the extracellular gate closed, and transitions to the intracellular side are driven by thermal energy alone (Forrest et al., 2010). This has been verified experimentally as Glt $\mathrm{ph}_{\mathrm{ph}}$ has been shown to sample both extracellular and intracellular conformations when no substrate is bound (Hanelt et al., 2013; Georgieva et al., 2013; Akyuz et al., 2015).

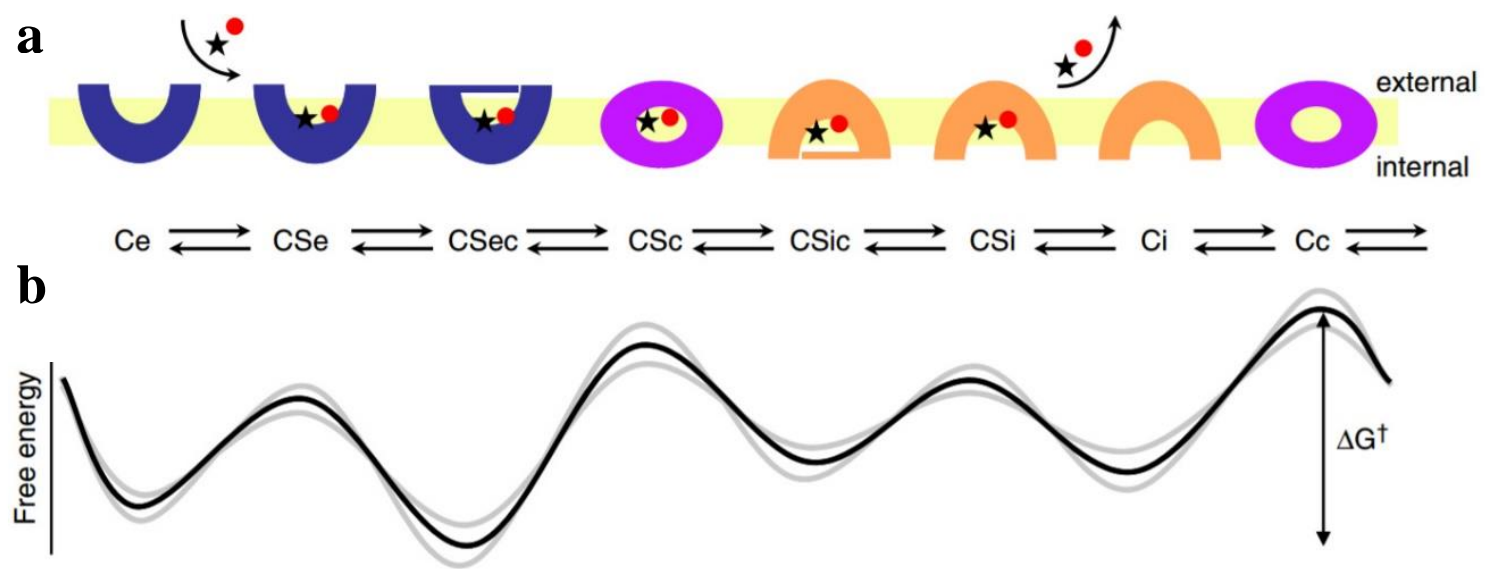

Figure 1.3. Conformations sampled during the alternating access mechanism. A) A series of open, substrate bound, and occluded states that must occur during transport. B) The energy landscape of conformational changes. There are multiple high energy intermediates that have been difficult to observe experimentally. (Adapted from Forrest et al., 2010)

Crystal structures of common folds show variations of the alternating access model that are shared among proteins that have the same common fold (Shi, 2013). However, variations of the higher energy intermediate states are difficult to capture crystallographically as proteins are trapped in an energy minimum under the conditions needed to crystalize a protein. Furthermore, in native systems, the lipid bilayer and solute exclusion from macromolecular surfaces affect conformational states that can be sampled during the transport cycle. 


\subsection{The lipid bilayer's contributions to transporter structure and function}

The lipid bilayer is a liquid crystalline environment with chemical and dielectric gradients that modulate transporter rates and conformational equilibria. The center of the bilayer has a dielectric constant of 2-4 that increases gradually toward the headgroup region (Cross et al., 2014). The bulk aqueous phase has a dielectric constant of approximately 80 , and the aqueous/bilayer interface has a dielectric constant that can reach 200 due to charges on the lipid headgroups (Cross et al., 2014). Membrane protein sequences typically reflect this large dielectric gradient with hydrophobic residues facing the bilayer and charged residues near the headgroups. Tryptophan and tyrosine are also typically found in the headgroup region as they anchor proteins through hydrogen bonding and cation/pi interactions with the headgroups and their planar shape (Sun et al., 2008). The bilayer, once thought of as only a hydrophobic solvent, is now known to have both general and specific interactions with membrane proteins. Physical and chemical properties of the bilayer, such as hydrophobic thickness and headgroup composition, are known modulators of protein stability and function (Mouritsen and Bloom, 1984; Gruner, 1985).

The hydrophobic thickness of the lipid bilayer may alter the conformations a membrane protein can sample through a hydrophobic mismatch, which occurs when the bilayer's hydrophobic thickness does not match the hydrophobic length of the protein. This leads to an energy penalty for certain protein conformational states due to aqueous exposure of hydrophobic residues (Andersen and Koeppe, 2007). Deformations around the protein-lipid interface can allow localized changes in hydrophobic thickness (Figure 1.4). 


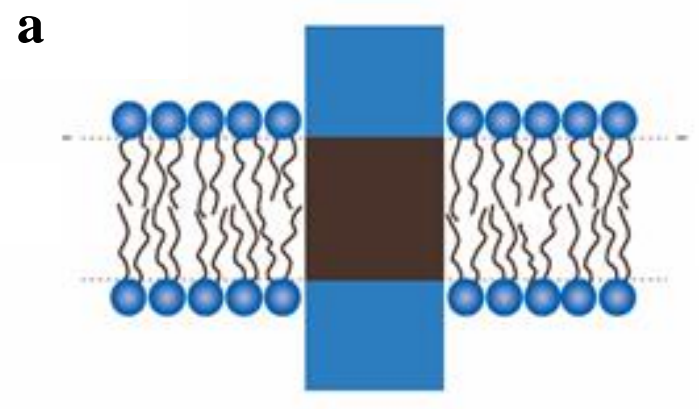

b

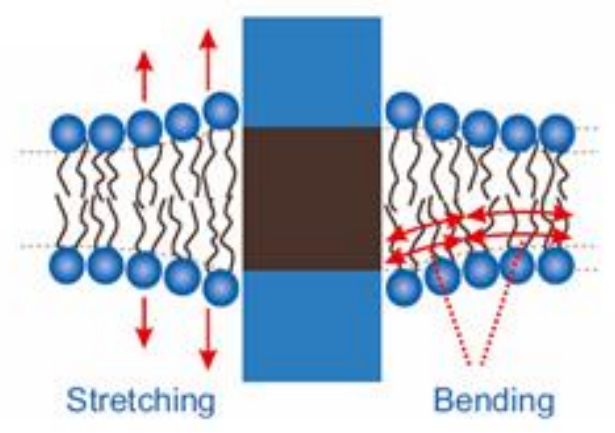

c

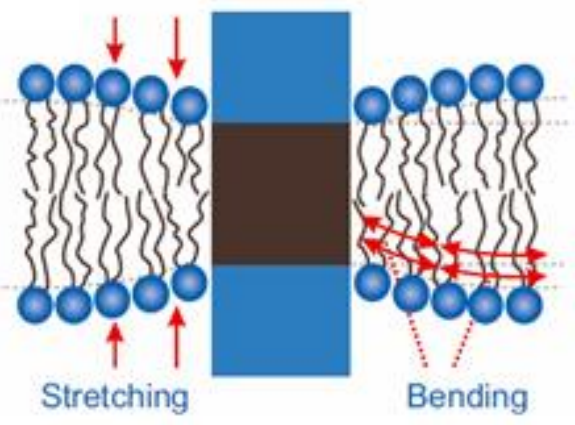

Figure 1.4. The effect of hydrophobic mismatch on the lipid bilayer. A) A hydrophobic match between the hydrophobic length of the protein and the surrounding bilayer. B and C) Hydrophobic mismatch where the hydrophobic length of the protein is longer or shorter than the hydrophobic thickness, respectively. The hydrophobic residues are represented by the gray box. (Adapted from Olaf and Koeppe, 2007) .

However, protein conformational states that minimize mismatch are energetically favored. Even small hydrophobic differences between the protein and surrounding bilayer can constrain the energy landscape available during transport (Andersen and Koeppe, 2007).

Lipid headgroups modulate both general and specific interactions with transporters. Lipids have intrinsic curvature that is determined by the relation between the area of the headgroup and the lipid tails, and this controls the lipid's propensity to form bilayer or nonbilayer structures (Figure 1.5) (Andersen and Koeppe, 2007). 
Lipids such as phosphatidylcholine (PC) and phosphatidylglycerol (PG) have a headgroup and tail region with similar areas, so they have a cylindrical shape and tend to form bilayers with no curvature strain. Positive curvature is induced by lipids with a

\section{Lyso-lipids}

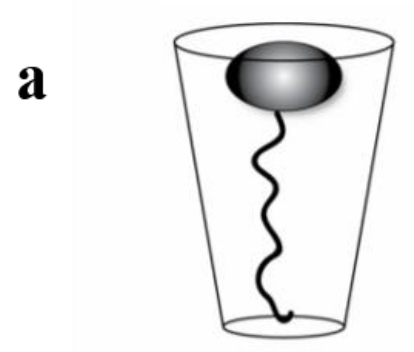

NON-BILAYER PRONE

POSITIVE CURVATURE
PC, PG

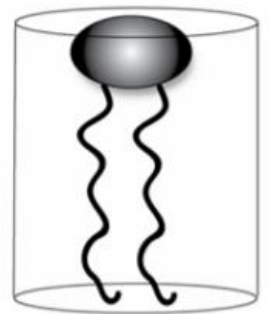

BILAYER PRONE

ZERO CURVATURE

b
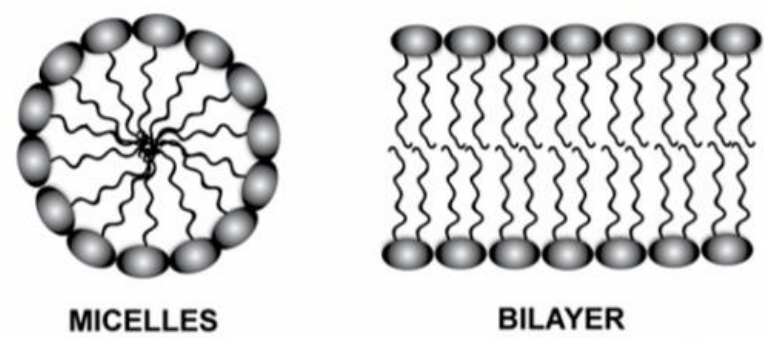

BILAYER

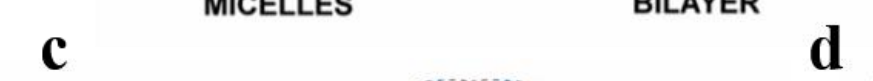

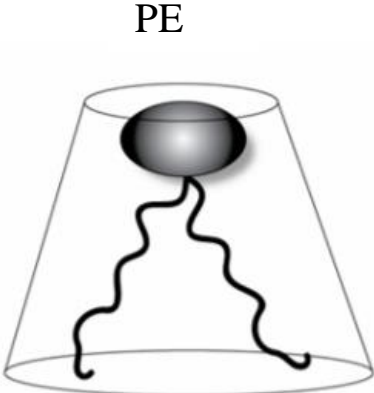

NON-BILAYER PRONE

NEGATIVE CURVATURE
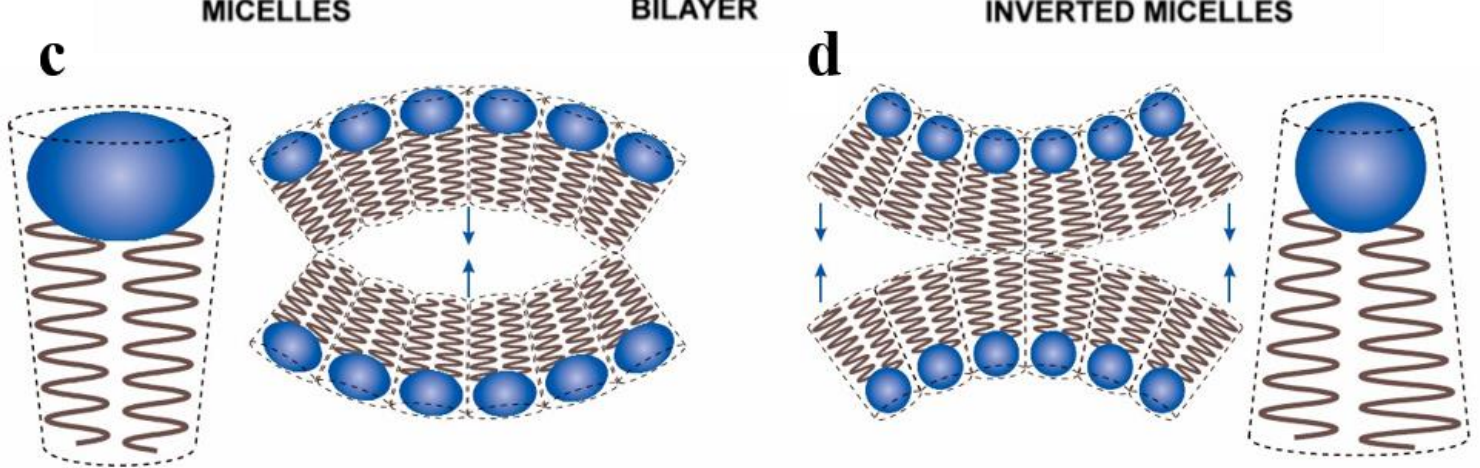

Figure 1.5. Intrinsic lipid and membrane curvature. a) Bilayer and nonbilayer prone lipids. Lipids with headgroups and tail regions with similar areas are cylindrical and form bilayers with no curvature, while lipids with differences in head to tail areas are nonbilayer prone. b) Structures formed in solution by lipids with positive, zero, and negative curvature, respectively. $\mathrm{c}$ and d) Lipids with positive intrinsic curvature and negative intrinsic curvature form structures that curve to reduce bilayer frustration; positive curvature pushes the headgroups toward the extracellular side, and negative curvature forms inverted bilayers (Adapted from van den Brink et al., 2004; Brown, 2012). 
larger headgroup than tail region, which is typically seen in the single chain lyso lipids. Lipids with small headgroups such as phosphatidylethanolamine (PE) induce negative curvature (Figure 1.5) (Brown, 2012). Membrane curvature changes lateral pressure, which can modulate protein function (van den Brink et al, 2004).

Specific interactions with annular and nonannular lipids also contribute to protein stability and energy landscape during the transport cycle. Annular lipids are the first shell of lipids around the perimeter of the protein and have been shown to regulate the function of channels and mechanosensitive proteins (Powl et al., 2007; Marius et al., 2005). These are typically negatively charged lipids such as PG or cardiolipin that interact with pairs or a series of positively charged residues (Powl et al., 2008; Lee, 2011). Nonannular lipids are typically trapped within the protein structure and can regulate protein folding and conformational exchange events (Lee, 2011). These are stabilized through electrostatic or hydrogen bonding headgroup interactions with pairs of positively charged or aromatic residues, which necessarily excludes PC as a nonannular lipid (Palsdottir, 2004). Switching PC for PE for vesicle reconstitution has been shown to increase transport activity in the glutamate transporter homologue, Gltph (McIlwain et al., 2015).

\subsection{The effect of osmolytes on protein stability and conformational exchange}

Osmolytes are small molecules that protect cells and cellular contents from environmental stresses such as temperature fluctuations, solute concentration fluctuations, and hydrostatic pressure. They also modulate macromolecular folding and interactions (Bolen, 2001). The two groups of osmolytes are denaturing osmolytes such as urea, and protecting osmolytes such as polyols (e.g. glycerol and sucrose), amino acids, and methylamines (e.g. trimethylamine-N-oxide (TMAO)). Since osmolytes act on 
different types of macromolecules, the effect on protein stability must be nonspecific (Harries, 2008). Hence, most organisms use organic osmolytes instead of salts, since salts interact electrostatically with macromolecular surfaces (Tanford, 1957).

Protecting osmolytes have been shown to stabilize protein folding, promote ligand and DNA binding, and modulate conformational energetics through preferential exclusion of the osmolyte from the protein surface. This was demonstrated by dialysis experiments that showed sucrose favored the dialysis compartment that did not contain protein (Pace and Shaw, 2000; Baskakov and Bolen, 1998; Parsegian et al., 1995; Smith, 2006). Gibbs free energy transfer experiments showed a greater free energy increase for denatured protein transferred to $1 \mathrm{M}$ protective osmolyte than for folded protein (Figure 1.6).

\section{Stabilizing Osmolytes}
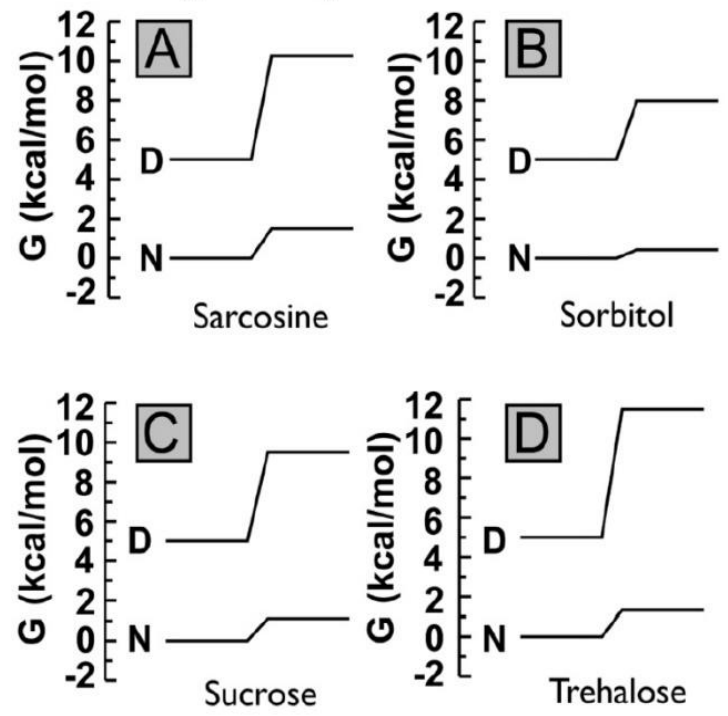

Figure 1.6. Stabilization of the native protein fold by protective osmolytes.

The left part of the line is the protein in $0 \mathrm{M}$ osmolyte, and the right side is after transfer to $1 \mathrm{M}$ osmolyte. While there is an overall increase in free energy regardless of state, the increase is higher for denatured protein, so the native state is favored. (Adapted from Auton et al., 2011) 
The peptide backbone is better solvated in pure water than a solution of osmolytes and is said to be osmophobic, which drives the preferential exclusion (Figure 1.7).

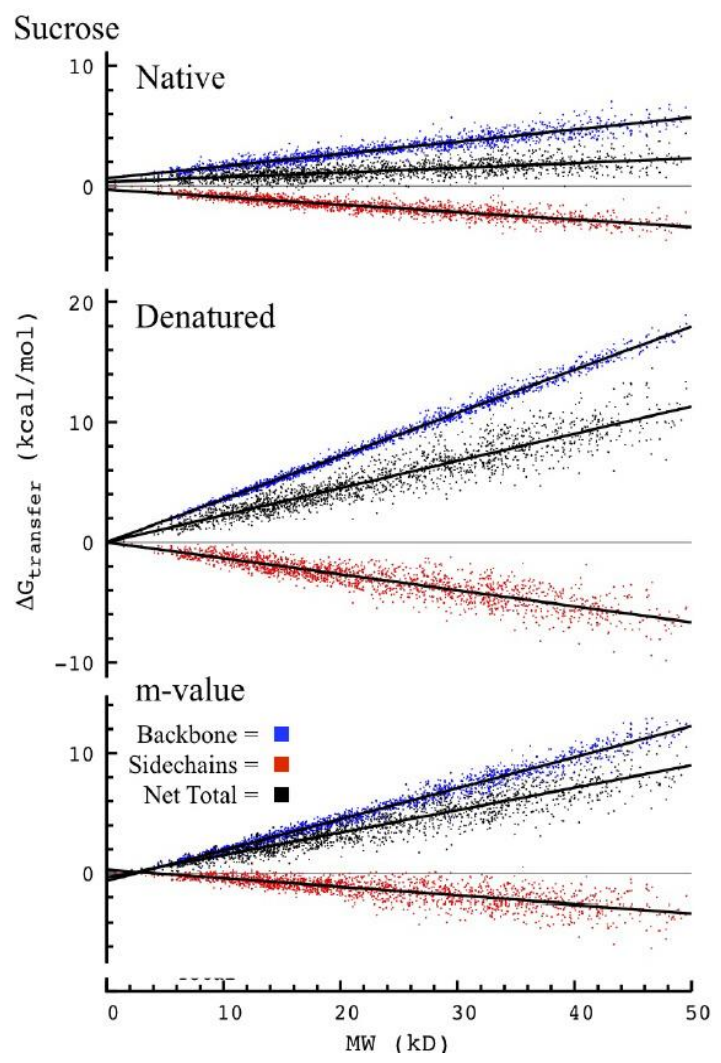

Figure 1.7. Contributions to transfer free energy of the protein backbone and side chains. Proteins of various molecular weights were transferred to 1 $M$ sucrose in either the native or denatured states. In both cases the largest contribution to the total transfer free energy was from the peptide backbone. (Adapted from Auton et al., 2011)

Current models for osmolyte stabilization of the native fold describe the mechanism through water or osmolyte interactions with the polar amide or carbonyl groups in the protein backbone. Water molecules, with their high polarity, are more likely to interact with the polar backbone groups than large, organic protective osmolytes 
(Street et al., 2006). The preferential exclusion of the osmolytes is effectively the protein doing work, which raises the free energy of the system. Since more polar backbone groups are exposed in the denatured state, this would necessarily lead to a larger free energy increase versus the native fold (Street et al., 2006).

Since backbone burial is preferred, the addition of osmolytes favors protein conformations with the lowest solvent accessible surface area, which has been used to probe conformational exchange events in the Ton box of BtuB upon substrate binding using site directed spin labeling electron paramagnetic resonance (SDSL-EPR). The spin labels used for SDSL-EPR have small solvent accessible surface areas that are not changed by sucrose addition, so rotameric states of the spin label are not altered. This allows changes in the spectra to be connected for conformational exchange events. (Figure 1.8) (Freed et al., 2010; Fanucci et al., 2003; Flores-Jimenez et al., 2010; Lopez et al., 2009).

Protective osmolytes are used extensively in crystallography as protein stabilizers and precipitants (Harries, 2008). While a series of crystal structures in different conformational states can provide an outline of a transport mechanism, the presence of protective osmolytes, detergents, and the formation of the crystal lattice necessarily restrict conformations that can be captured in crystal structures. The focus of this work is to describe conformational exchange events in the glutamate transporter homologue, $\mathrm{Glt}_{\mathrm{ph}}$, that have not been described by the crystal structures. Chapter 2 discusses the

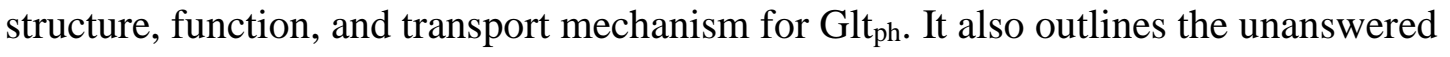
questions in the field and experimental work that is presented in Chapter 4. 

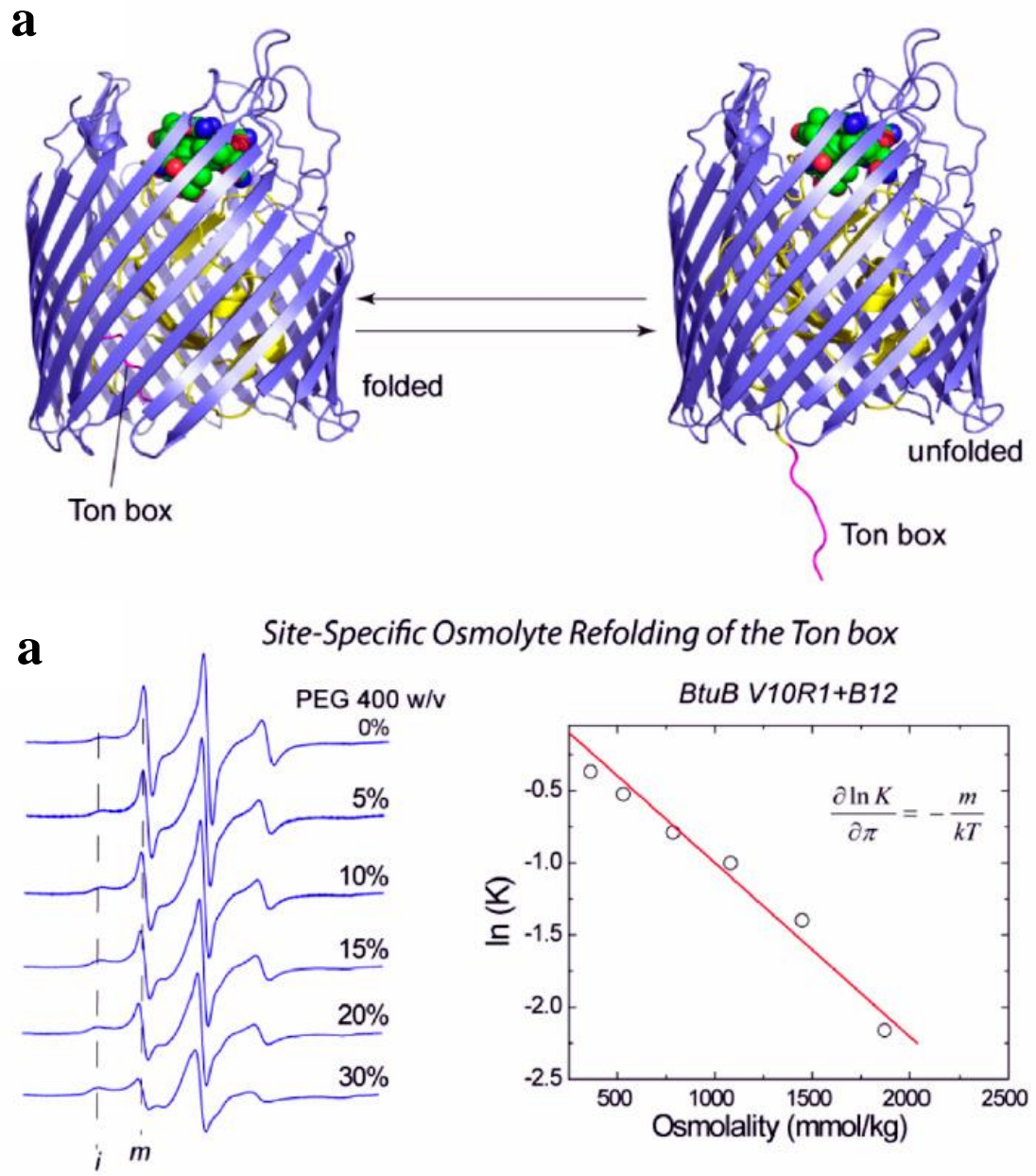

Figure 1.8. Probing conformational exchange events in the Ton box of BtuB using the protective osmolyte polyethylene glycol (PEG). a) The folded and unfolded states of the Ton box. b) CW-EPR spectral broadening with PEG titration shows the modulation of conformational equilibria with different solvent accessible surface areas. (Adapted from Cafiso, 2014) 
Chapter 2

The Solute Carrier Superfamily and Glt $t_{\mathrm{ph}}$ 


\subsection{The Solute Carrier (SLC) Superfamily}

The SLC superfamily is the second largest group of membrane proteins (second to the G-protein coupled receptors), and the largest group of human transporters (Figure 2.1) (Hediger et al., 2004; Hediger et al., 2013). It is broken down into 52 families based on topology and sequence similarity with members of each family sharing at least $20 \%$ sequence identity (Hediger et al., 2004). Most members are secondary active transporters that typically use the energy stored in an ion gradient to move substrate against its concentration gradient.

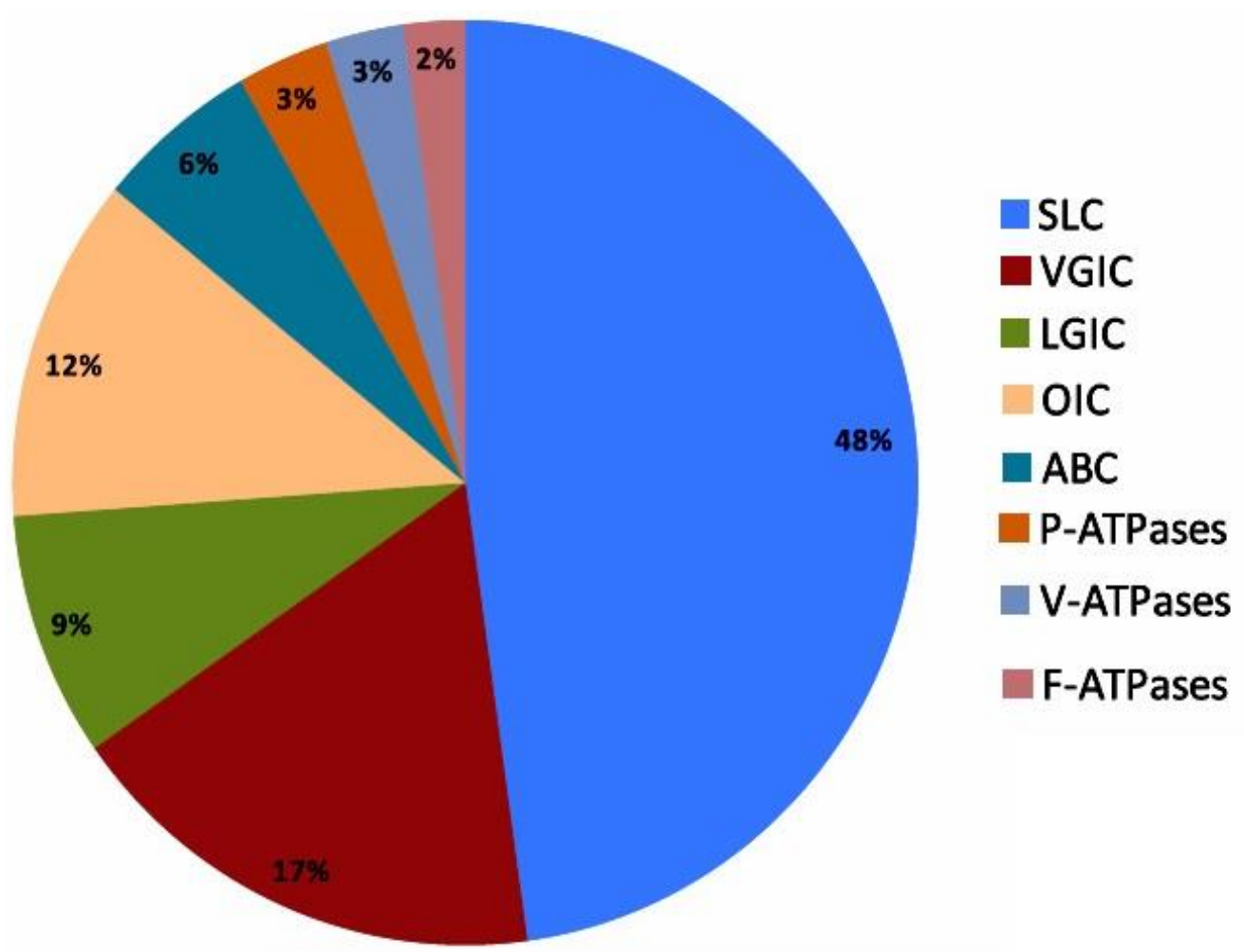

Figure 2.1. Genes encoding human transporter proteins. SLC = solute carrier; VGIC $=$ voltage gated ion channels; LGIC = ligand gated ion channels; $\mathrm{OIC}=$ other ion channels; $\mathrm{ABC}=\mathrm{ABC}$ transporters; $\mathrm{P}$-ATPases $=$ P-type ATPases; VATPases = V-type ATPases; F-ATPases = F-type ATPases. (Adapted from Hediger et al., 2013)

SLCs are essential for maintaining homeostasis as they transport a wide variety of substrates including amino acids, ions, and vitamins, among others. Defects in these 
transporters have been implicated in a wide variety of diseases such as Alzheimer's disease, epilepsy, and schizophrenia (Hediger et al., 2013; Lin et al., 2015).

Most SLCs share several common folds, despite a large sequence diversity among families. These folds all share inverted topology repeats and broken helices that necessarily lead to the alternating access transport model (Saier, 2000; Jardetzky, 1966; Forrest et al., 2008; Kanner and Zomot, 2008; Krishnamurthy et al., 2009). Specific details about the alternating access model have been described using the crystal structures from SLC members (Figure 2.2) (Hediger et al., 2013).

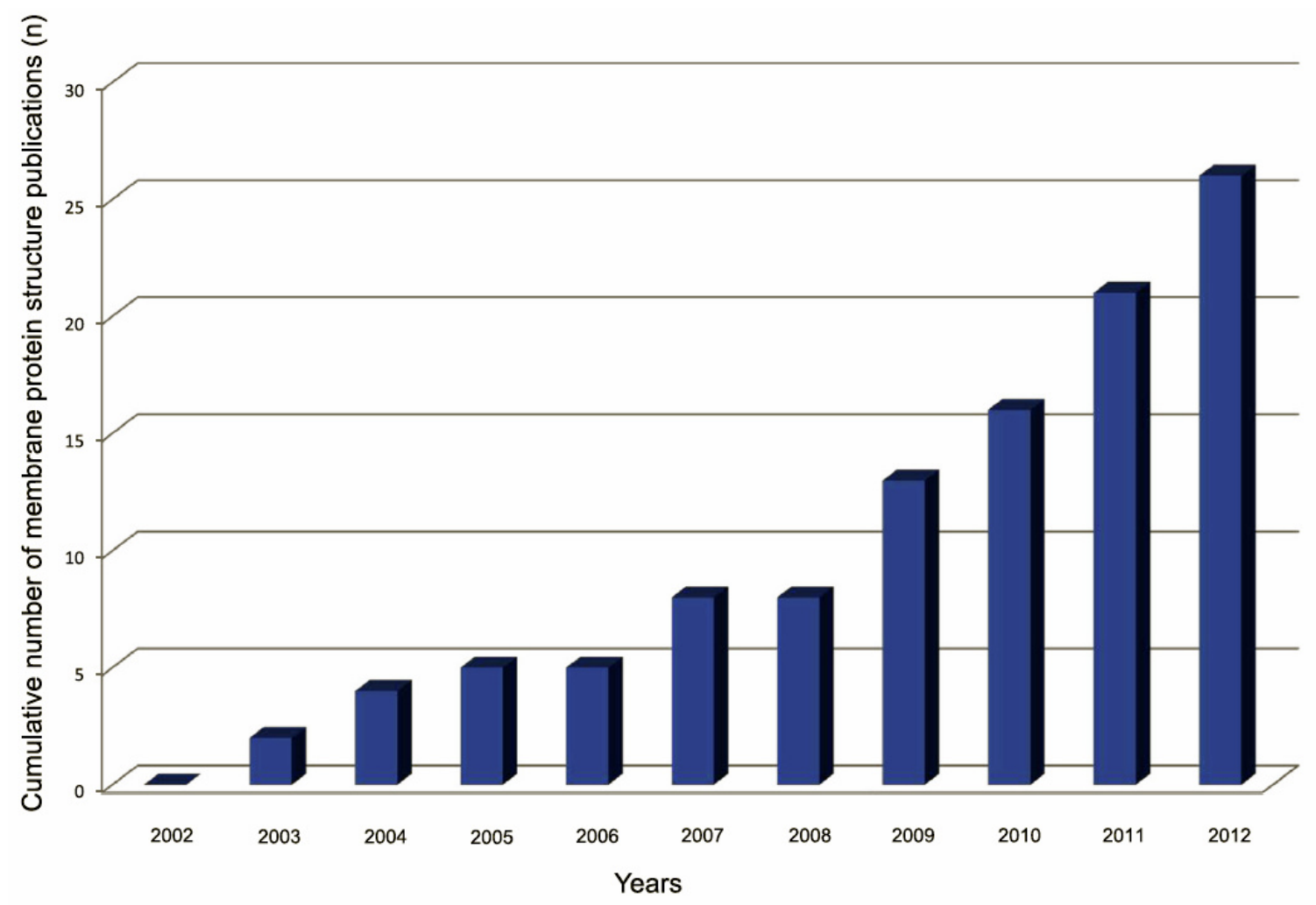

Figure 2.2. Cumulative number of crystal structures of SLC family members. (Hediger et al., 2013) 
The SLC1 family contains five glutamate transporters which share $45-55 \%$ sequence identity with the other glutamate transporters and two neutral amino acid transporters that share $57 \%$ sequence identity with each other (Table 2.1). The glutamate transporters are responsible for tightly controlling glutamate concentrations in the central and peripheral nervous systems to stop signaling events and prevent excitotoxicity and its associated diseases (Table 2.1) (Kanai et al., 2013).

Table 2.1 The SLC1 Transporter Family (Adapted from Kanai et al., 2004).

\begin{tabular}{|c|c|c|c|c|c|c|}
\hline $\begin{array}{l}\text { Human } \\
\text { gene } \\
\text { name }\end{array}$ & $\begin{array}{l}\text { Protein } \\
\text { name }\end{array}$ & Aliases & $\begin{array}{l}\text { Predominant } \\
\text { substrates }\end{array}$ & $\begin{array}{l}\text { Transport } \\
\text { type/ } \\
\text { coupling } \\
\text { ions }\end{array}$ & $\begin{array}{l}\text { Tissue } \\
\text { distribution and } \\
\text { cellular/ } \\
\text { subcellular } \\
\text { expression }\end{array}$ & Link to disease \\
\hline SLC1A1 & $\begin{array}{l}\text { EAAC1, } \\
\text { EAAT3 }\end{array}$ & $\begin{array}{l}\text { System } \\
\mathrm{X}^{-}{ }_{\mathrm{AG}}\end{array}$ & L-Glu, D/L-Asp & $\begin{array}{l}\mathrm{C} / \mathrm{Na}^{+}, \mathrm{H}^{+} \\
\text {and } \mathrm{K}^{+}\end{array}$ & $\begin{array}{l}\text { Brain (neurons), } \\
\text { intestine, kidney, } \\
\text { liver, heart, } \\
\text { placenta }\end{array}$ & $\begin{array}{l}\text { Huntington's disease, Epilepsy, } \\
\text { Ischemia, Alzheimer's disease, } \\
\text { Niemann-Pick disease, } \\
\text { Obsessive-compulsive } \\
\text { disorder }\end{array}$ \\
\hline SLC1A2 & $\begin{array}{l}\text { GLT-1, } \\
\text { EAAT2 }\end{array}$ & $\begin{array}{l}\text { System } \\
\mathrm{X}^{-}{ }_{\mathrm{AG}}\end{array}$ & L-Glu, D/L-Asp & $\begin{array}{l}\mathrm{C} / \mathrm{Na}^{+}, \mathrm{H}^{+} \\
\text {and } \mathrm{K}^{+}\end{array}$ & $\begin{array}{l}\text { Brain (astrocytes, } \\
\text { Bergmann glia, } \\
\text { neurons), liver, } \\
\text { pancreas }\end{array}$ & $\begin{array}{l}\text { Amyotrophic lateral sclerosis, } \\
\text { Alzheimer's disease, } \\
\text { Huntington's disease, Epilepsy, } \\
\text { Ischemia, Schizophrenia }\end{array}$ \\
\hline SLC1A3 & $\begin{array}{l}\text { GLAST, } \\
\text { EAAT1 }\end{array}$ & $\begin{array}{l}\text { System } \\
\mathrm{X}^{-} \text {AG }\end{array}$ & L-Glu, D/L-Asp & $\begin{array}{l}\mathrm{C} / \mathrm{Na}^{+}, \mathrm{H}^{+} \\
\text {and } \mathrm{K}^{+}\end{array}$ & $\begin{array}{l}\text { Brain (astrocytes, } \\
\text { Bergmann glia), } \\
\text { heart, skeletal } \\
\text { muscle, placenta }\end{array}$ & $\begin{array}{l}\text { Alzheimer's disease, } \\
\text { Huntington's disease, Epilepsy, } \\
\text { Cerebellar ataxia type } 7 \text {, } \\
\text { Schizophrenia }\end{array}$ \\
\hline SLC1A4 & $\begin{array}{l}\text { ASCT1, } \\
\text { SATT }\end{array}$ & $\begin{array}{l}\text { System } \\
\text { ASC }\end{array}$ & $\begin{array}{l}\text { L-Ala, L-Ser, L- } \\
\text { Cys, L-Thr }\end{array}$ & $\begin{array}{l}\mathrm{C} / \mathrm{Na}^{+}, \mathrm{E} / \\
\text { amino } \\
\text { acids }\end{array}$ & Widespread & \\
\hline SLC1A5 & $\begin{array}{l}\text { ASCT2, } \\
\text { AAAT }\end{array}$ & $\begin{array}{l}\text { System } \\
\text { ASC }\end{array}$ & $\begin{array}{l}\text { L-Ala, L-Ser, L- } \\
\text { Cys, L-Thr, L- } \\
\text { Gln, L-Asn }\end{array}$ & $\begin{array}{l}\mathrm{C} / \mathrm{Na}^{+}, \mathrm{E} / \\
\text { amino } \\
\text { acids }\end{array}$ & $\begin{array}{l}\text { Lung, skeletal } \\
\text { muscle, large } \\
\text { intestine, kidney, } \\
\text { testis, adipose } \\
\text { tissue }\end{array}$ & \\
\hline SLC1A6 & EAAT4 & $\begin{array}{l}\text { System } \\
\mathrm{X}^{-} \mathrm{AG}\end{array}$ & L-Glu, D/L-Asp & $\begin{array}{l}\mathrm{C} / \mathrm{Na}^{+}, \mathrm{H}^{+} \\
\text {and } \mathrm{K}^{+}\end{array}$ & $\begin{array}{l}\text { Cerebellum } \\
\text { (Purkinje cells) }\end{array}$ & Spinocerebellar ataxia type 5 \\
\hline SLC1A7 & EAAT5 & $\begin{array}{l}\text { System } \\
\mathrm{X}^{-}\end{array}$ & L-Glu, D/L-Asp & $\begin{array}{l}\mathrm{C} / \mathrm{Na}^{+}, \mathrm{H}^{+} \\
\text {and } \mathrm{K}^{+}\end{array}$ & $\begin{array}{l}\text { Retina (rod } \\
\text { photoreceptors } \\
\text { and bipolar cells }\end{array}$ & \\
\hline
\end{tabular}

Three $\mathrm{Na}^{+}$ions and one $\mathrm{H}^{+}$are coupled to glutamate reuptake with one $\mathrm{K}^{+}$ion countertransported to complete the cycle. This concentrates glutamate in neurons to 
approximately $10 \mathrm{mM}$, while maintaining synaptic concentrations of approximately $1 \mu \mathrm{M}$ (Zerangue et al., 1996). Transport is electropositive with a thermodynamically uncoupled, substrate gated $\mathrm{Cl}^{-}$conductance that is thought to partially dissipate the membrane potential, maintaining transport rates (Arriza et al., 1997; Fairman et al., 1995; Wadiche et al., 1995a).

While sharing an overall transport mechanism and substrate binding stoichiometry, glutamate transporters differ in their distribution and overall effect on glutamate concentrations. Synaptic glutamate concentrations are primarily controlled by SLC1A2 (GLT-1), which is responsible for approximately 95\% of glutamate reuptake (Rothstein et al., 1995). After a glutamatergic signaling event, GLT-1 rapidly removes glutamate, concentrating it in glial cells, where it can be converted to glutamine. Since glutamine is not a neurotransmitter, it can be released into the synaptic space for transport back to the neurons (Figure 2.3) (Kanai et al., 2013). Previous gene knockout studied in mice have shown that GLT-1 and SLC1A3 (GLAST) are both necessary to maintain the proper glutamate concentration, while SLCA1 (EAAC1) is not required (Rothstein et al., 1996).

\subsection{The glutamate transporter homologue Gitph}

Many functional studies on glutamate transporters have been guided by recent crystal structures of the sodium dependent aspartate transporter $\mathrm{Glt}_{\mathrm{ph}}$. Glt $\mathrm{ph}_{\mathrm{ph}}$ shares approximately $30 \%$ sequence identity with the glutamate transporters and $37 \%$ sequence identity with GLT-1, making it an excellent structural and functional model (Figure 2.4) (Yernool et al., 2004). Similar to glutamate transporters, Gltph binds three $\mathrm{Na}^{+}$ions with 


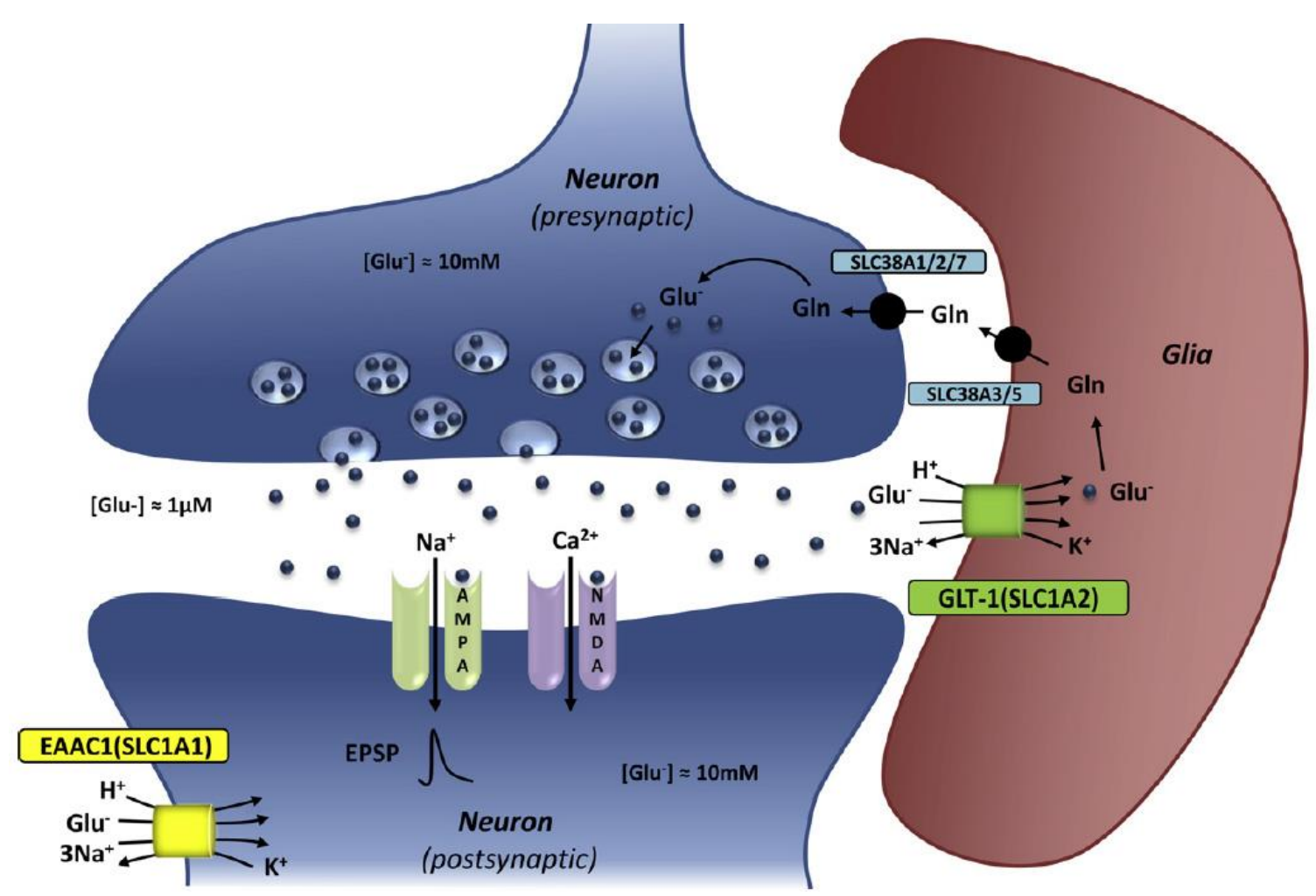

Figure 2.3. Overview of glutamatergic signaling. Glutamate reuptake is primarily controlled by GLT-1, which concentrates glutamate in glial cells for conversion to glutamine, which is not a neurotransmitter and can be released back into the synaptic space for transport back to neurons (Kanai et al., 2013).

substrate; however, in contrast, transport is not $\mathrm{H}^{+}$or $\mathrm{K}^{+}$dependent (Ryan et al., 2009).

The Glt $\mathrm{ph}_{\mathrm{p}}$ crystal structure shows a bowl-shaped homotrimer with an aqueous basin lined with hydrophilic residues that directly allows access to the substrate binding sites (Figure 2.5). There are eight TM helices, two reentrant broken helices, and a proline rich linker that coordinates the movement of aspartate through the membrane. Helices 2, 4, and 5 form a trimerization domain that mediates intersubunit contacts. Helices 3, 6, 7 and 8 and the two broken helices (HP1 and HP2) form the transport domain, and the two domains are linked through the 3-4 loop (Yernool et al., 2004). 


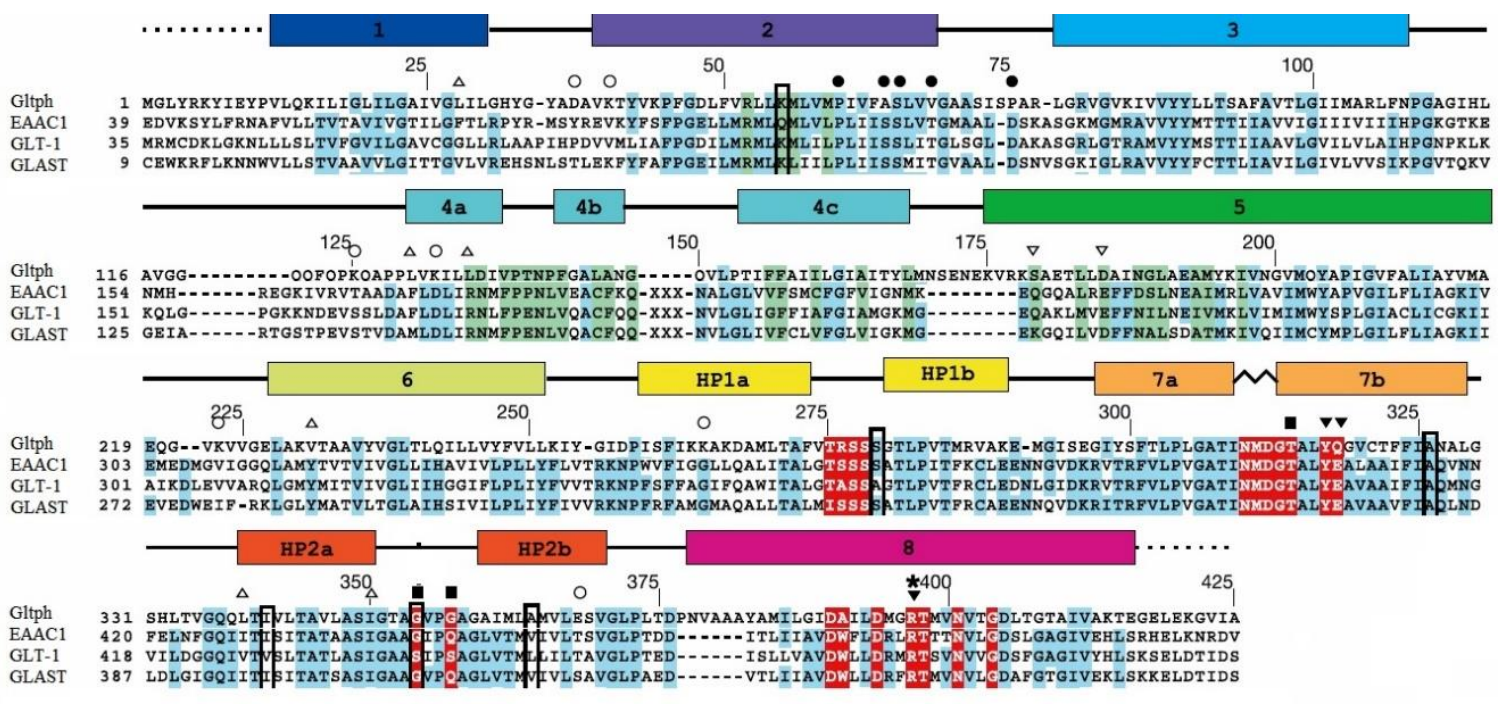

Figure 2.4. Sequence homology of Gltph with three synaptic glutamate

transporters. Labels for TM helices are above the sequences. Areas of high sequence homology are highlighted in blue; intermonomer interfacial resides are highlighted in green; residues implicated in substrate binding and transport are highlighted in red. Black squares indicate residues that are important for sodium binding; inverted triangles indicated residues in glutamate transporters that are important for potassium transport; filled circles are residues that are important for chloride conductance. Open symbols denote mutations. (Adapted from Yernool et al., 2004)

\subsubsection{The structure of Glt $_{\mathrm{ph}}$}

The trimerization domain (Figure 2.5, tan) contains all of the intersubunit contacts. TM 4 is a broken helix that is divided into three sections (4a, $4 \mathrm{~b}$, and $4 \mathrm{c})$ and participates in all interactions. TM helix $4 \mathrm{a}$ interacts with neighboring TM 2; TM 4b in each protomer interact; TM 4c interacts with neighboring TM 5 (Yernool et al., 2004). Cysteine crosslinks engineered between these helices did not affect transport activity, showing that movements in the trimerization domain are not necessary for transport and that it is most likely a rigid scaffold (Groeneveld and Slotboom, 2007). 

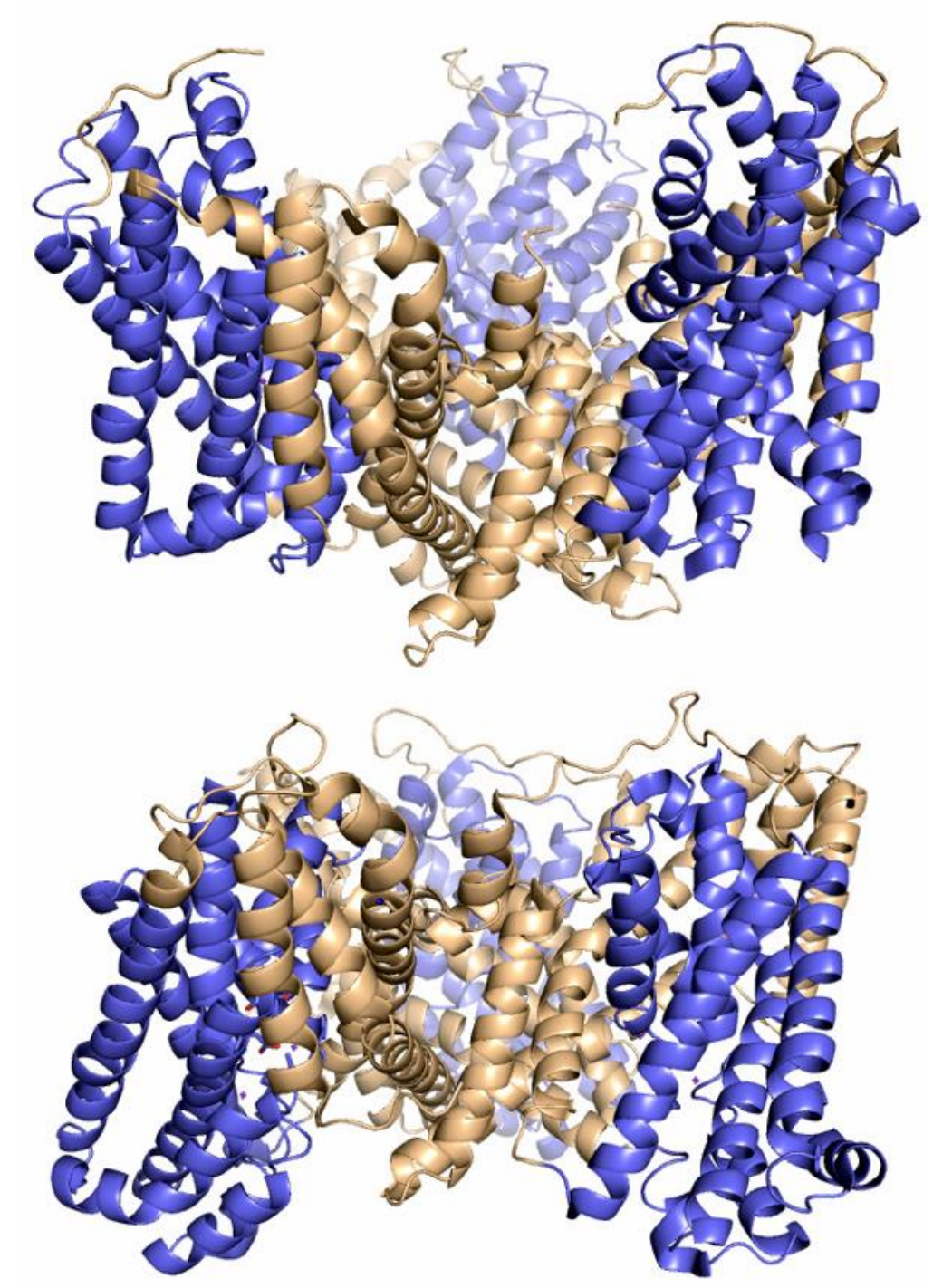

Figure 2.5. Crystal structures of the outward (PDB ID: 2NWX) and inward (PDB ID: 3KBC) facing conformations of $\mathbf{G l t}_{\mathrm{ph}}$. The trimerization domain (TMs 2, 4, 5) are in tan and the transport domain (TMs 3, 6, 7, 8, HP1, HP2) are shown in blue.

The transport domain contains all of the substrate binding sites and the structural features necessary for protomer reorientation. TMs 3 and 6 provide scaffolding for the rest of the transport domain, and the loops that connect these helices to the trimerization domain undergo conformational changes that allow movement to the inward facing 
conformation (Reyes et al., 2009). The 3-4 loop, which connects the transport domain to the trimerization domain, is a 19 residue-long loop that is necessary for transport. It has been shown to undergo substrate induced conformational changes, and cleaving the loop greatly decreases transport (Compton et al., 2010). However, its specific role in substrate transport is not well understood. TMs 7 and 8 and HP1 and HP2 form the aspartate and sodium binding sites. The aspartate binding site is formed primarily by the tips of HP1 and HP2 with residues in TM 7 (T314) and TM 8 (D394 and R397) also coordinating substrate through interactions with substrate carboxyl groups (Boudker et al., 2007). One sodium binding site is formed by the unwound NMGDT motif of TM 7 and residue D405 in TM 8; the second sodium binding site is formed by residues in TM 7 (T308) and HP2 (S349 and T352). The location of the third sodium binding site is debated but has been proposed by simulations to be between TMs 3 and 7 (Boudker et al., 2007; Verndon et al, 2014; Jiang and Amara, 2011; Larsson et al., 2010). HP1 and HP2 have been proposed to be intracellular and extracellular gates (Figure 2.6). A crystal structure of Glt $\mathrm{ph}_{\text {bound to }}$ the transport inhibitor DL-threo- $\beta$-benzyloxyaspartate (DL-TBOA) showed HP2 propped in an open conformation by the benzyl group on TBOA, providing evidence that it is the extracellular gate (Boudker et al., 2007). Subsequent CW-EPR studies showed that HP2 undergoes opposite movements when sodium and aspartate are bound, with sodium opening HP2 and aspartate closing it (Focke et al., 2011). Simulations have shown that movements of both HP1 and HP2 are necessary for substrate release intracellularly. HP2 moved approximately $4 \AA$ away from the substrate binding site, providing space for the aspartate to interact with the side chain of R276 and subsequently be released (Figure 
2.6) (Zomot and Bahar, 2013); however, gate-like movements for HP1 are proposed to be very small and would be difficult to experimentally observe.

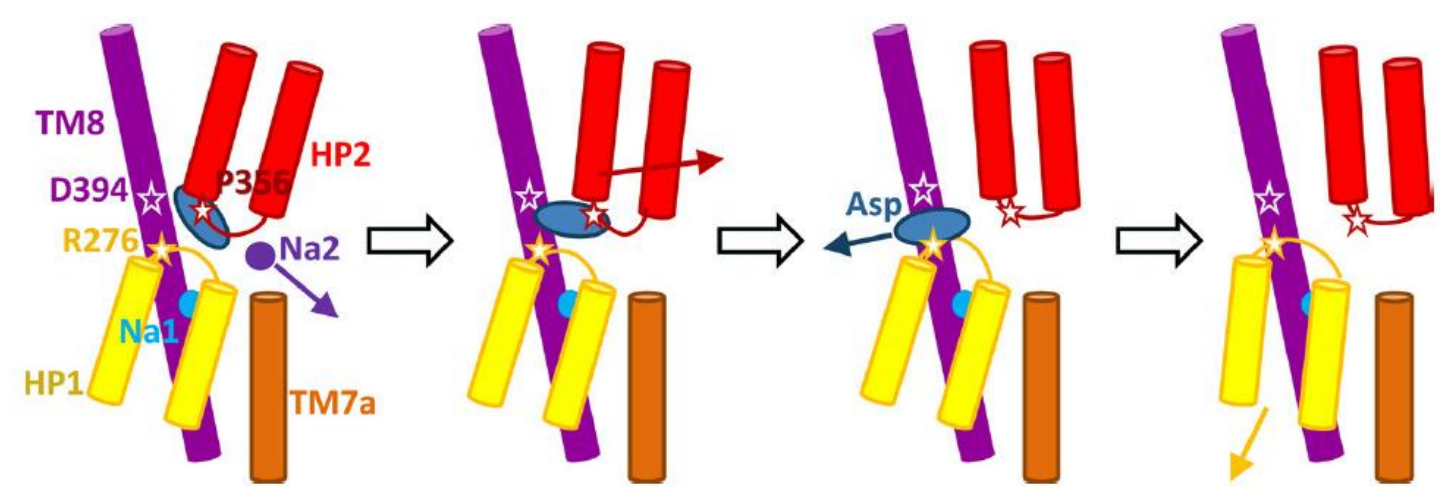

Figure 2.6. Gate movements of HP1 and HP2 in the inward facing

conformation. HP2 (red) moves away from the aspartate binding site, followed by an electrostatic interaction between the bound aspartate and R276 on HP1 (yellow). Small scale movements of HP1 would allow aspartate release (Zomot and Bahar, 2013).

\subsubsection{The elevator model}

Similar to other secondary active transporters, Glt $\mathrm{ph}$ transports substrate by the alternating access mechanism; however, a crystal structure of the inward facing conformation described a more complicated mechanism. The transport domains, while structurally similar to the outward facing state, had moved approximately $15 \AA$ across the membrane and rotated approximately $30^{\circ}$, giving the substrate binding sites intracellular access (Figure 2.7) (Reyes et al., 2009). Now termed the 'elevator model,' these rigid body movements have recently been experimentally observed by high speed atomic force microscopy (Ruan et al., 2017). Each protomer can undergo these transitions independently (Erkins et al., 2013). 


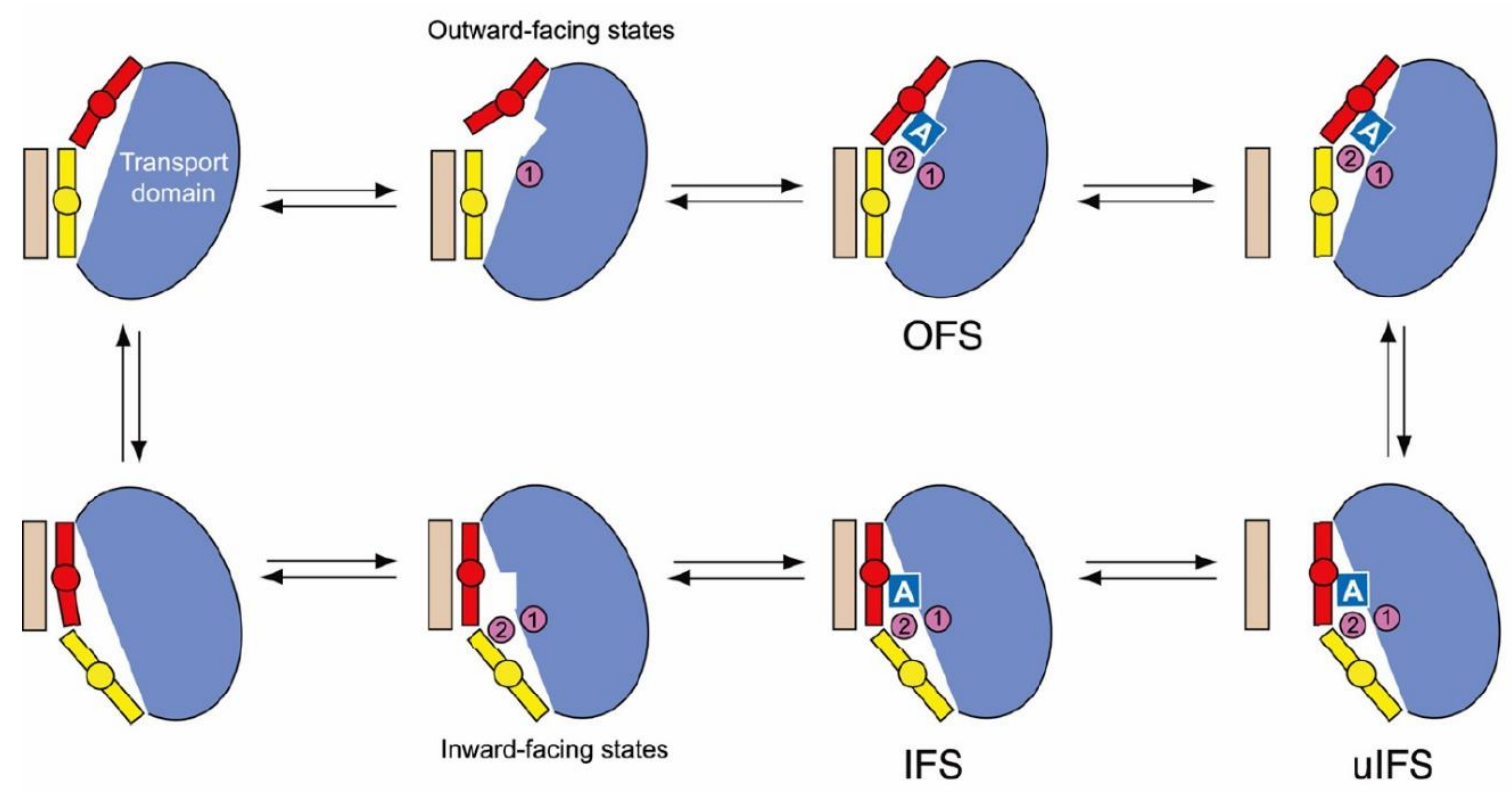

Figure 2.7. The alternating access/elevator model of transport. Upon at least one sodium binding, HP2 (red) opens, giving access to the substrate binding site. Aspartate binding closes HP2, and an unlocking step from the trimerization domain (tan) allows transport domain (blue) movement through the bilayer, giving the substrate binding site intracellular access. Small scale movements of both HP2 and HP1 (yellow) are necessary to release substrate. The mechanism of empty transporter reorientation is currently unknown (Adapted from LeVine et al., 2016).

\subsection{3 $\mathrm{Na}^{+}$and aspartate coupling}

The coupling of sodium and aspartate binding is complex, involving at least two mechanisms. CW-EPR distance measurements showed a closed aspartate binding site until at least one $\mathrm{Na}^{+}$was bound, which opened HP2; however, binding measurements in detergent showed that aspartate was coupled to at least two $\mathrm{Na}^{+}$(Focke et al., 2011; Boudker et al., 2007; Hanelt et al., 2015). The low affinity $\mathrm{Na}^{+}$binding $\left(\mathrm{K}_{\mathrm{D}}: 120 \mathrm{mM}\right)$ in the absence of aspartate increased in the presence of aspartate $\left(\mathrm{K}_{\mathrm{D}}: 25 \mathrm{mM}\right)$, and the aspartate binding affinity was drastically affected by $\mathrm{Na}^{+}$concentrations $\left(\mathrm{K}_{\mathrm{D}}\right.$ at $10 \mathrm{mM}$ $\mathrm{Na}^{+}: 4 \mu \mathrm{M} ; \mathrm{K}_{\mathrm{D}}$ at $200 \mathrm{mM} \mathrm{Na}^{+}: 2 \mathrm{nM}$ ) (Hanelt et al., 2015). This would be consistent with an induced fit binding model in which a loose association to the substrate binding 
site allows small scale conformational changes that promote higher-affinity binding.

(Ewers et al., 2013).

\subsubsection{Thermodynamics and kinetics of conformational exchange}

Free energies of distinct conformations and the kinetics of conformational exchange in $\mathrm{Glt}_{\mathrm{ph}}$ have been probed using a variety of methods. Conformational populations calculated through double electron-electron resonance (DEER) distance distributions and single molecule fluorescence resonance energy transfer (sm-FRET) experiments have shown that the $\Delta \Delta \mathrm{G}$ of the outward and inward conformations is equal (Figure 2.8) (Akyuz et al., 2013; Georgieva et al., 2013; Hanelt et al., 2013; Andersen and Koeppe, 2007). DEER experiments showed the addition of $\mathrm{Na}^{+}$and $\mathrm{Na}^{+}$and aspartate stabilized the outward facing conformation, although site specific stabilization of the inward facing conformation has been observed (Akyuz et al.,2013; Georgieva et al., 2013) (Figure A.1). These experiments assumed that the outward and inward facing conformations are in equilibrium. In the presence of substrate, transitions between

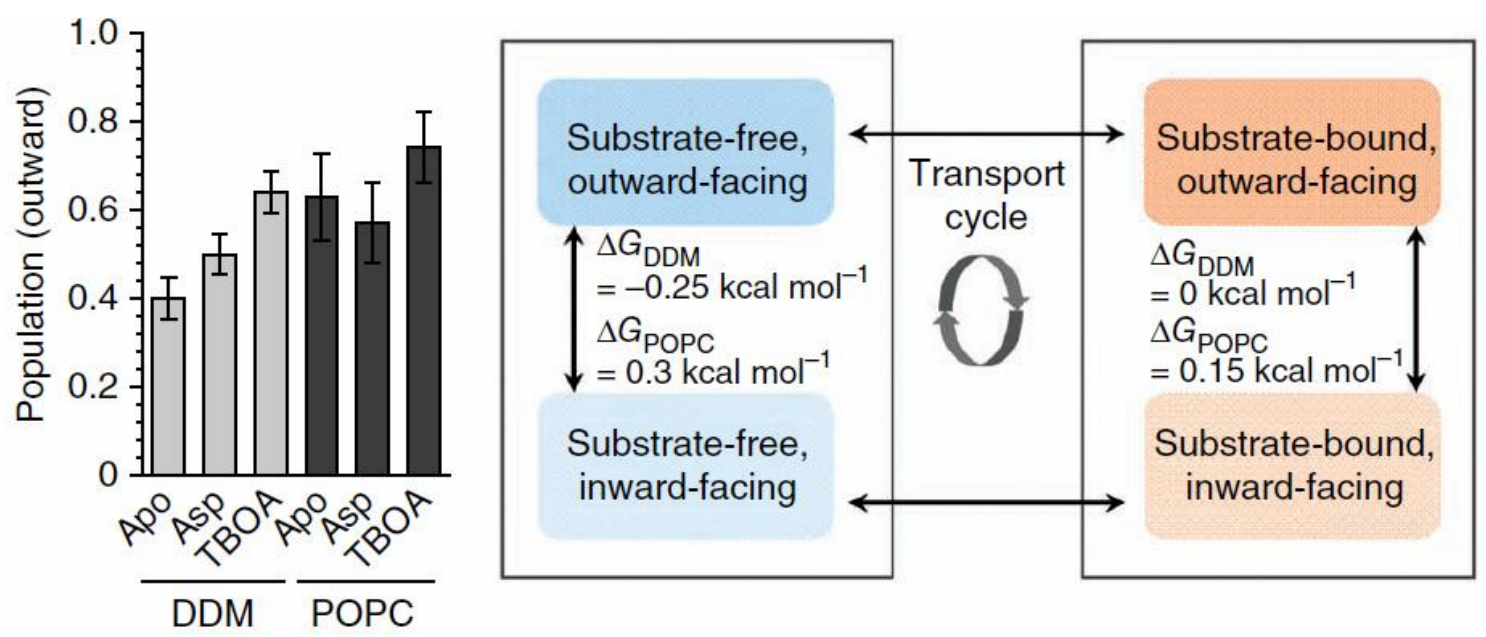

Figure 2.8. The outward and inward facing conformations have nearly equal free energies. Average populations were calculated through Gaussian fits of DEER distance distributions based on distance expectations from crystal structures of the outward (2NWX) and inward (3KBC) conformations. A model for the intermediate conformation was generated from the two previous structures (Georgieva et al., 2013). 
conformations undergo cycles of rapid movement and 'quiet' periods when no large-scale exchange is occurring. Substrate loaded transitions occur on average every 100 seconds, while substrate free transitions occur on average every two seconds, suggesting that there is a rate limiting step in the substrate loaded transporter due to a structural difference during interconversion (Akyuz et al., 2013). Transport domain unlocking from the trimerization domain has been suggested as the rate limiting step, and a gain-of-function double mutant (R276S/M395R) allowed this conformation to be captured crystallographically (Akyuz et al., 2015).

\subsubsection{Intermediate conformations and influence of the lipid bilayer}

Interprotomer DEER distance distributions in the transport domain were typically broad, suggesting unresolved intermediate states (Akyuz et al., 2013; Georgrieva et al, 2013). Conformational heterogeneity can also be studied by measuring changes in protein heat capacity, which can be related to specific events during substrate binding (Prabhu and Sharp, 2005). A study calculating heat capacity changes in the outward and inward facing conformations showed that there were large negative heat capacity changes upon $\mathrm{Na}^{+}$and aspartate binding, which could be due to either the burial of a large hydrophobic surface or the collapse of conformational heterogeneity into one state upon substrate binding. However, the heat capacity change was twice as large when substrate bound the inward facing state, which contradicted the similarity of the binding sites in the crystal structures. (Reyes et al., 2013).

The binding studies and some DEER measurements used detergent solubilized $\mathrm{Glt}_{\mathrm{ph}}$. While some measurements in a lipid bilayer environment have been consistent with measurements in detergent, others have shown conformational modulation. Substrate free 
transport domain interconversions were slowed by a factor of two in 3:1 E. coli polar lipid:POPC liposomes (Akyuz et al., 2015). Conformational populations were shifted between detergent solubilized $\mathrm{Glt}_{\mathrm{ph}}$ and $\mathrm{Glt}_{\mathrm{ph}}$ reconstituted in a POPC bilayer, with the bilayer sometimes increasing conformational heterogeneity or stabilizing a specific state, decreasing conformational heterogeneity (Figure 2.9) (Akyuz et al., 2013; Georgieva et al., 2013). The identity of the lipid in the bilayer also affects transport rates, with POPE supporting the fastest transport and POPC the slowest transport (McIlwain et al., 2015). This suggests a complex underlying transport mechanism that cannot be fully explained by the crystal structures.

I294R1
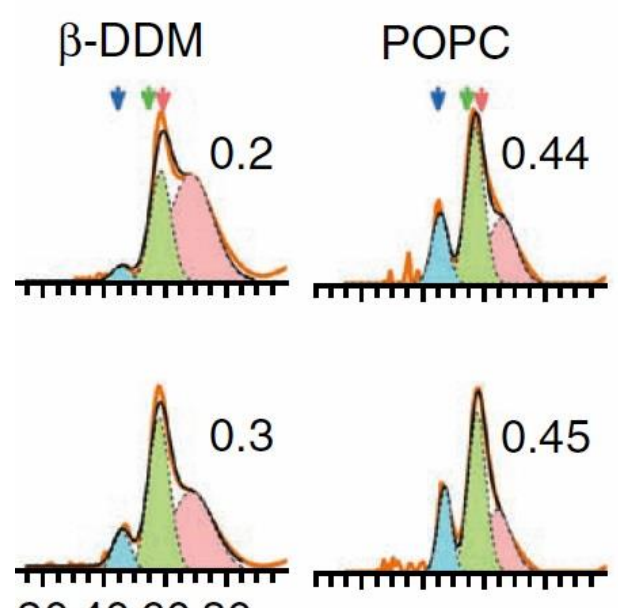

20406080
E296R1
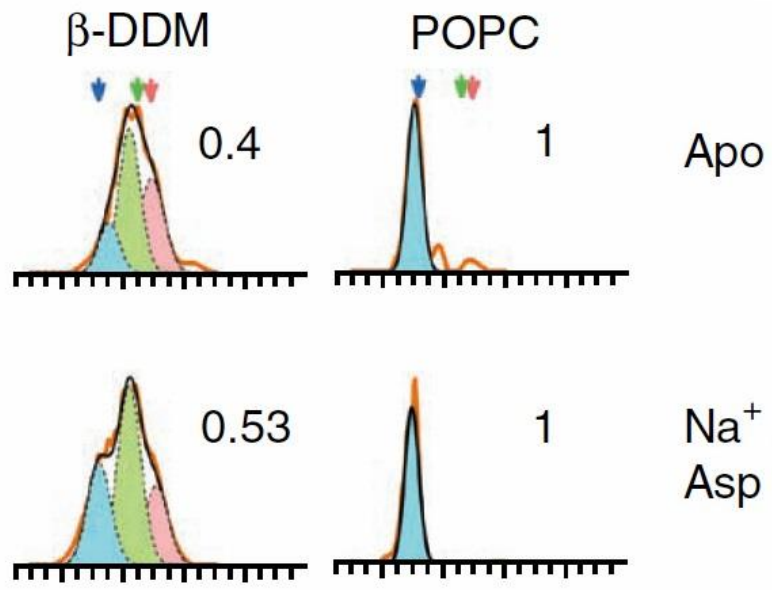

$\mathrm{Na}^{+}$and

Asp

Figure 2.9. Transport domain conformational heterogeneity in detergent micelles and POPC bilayers. Broad distributions in both environments suggest conformational heterogeneity; however, the lipid bilayer favors different conformations than detergent. The colored Gaussians represent MMM rotamer library fits based on the outward (blue), inward (pink), and intermediate (green) models (Adapted from Georgieva et al., 2013). 
This work aims to provide more details on the transport mechanism by studying Glt $_{\mathrm{ph}}$ in a lipid bilayer under conditions that support transport. The role of HP1 in intracellular gating remains unclear based on the crystal structures, which always show it coordinating substrate and interacting with TM 8. Conformational changes in the 3-4 loop that mediate substrate-loaded transport domain reorientation are also not detailed as the loop is the site of crystal lattice contacts. Furthermore, there are higher energy intermediate states that must mediate transport that cannot be captured crystallographically. These conformational exchange events were visualized using electron paramagnetic resonance, which is well suited for experiments in bilayers as the technique is not limited by the size of the system. Applications, interpretation, and theory of continuous wave (CW) and pulsed EPR techniques is discussed in Chapter 3. 
Chapter 3

EPR Theory and Applications 


\subsection{EPR Theory}

\subsubsection{The Resonance Condition}

An electron has inherent spin angular momentum and, therefore, a magnetic moment

$$
\mu=-g \beta_{e} \mathrm{~S}
$$

where $\mathrm{g}$ is the $\mathrm{g}$-factor and $\beta_{e}$ is the Bohr magneton

$$
\beta_{e}=\frac{e \hbar}{2 m_{e}}
$$

with e as the charge of an electron, $\hbar=\frac{h}{2 \pi}$, and $m_{e}$ as the mass of an electron.

Contributions from the orbital angular momentum (L) are neglected because the ground state energies of the orbitals $p_{x}, p_{y}, p_{z}$ are not degenerate. The energy of an electron is described by the Hamiltonian $(\hat{H})$. Energy levels are degenerate until a magnetic field is applied. With a static field applied in the z-direction,

$$
\hat{\mathrm{H}}_{e}=-\mu B_{0}=g \beta_{e} S_{z} B_{0}
$$

The solutions for the spin angular momentum quantum numbers $\left(+\frac{1}{2},-\frac{1}{2}\right)$ are substituted for $S_{z}$ to give the energy

$$
E= \pm \frac{1}{2} g \beta_{e} B_{0}
$$

and the difference between the two energy levels is dependent on the strength of the static field. Energy absorption occurs when the applied energy is equal to the difference between the two energy levels

$$
h v=\Delta E=g \beta_{e} B_{0}
$$


This condition is called resonance and occurs at the sample's Larmor frequency

$$
\omega_{0}=\gamma B_{0}
$$

where $\omega_{0}=2 \pi v$ and $\gamma=\frac{g \beta_{e}}{h}$.

\subsubsection{The g-tensor}

The g-factor, which has a value of 2.00232 for a free electron, describes the local field experienced by an electron and is influenced by both the static field and spin-orbit coupling due to the fields from other electrons. Both the static field and electron spin are dependent upon orientation; therefore, the g-factor is a second rank tensor that is represented by the diagonal of the tensor matrix so it is in the correct coordinate system, $g_{x x}, g_{y y}$, and $g_{z z}$. Since the g-factor is a measure of polarizability, it is considered a splitting factor that can describe the local environment of the electron.

\subsubsection{The A-tensor}

The A-tensor is also second rank and represented by the diagonal for the molecular frame, $A_{x x}, A_{y y}, A_{z z}$. It describes the hyperfine interaction, which is the interaction of an electron magnetic moment with a nuclear magnetic moment through the dipolar interaction and the Fermi contact interaction. The dipolar interaction is the through-space coupling of two magnetic moments and is dependent on distance and orientation. The Fermi contact interaction is the direct interaction of the nuclear and electron magnetic moments, which has a finite probability for $s$ orbital electrons. For a nitroxide, the lone electron most often occupies the $2 \mathrm{p}_{z}$ orbital, so the Fermi contact interaction is neglected due to the node at the nucleus. 
The Hamiltonian also includes electron and nuclear spin (I)

$$
\hat{\mathrm{H}}_{A}=S A I
$$

A nitroxide with an $_{7}^{14} N$ nucleus has $2 \mathrm{I}+1$ quantum states and a nuclear spin of 1 , so an EPR spectrum is split into three peaks. The nuclear magnetic moment also interacts with the static field, and this contribution must be represented in the complete Hamiltonian.

$$
\hat{\mathrm{H}}=g \beta_{e} S_{z} B_{0}+S A I-g_{N} \beta_{N} B_{0} I
$$

\subsubsection{Relaxation}

Relaxation is the set of processes that return an excited state to thermal equilibrium. In an EPR experiment, net magnetization is detected, and the populations of the two energy levels is described by the Boltzmann distribution.

$$
\frac{n_{A}}{n_{B}}=e^{\frac{-E}{k T}}
$$

At thermal equilibrium there are more $n_{B}$ spins, so absorption can occur, and there is a net magnetization over all spins $(\mathrm{N})$

$$
M=\frac{N \gamma_{e}^{2} \hbar^{2} S}{3 k T} B_{0}
$$

The presence of the static magnetic field in the $\mathrm{z}$-direction leads to differences in relaxation rates for the $\mathrm{x}, \mathrm{y}$, and $\mathrm{z}$ components that are described by the Bloch equations,

$$
\begin{gathered}
\frac{d M_{z}}{d t}=\frac{M_{z}-M}{T_{1}} \\
\frac{d M_{x}}{d t}=-\frac{M_{x}}{T_{2}}
\end{gathered}
$$




$$
\frac{d M_{y}}{d t}=-\frac{M_{y}}{T_{2}}
$$

where $T_{1}$ is a characteristic longitudinal relaxation time and $T_{2}$ is a characteristic transverse relaxation time and applies a torque that affects the net magnetization.

$$
\frac{d M}{d t}=\gamma_{e}\left(M \times B_{0}\right)
$$

The entire Bloch equation includes the field's effect on net magnetization and relaxation.

$$
\frac{d M}{d t}=\gamma_{e}\left(M \times B_{0}\right)-\frac{k\left(M_{z}-M\right)}{T_{1}}-\frac{i M_{x}+j M_{y}}{T_{2}}
$$

The net magnetization precesses around the static field at the Larmor frequency. During an EPR experiment, a sample is irradiated with microwaves, which leads to an applied field $\mathrm{B}_{1}$ in the $\mathrm{x}$-direction. This field tips the net magnetization away from equilibrium and causes the magnetization to precess around the $\mathrm{x}$-axis as long as the applied frequency is equal to the Larmor frequency.

The rotating frame is used because vectors appear fixed inside of the rotating frame but rotate outside of the frame. The Bloch equations inside the rotating frame contain in-phase $\left(M_{x}^{\prime}\right)$ and out-of-phase $\left(M_{y}^{\prime}\right)$ magnetization and the rotation outside of the reference frame

$$
\frac{\delta M^{\prime}}{\delta t}=\gamma_{e} M^{\prime} \times\left(B_{0}^{\prime}+\frac{\omega^{\prime}}{\gamma_{e}}\right)+\gamma_{e}\left(M^{\prime} \times B^{\prime}\right)-\frac{k^{\prime}\left(M_{z}-M\right)}{T_{1}}-\frac{i M_{x}^{\prime}+j M_{y}^{\prime}}{T_{2}}
$$




\subsubsection{Continuous Wave EPR}

Continuous irradiation of a sample leads to a steady state condition for the net magnetization (in terms of magnetic field)

$$
\begin{aligned}
& M_{x}^{\prime}=\frac{\gamma_{e} B_{1} T_{2}^{2}\left(B_{o}-B\right) M}{1+T_{2}^{2}\left(B_{o}-B\right)^{2}+\gamma_{e}^{2} B_{1}^{2} T_{1} T_{2}} \\
& M_{y}^{\prime}=\frac{\gamma_{e} B_{1} T_{2} M}{1+T_{2}^{2}\left(B_{o}-B\right)^{2}+\gamma_{e}^{2} B_{1}^{2} T_{1} T_{2}} \\
& M_{z}^{\prime}=\frac{1+T_{2}^{2}\left(B_{o}-B\right)^{2}}{1+T_{2}^{2}\left(B_{o}-B\right)^{2}+\gamma_{e}^{2} B_{1}^{2} T_{1} T_{2}}
\end{aligned}
$$

In a CW-EPR experiment, the frequency is held constant and the field swept due to instrumentation constraints as it is easier and more stable to tune a resonator to the frequency band produced by the source and sweep the field. The signal is modulated at $100 \mathrm{kHz}$ to reduce background noise, and only the modulated part is detected by phase sensitive detection. Since the modulated signal is in the xy plane, magnetization in the zdirection is not detected. The absorption lineshape is Lorentzian, but what is recorded is the first derivative due to the phase sensitive detection. The Lorentzian lineshape is independent of the magnetic field at low power but is dependent on $T_{1}$ and $T_{2}$ relaxation, which will broaden the line. Because $\mathrm{T}_{1}$, the time necessary for the spin system to return to thermal equilibrium, is longer than $T_{2}$, the loss of phase coherence in the xy-plane, $T_{2}$ effects dominate for a protein in solution. Therefore, $\mathrm{T}_{2}$ determines the Lorentzian line width. Heisenberg spin exchange, which occurs when two spins are close enough for 
orbital overlap and, thus, an exchange in magnetization, and the dipolar interaction, both decrease $\mathrm{T}_{2}$ and lead to broadening.

\subsubsection{Power Saturation}

Relaxation effects determine the power absorbed by a sample

$$
P=\frac{B \gamma^{2} B_{1}^{2} M T_{2}}{1+T_{2}^{2} \gamma^{2}\left(B_{o}-B\right)^{2}+\gamma_{e}^{2} B_{1}^{2} T_{1} T_{2}}
$$

where $\gamma^{2} B_{1}^{2} T_{1} T_{2}$ is the saturation factor $P_{2}$. The peak-to-peak amplitude $(A)$ of the first derivative line increases linearly at low power by $\sqrt{P}$

$$
A=\frac{c \sqrt{P}}{\left(1+c^{2} P \gamma^{2} T_{1} T_{2}\right)^{\varepsilon}}
$$

where $c$ is a resonator constant and $\varepsilon$ is $\frac{3}{2}$ due to homogeneous broadening. Saturation occurs when there is deviation from the $\sqrt{P}$ dependence; power is absorbed by the sample more quickly than it can relax to equilibrium, which is a function of $T_{1}$ relaxation as $\frac{1}{T_{1}}$.

In the power saturation experiment, a parameter $P_{\frac{1}{2}}$ is measured, which is the power at which the peak-to-peak amplitude is half of the maximum in the absence of relaxation. $P_{\frac{1}{2}}$ is proportional to $\frac{1}{T_{1} T_{2}}$ and can estimate $T_{1}$ through paramagnetic relaxation enhancement. The addition of a second paramagnetic species decreases relaxation times through Heisenberg spin exchange. Since $T_{2}$ is faster than $T_{1}, T_{2}$ relaxation enhancement has a negligible effect, and $\mathrm{T}_{1}$ can be indirectly measured.

\subsubsection{Double electron-electron resonance}

Distances between two paramagnetic centers can be extracted through double electron-electron resonance (DEER), which is a two-frequency spin echo experiment. 
The sample is irradiated by short, intense pulses that are chosen by their tip angles; a $90^{\circ}$ $\frac{\pi}{2}$ pulse moves the net magnetization into the xy-plane for detection, and a $180^{\circ} \pi$ pulse inverts the net magnetization. DEER typically uses a 4-pulse sequence, although 5 and 7pulse sequences have recently been developed (Figure 3.1).

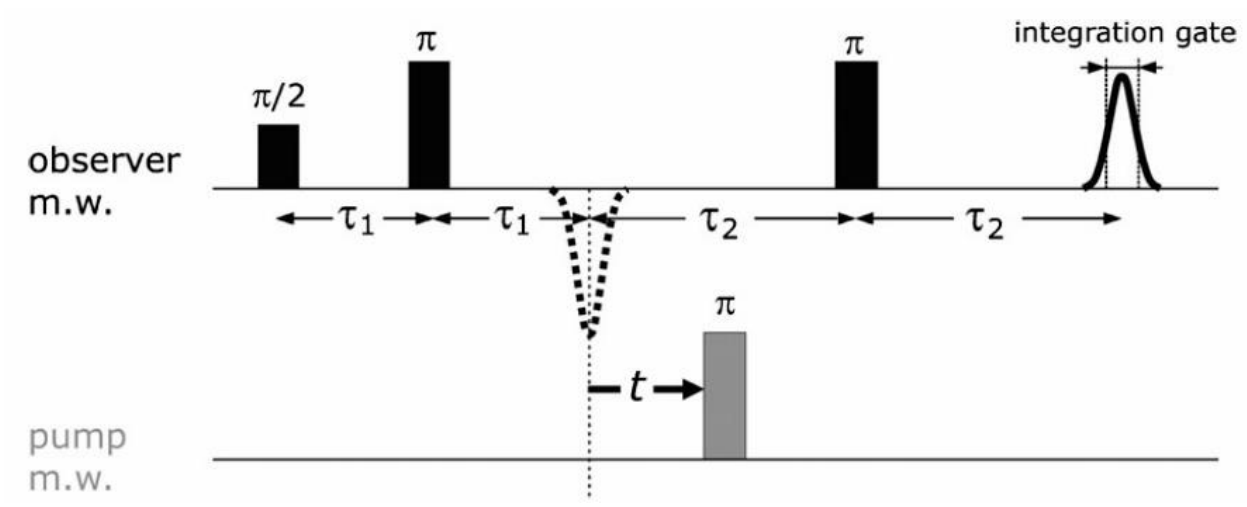

Figure 3.1. Pulse sequence for the 4-pulse DEER experiment. The $\pi / 2$ pulse tips the magnetization into the xy-plane. After a fixed delay $\tau_{1}$, a $\pi$ pulse reestablishes phase coherence, creating an undetected spin echo. The time of the pump pulse $t$ is varied, and after another observer $\pi$ pulse and a fixed delay $\tau_{2}$, the width of the echo at half height is recorded (Jeschke and Polyhach, 2006).

In the 4-pulse experiment, a $\frac{\pi}{2}$ pulse tips the net magnetization into the xy-plane and establishes phase coherence for the observer (A) spins. However, due to $\mathrm{T}_{2}$ relaxation, spins lose phase coherence after a time $\tau_{1}$, and the net magnetization in the xyplane decays to zero. The phase coherence can be reestablished with the application of a $\pi$ pulse, creating a spin echo, which is called a Hahn echo (Figure 3.2). A $\pi$ pump pulse inverts the pump (B) spins and, therefore, the dipolar coupling frequency, which creates a phase lag in the (A) spins. The phase lag is affected both by the static dipolar frequency contribution and the timing of the pump pulse, so the timing of the pump pulse is varied to extract the coupling from the timing. The resulting signal is the echo intensity modulation by the time-varied pump pulse and is proportional to the dipolar 
coupling.Long distances have low frequency oscillations due to the $\mathrm{r}^{-3}$ dependence, so the measurable distance range is approximately $2-8 \mathrm{~nm}$.

$$
\omega_{d d}=\frac{1}{r^{3}} \frac{\mu_{0} g_{1} g_{2} \beta_{e}^{2}}{4 \pi h}\left(1-3 \cos ^{2} \theta\right)
$$
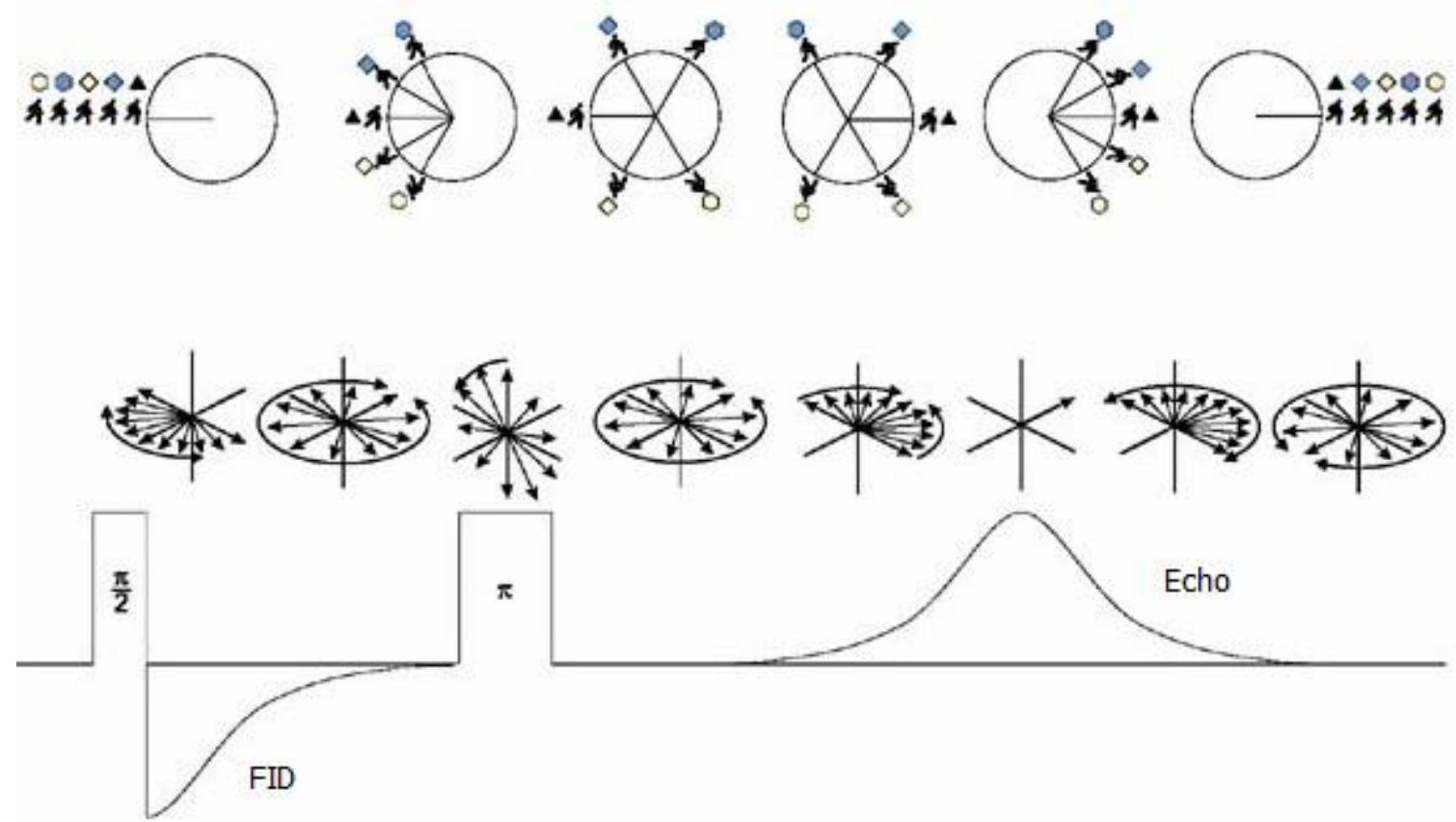

Figure 3.2. Loss and reestablishment of phase coherence. After application of the $\pi / 2$ pulse, spin packets (represented by the runners) interact with nearby spin packets and change frequency, losing coherence. Phase coherence is reestablished by the application of a $\pi$ pulse (Weber and Heiss, 2001).

The echo decay $\left(\mathrm{T}_{\mathrm{m}}\right)$ controls the length of signal acquisition and is more complex than $\mathrm{T}_{2}$ with additional contributions from spectral diffusion, spin diffusion, and instantaneous diffusion. Spectral diffusion is frequency fluctuations over time; for example, a spin at the observer frequency changes, and is no longer contributing to the observer signal. Instantaneous diffusion is the frequency shift of nearby spins due to inversion and occurs at high spin concentrations. Spin diffusion is the loss of net magnetization to other parts of the sample instead of the lattice. All of these shorten the 
$\mathrm{T}_{\mathrm{m}}$ and, therefore, the time the signal evolution can be recorded; the resulting spectrum of the signal evolution is called the time domain.

Time domain data contain both intramolecular $(F(t))$ and intermolecular $(B(t))$

dipolar interactions

$$
V(t)=F(t) B(t)
$$

and the intramolecular signal decays to $1-\Delta$ (modulation depth).

$$
V(t)=\{1-[1-\Delta]\} B(t)
$$

In order to generate a distance distribution between the two spins of interest, the intermolecular background interactions must be subtracted. The background is described by a simple exponential when the sample distribution is homogenous,

$$
B(t)=e^{-k t^{d / 3}}
$$

where $k$ is the spin density and $d$ is the background. As long as the intramolecular distance is $<4 \mathrm{~nm}$, the early part of the time domain will contain $D(t)$, and the later part of the time domain will contain $B(t)$. The background is difficult, if not impossible, to subtract completely at longer distances, because it contributes to the entire time domain (Jeschke, 2013). Background subtraction is also dependent on the dipolar evolution time. If the dipolar evolution time is sufficiently long,

$$
t_{\max } \geq \frac{8 \pi h r_{A B}^{3}}{g_{A} g_{B} \mu_{O} \mu_{B}^{2}}
$$

and background subtraction is over the $2^{\text {nd }}$ half of the time domain, a good fit can be obtained (Jeschke and Polyhatch, 2007). 
Fitting time domain data is an ill-posed mathematical problem. Several fitting algorithms have been developed to constrain physically irrelevant solutions. The most widely used is the Tikhonov regularization, which stabilizes the solution by calculating regularization parameters $(\alpha)$ using the L-curve criterion. The L-curve is a plot of log $\eta(\alpha)$ (smoothness of the distribution),

$$
\eta(\alpha)=\left|\frac{d^{2}}{d r^{2}} P(r)\right|_{\alpha}^{2}
$$

where $\mathrm{P}(r)$ is the distance distribution, vs. $\log \rho(\alpha)$ (mean square deviation)

$$
\rho(\alpha)=|S(t)-D(t)|_{\alpha}^{2}
$$

where $S(t)$ is the simulated distribution. The plot is L-shaped for data with low signal to noise and distributions with narrow peaks, and the best fit is the value that balances resolution and smoothness, which is at the corner of the curve. Larger $\alpha$ values to the right of the corner oversmooth the distribution, leading to excessive broadening. Smaller values above the corner undersmooth the distribution and create artefacts. Noisy data or broad distributions can distort the curve, so $\alpha$ should be chosen based on the correct smoothness of the narrowest peak.

\subsection{EPR Applications and Interpretation}

\subsubsection{Site Directed Spin Labeling}

Most proteins do not have the paramagnetic center necessary to perform EPR experiments. Site directed spin labeling (SDSL) allows a spin label, typically 1-oxy2,2,5,5-tetramethylpyrroline-3-methylmethanethiosulfonate (MTSL), to be covalently attached at the site of interest (Berliner et al., 1982). The native residue is mutated to a cysteine, and the spin label is attached via a disulfide bond, creating a new side chain 
designated R1 (Figure 3.3). There is not free rotation around all five bonds in the modified side chain (Figure 3.3). Simulations and X-ray crystallography have shown that rotation is limited by back bonding between the first sulphur (typically referred to as $\mathbf{S}_{\delta}$ ) and $\mathrm{C}_{\alpha}$, restricting rotational motion to the $\chi_{4}$ and $\chi_{5}$ bonds in models that represent spin label motion on $\alpha$-helical sites, such as the Wobble model (Columbus et al., 2001).

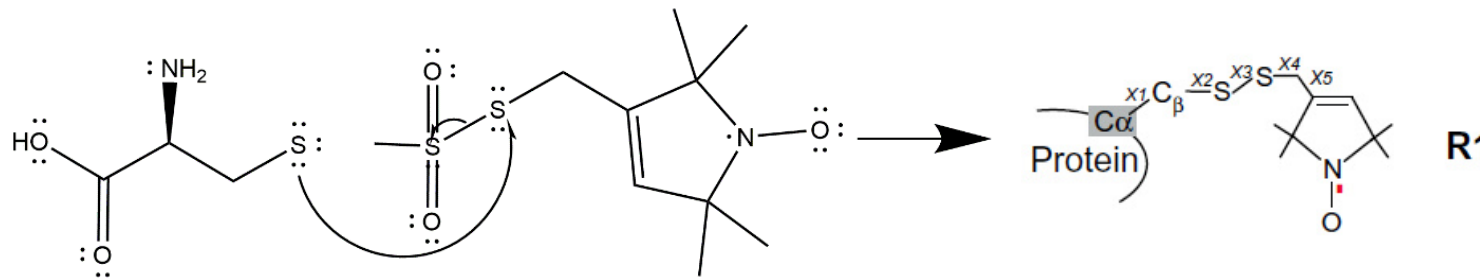

Figure 3.3. The site directed spin labeling reaction with MTSL. A disulfide bond is formed between MTSL and the deprotonated cysteine side chain, creating the modified side chain, $\mathrm{R} 1$. The reaction was drawn in ChemDraw, and the product adapted from Altenbach et al., 2015.

MTSL is stable, selective, and sensitive. The spin label does not react with other side chains, and attachment of the spin label has negligible effects on protein folding and function. The sensitivity of EPR experiments is approximately 1000x greater than NMR due to the much larger magnetic moment of electrons and higher experimental frequencies (Mchaourab et al., 1996; Altenbach et al., 1990). Continuous wave (CW) and pulse EPR can provide information on structure, dynamics, and topology in native and native-like environments (Figure 3.4).

\subsubsection{CW-EPR}

CW spectra provide information on secondary and tertiary structure, protein backbone dynamics, and the local environment of the spin label, which can report on conformational exchange events. These have been extensively described for the model system T4 lysozyme (Mchaourab et al., 1996; Guo et al., 2000; Columbus et al., 2001) and, to a lesser extent, $\beta$-barrel proteins (Freed et al., 2011). Most CW experiments are at 


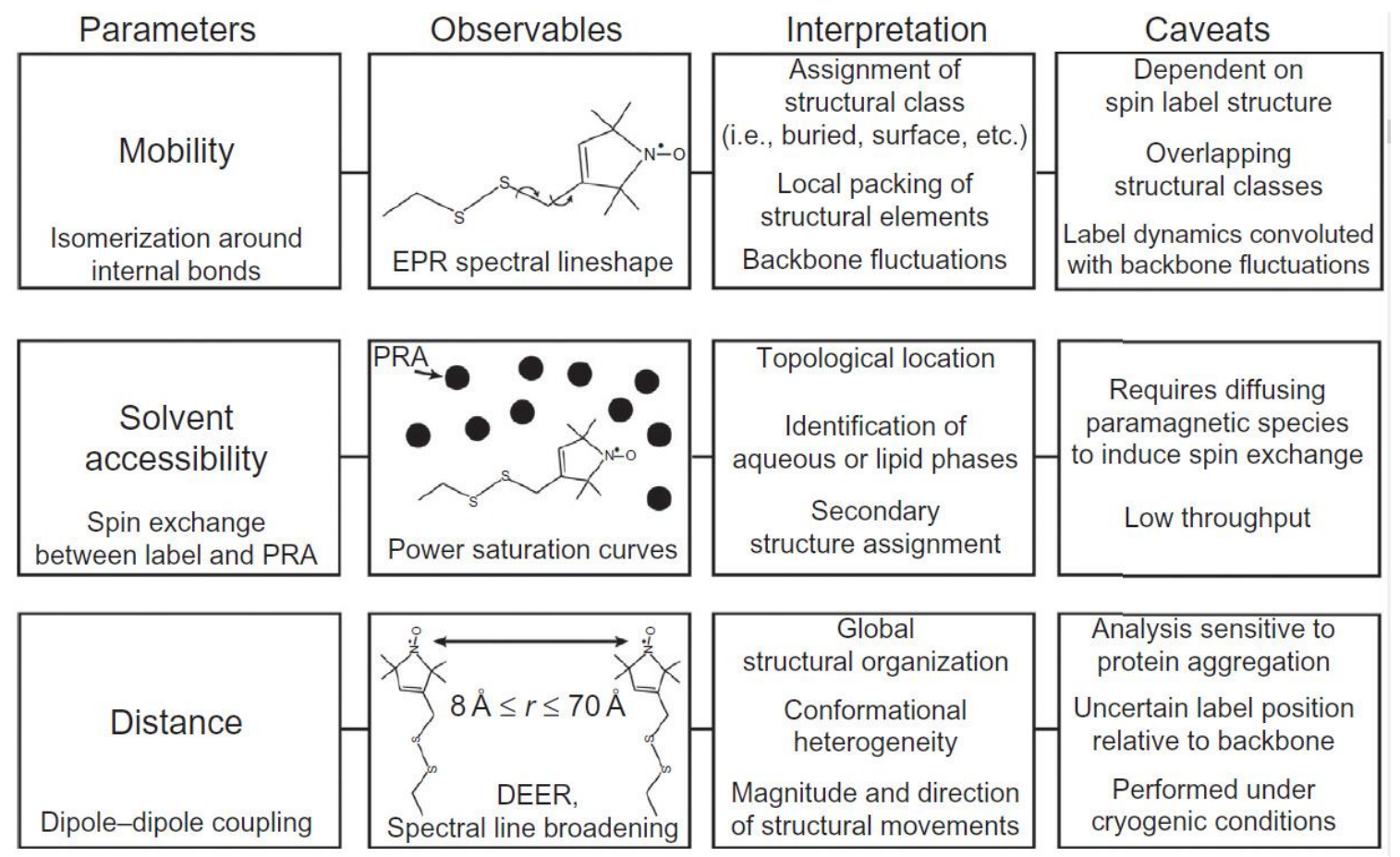

Figure 3.4. A summary of the most common EPR experiments. The use of CW lineshapes, power saturation, and DEER are outlined (Claxton et al.; 2015)

X-band $(9-10 \mathrm{GHz})$; at these frequencies, the spectra report on the rotational motion of the spin label in the pico to nanosecond timescale (fast: $<2 \mathrm{~ns}$, intermediate: $2-30 \mathrm{~ns}$, or slow: >30 ns) (Van et al., 1974; Kivelson, 1960). Quantitative information on nitroxide motional modes can be extracted through simulations. While simulations are useful for describing the motion of R1 and determining whether multiple motional modes to describe a spectrum, secondary structure can often be assigned from simple spectral features. Due to the magnitude of backbone fluctuations and the interaction of the spin label with nearby side chains, there are characteristic lineshapes for R1 based on secondary structure (Figure 3.5) (Lietzow et al., 2004; Mchaoraub et al., 1996). Sites with less ordered secondary structure will have larger backbone fluctuations than sites with more ordered secondary structure. Furthermore, the inverse central line width $\left(\Delta H^{-1}\right)$ provides a measure of mobility at noninteracting (loop or helix surface) sites, and the 
correlation of $\Delta H^{-1}$ with the inverse second moment $\left.\left(<H^{2}\right\rangle^{-1}\right)$ provides structural information for not only those sites but tertiary contact and buried sites (Mchaoraub et al., 1996). Motionally restricted sites can also have resolved hyperfine extrema that allow overall splitting to be used for analysis. Most of the work has been done on solvent exposed sites. (Figure 3.6). However, R1 motion has been characterized to a lesser extent in hydrophobic environments (Kroncke et al., 2010). Spectra of membrane exposed sites are typically complex, multicomponent spectra with broader $\Delta H_{0}^{-1}$ as compared to solvent exposed sites (Figure 3.7).

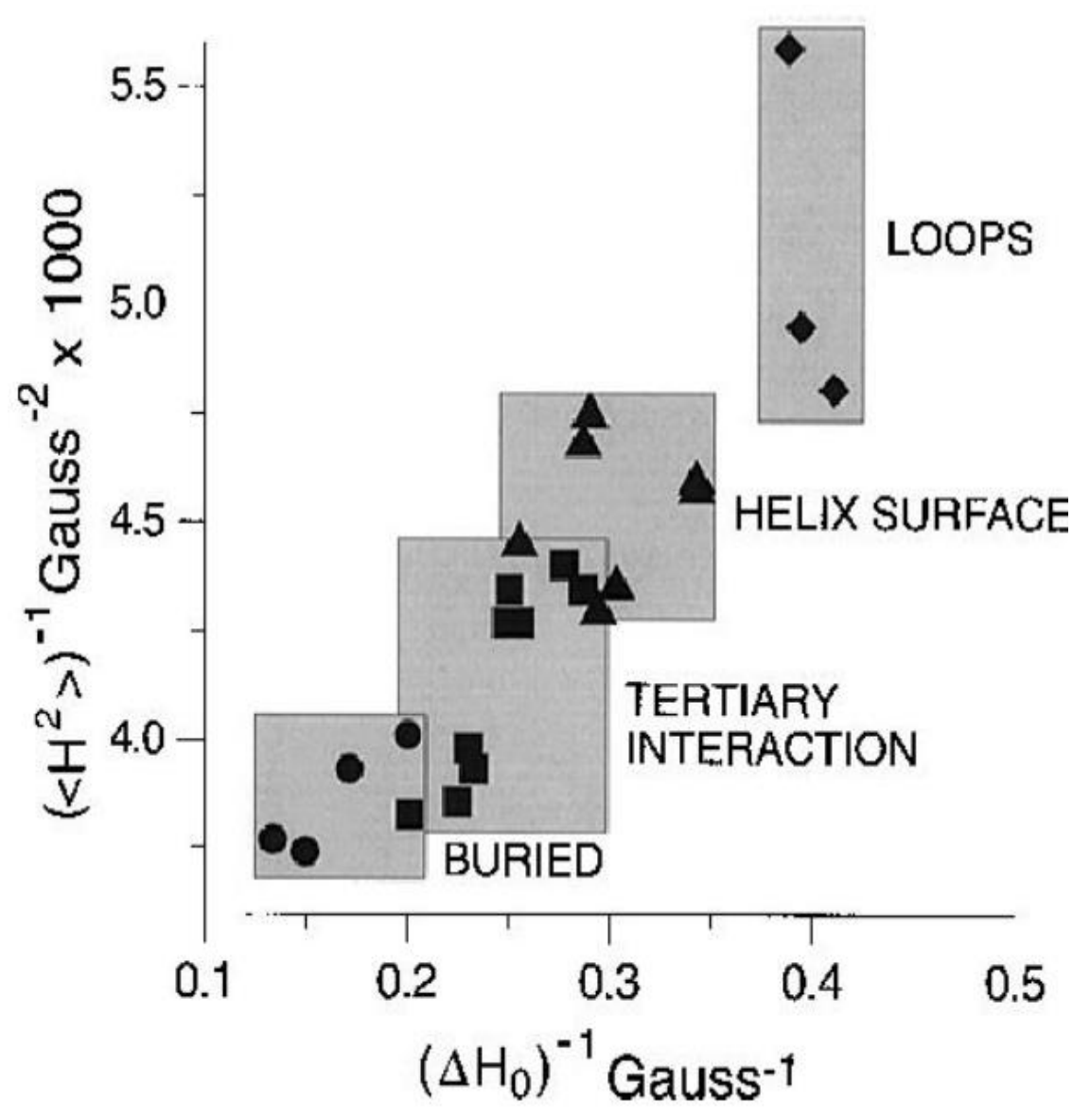

Figure 3.5. Secondary structure as determined by the second moment and inverse central line width (Mchaoraub et al., 

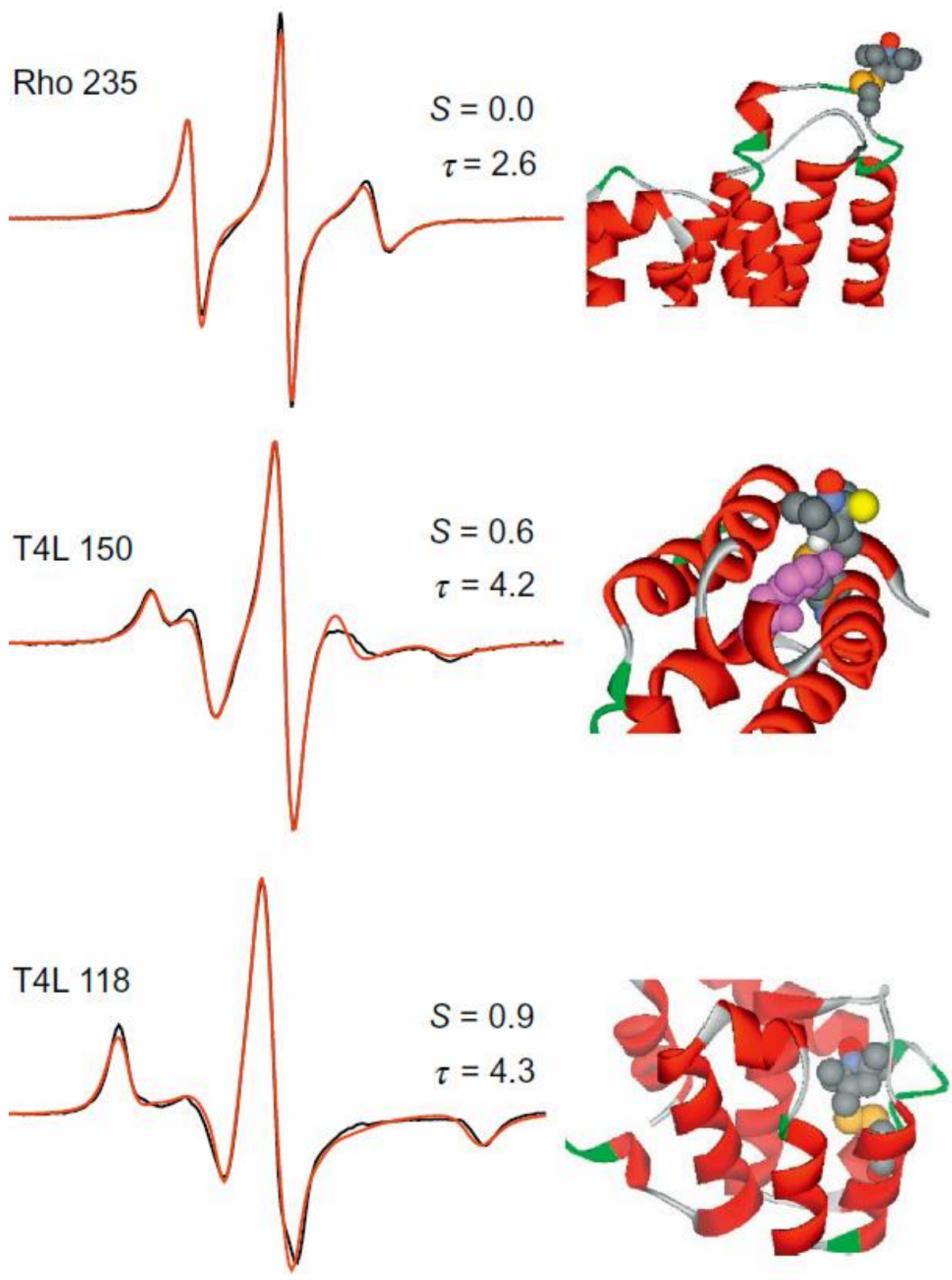

Figure 3.6. Characteristic lineshapes for loop, solvent exposed alpha helical, and buried sites. The R1 side chain at these sites typically undergoes simple anisotropic motion that is restrained by ordering (adapted from Altenbach et al., 2015). 


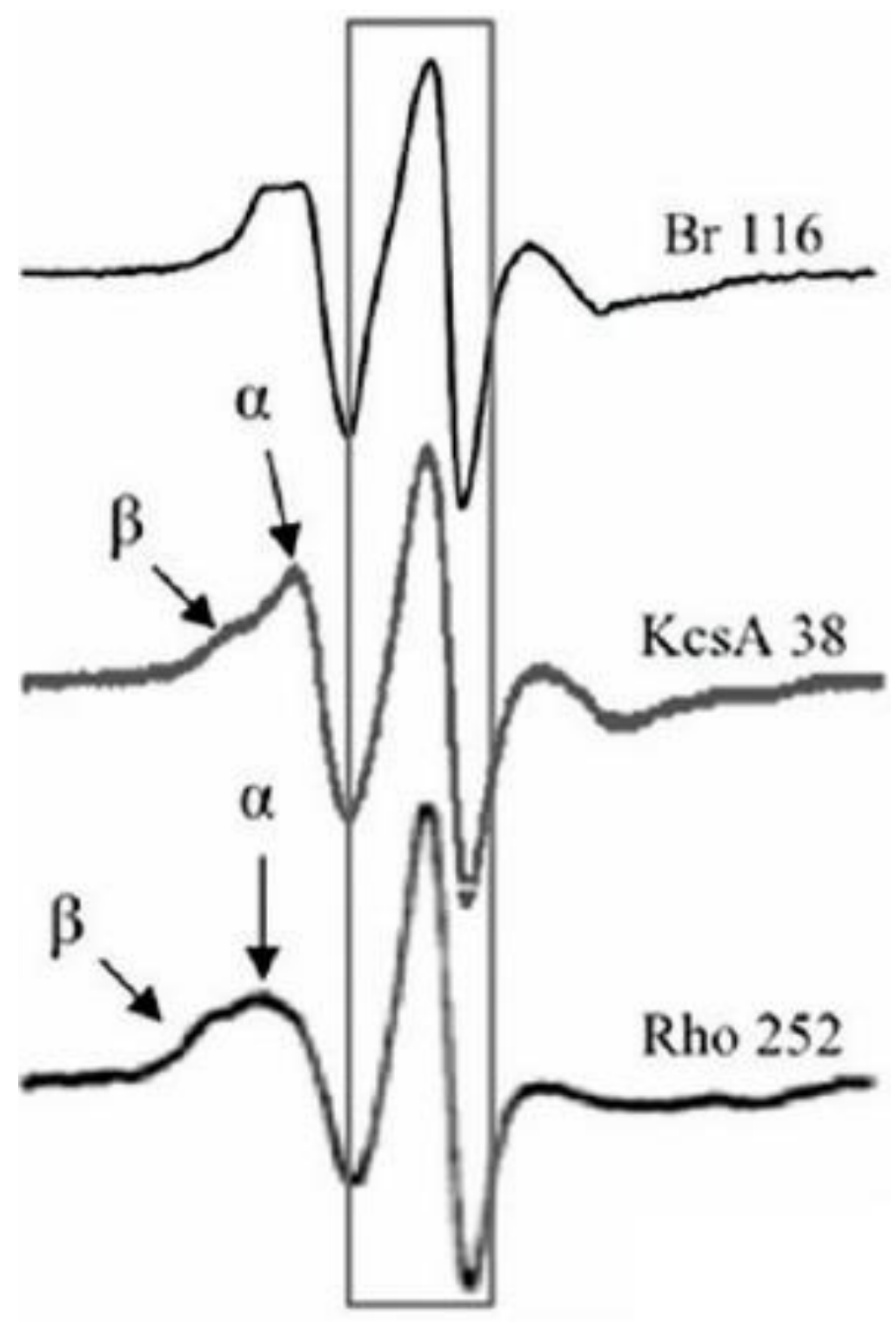

Figure 3.7. Alpha helical CW lineshapes in a hydrophobic environment (adapted from Kroncke et al., 2010). 
Multicomponent spectra arise when different environments are sampled, either due to rotameric states of the label or conformational fluctuations of the protein (Figure 3.8) (Guo et al., 2008; Bridges et al., 2010). The addition of protective osmolytes such as PEG or sucrose has been shown to modulate conformational populations but not rotameric states (Lopez et al., 2009) and thus can be used to distinguish the source of components. Because the spin label is small, changes in the solvent accessible surface area are negligible among the rotameric states, so the spin label itself is not sensitive to osmolytes. Simulations can be used to estimate the relative conformational populations during a sucrose titration, allowing the calculation of $\Delta \Delta \mathrm{G}$ between the states (Freed et al., 2010; Fanucci et al., 2003; Cafiso, 2014).
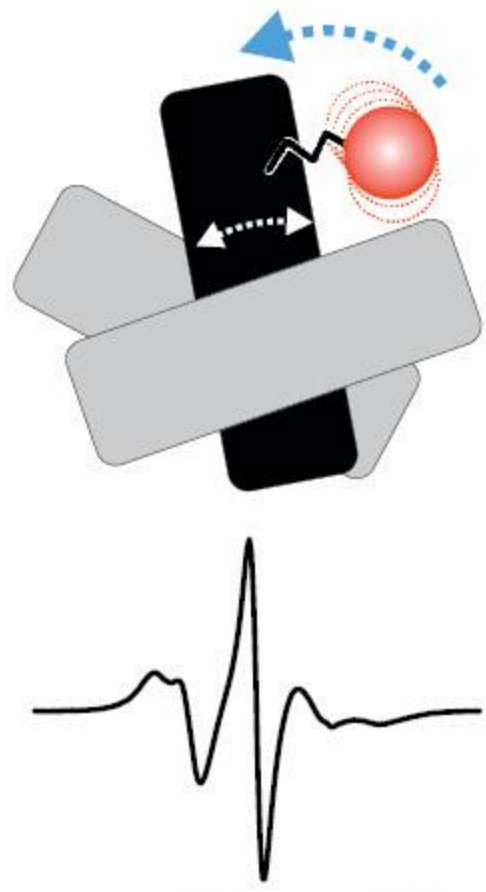

Weakly ordered
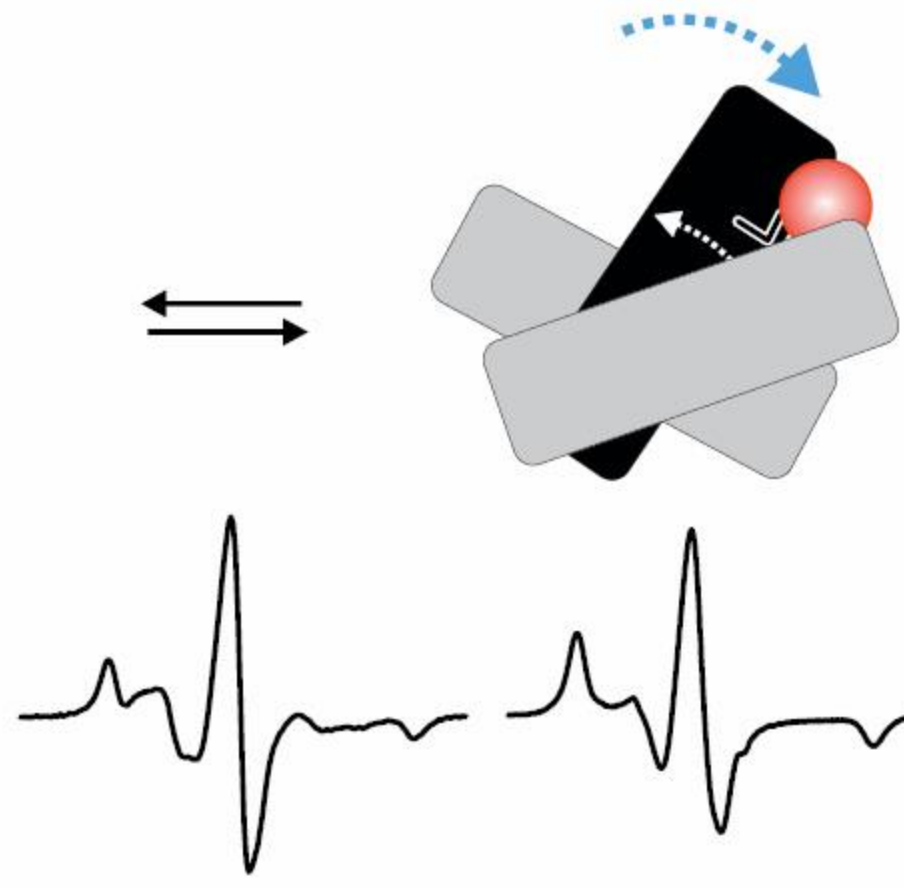

Two-component

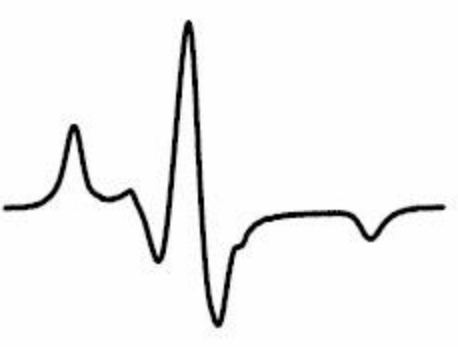

Immobilized

Figure 3.8. A two-component CW-EPR spectrum from conformational exchange. The spectrum is the sum of the components, weighted by conformational populations (Altenbach et al., 2015). 


\subsubsection{Power saturation}

Power saturation measures solvent accessibility by comparing the spin label's relaxation rates in the presence and absence of paramagnetic relaxation enhancers (PRA). The typical experiment uses $\mathrm{O}_{2}$ as the membrane soluble reagent and nickel (II) EDDA (NiEDDA) as the aqueous reagent. There is a concentration gradient of both of these species due to the increasing dielectric from the middle of the membrane to the headgroups (Figure 3.9). The collision frequency determines the change in relaxation rate and, therefore, the change in the amplitude of the central line in a CW spectrum. The central line of CW spectra is recorded at increasing powers, the amplitude of the line is plotted against the square root of the power, $\sqrt{P}$, and the curve is fit to determine the $P_{1 / 2}$. Nitrogen is used to purge oxygen from the sample so that the saturation behavior in the absence of other paramagnetic reagents may be recorded, and this is subtracted to calculate the accessibility parameters (П) for $\mathrm{O}_{2}$ and NiEDDA.

$$
\Pi=P_{1 / 2 P R A}-P_{1 / 2 N_{2}}
$$

The accessibility parameters are used to calculate the depth parameter $(\Phi)$

$$
\Phi=\ln \left(\Pi_{O_{2}} / \Pi_{N i E D D A}\right)
$$

which is positive for membrane sites and negative for aqueous sites. The depth parameter is related to the distance of the spin label to the phosphate in a lipid headgroup and it can be described by a hyperbolic tangent function that takes into account the behavior at the aqueous/membrane interface. Power saturation has been used to investigate SNARE membrane docking, membrane interfaces, and compare protein topology to X-ray crystal structures (Frazier et al., 2002; Sahu et al., 2015; Steed et al., 2013). 


\section{NiEDDAA}

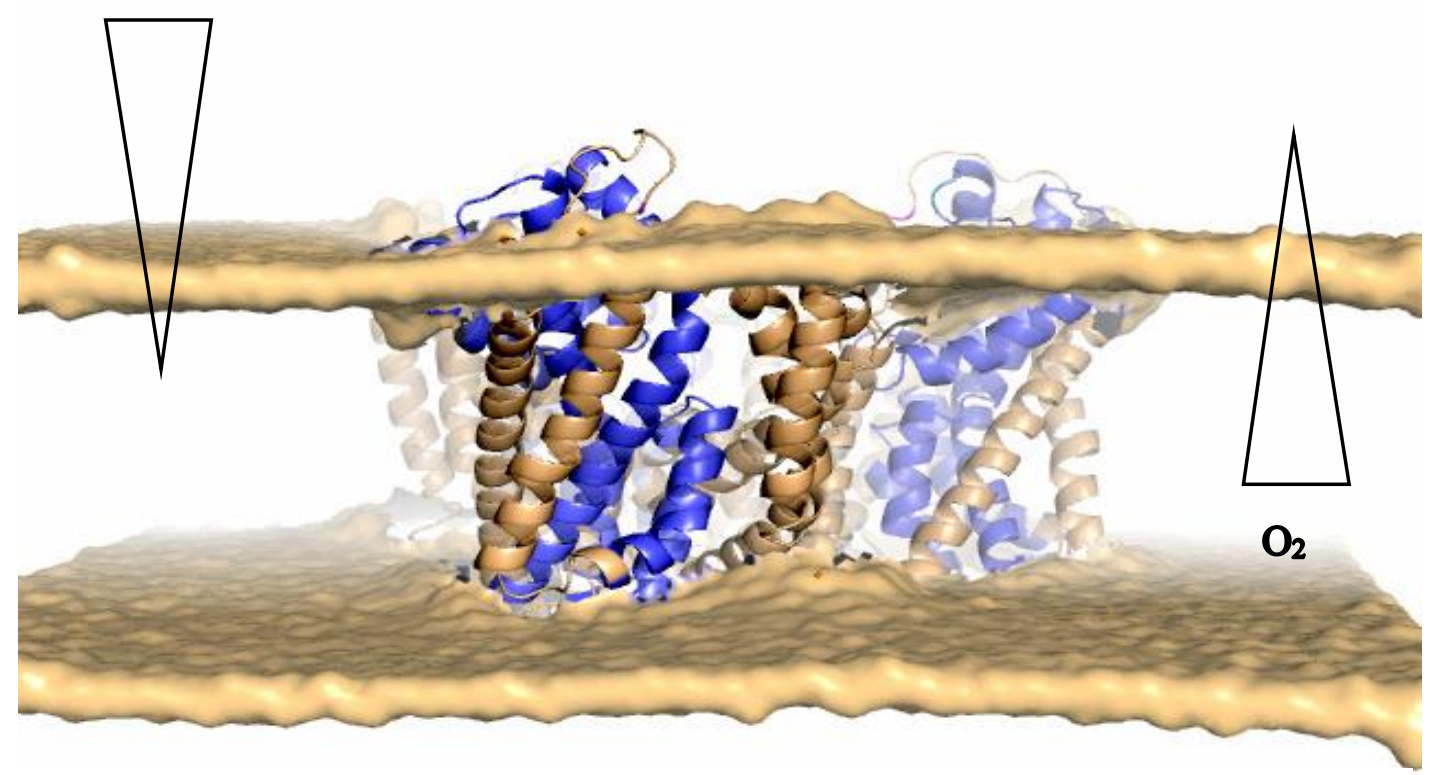

Figure 3.9. Power saturation schematic. $\mathrm{O}_{2}$ and NiEDDA gradients are shown on a model of $\mathrm{Glt}_{\mathrm{ph}}$ in a membrane (Adapted from MemProtMD).

\subsubsection{DEER}

DEER provides information that may complement X-ray crystallography and NMR. Large proteins can be studied in native-like environments, such as the lipid bilayer, and information can be gathered on very flexible or disordered regions. Distance distributions give information about protein conformational states, and ensembles of distributions can provide tertiary structure information. Comparisons to crystal structures can be made by simulating distance distributions using the program Mutiscale Modeling of Macromolecular systems (MMM), which employs a rotamer library to see if the static structure is sampled in native-like environments (Jeschke, 2009).

The raw DEER signal contains both the intramolecular dipolar interaction of interest and the background dipolar interactions that must be subtracted (Figure 3.10). 

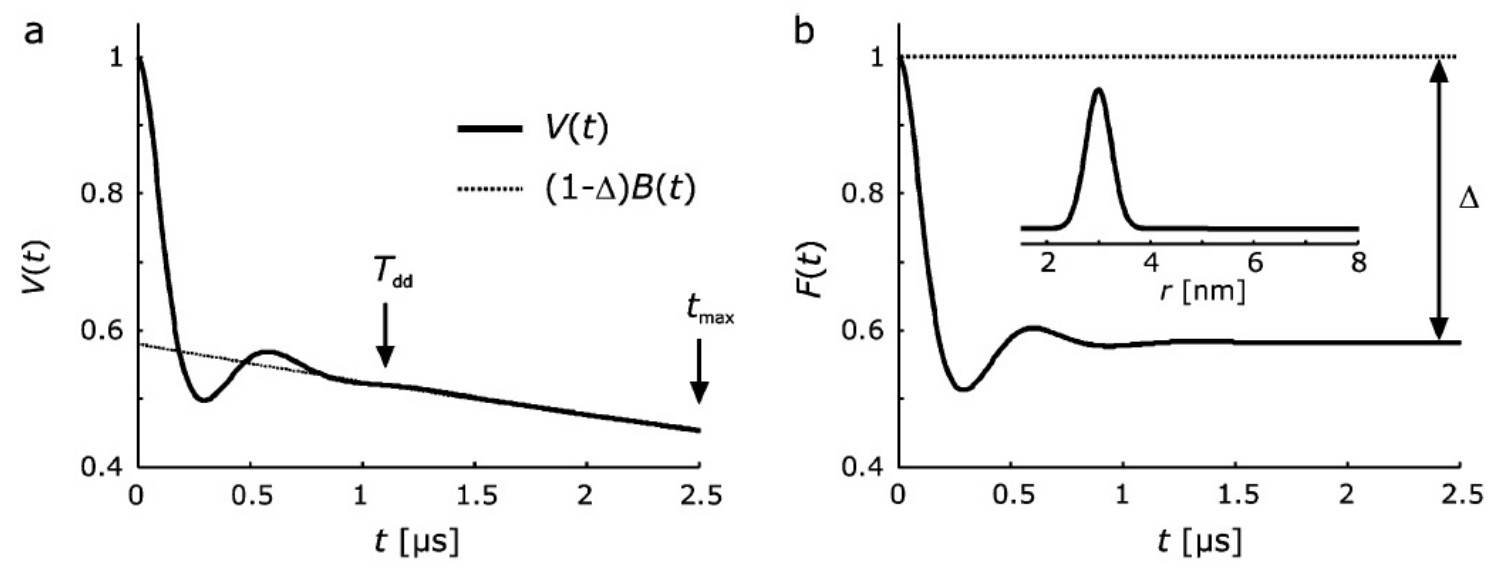

Figure 3.10. Subtraction of the background from the DEER dipolar evolution and the resulting form factor. a) The background $(1-\Delta) \mathrm{B}(t)$ is subtracted from the signal, $\mathrm{V}(t)$ to produce the form factor. $\mathrm{b})$ The form factor with the modulation depth $(\Delta)$ and resulting distance distribution (Jeschke and Polyhatch, 2007).

Distance distributions are calculated from the resulting form factor. Since fitting time domain data is an ill-posed problem, the Tikhonov regularization and the L-curve criterion constrain the solutions, and the best fit is determined by choosing the appropriate regularization parameter, $\alpha$ (Figure 3.11) (Jeschke and Polyhatch, 2007). After choosing the optimal $\alpha$, a validation procedure determines changes in the distribution at different background subtractions, dimensionality, and noise levels within user specified error bounds to determine the goodness of fit. The certainty of the average distances and width of the distribution is determined by the dipolar evolution time (Table 3.1). 


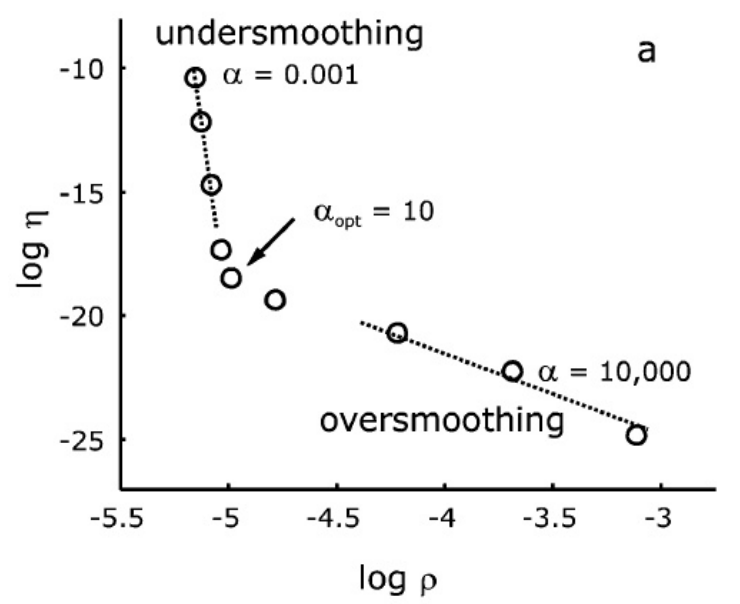

b
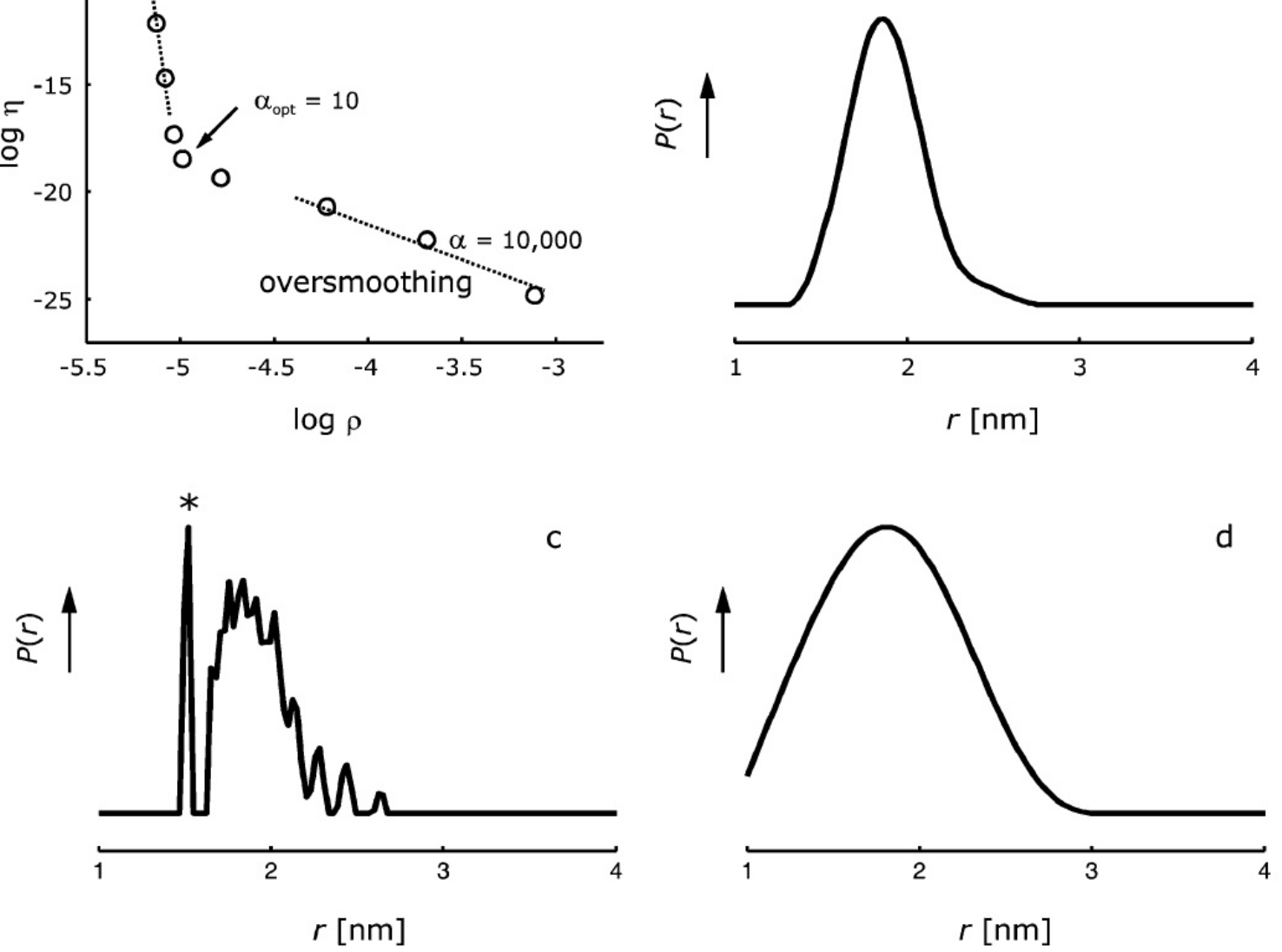

Figure 3.11. The effect of the regularization parameter on $\mathbf{P}(\mathbf{r})$. a) The L-curve generated through the Tikhonov regularization. b) Distance distribution generated through the optimal $\alpha$ shown in $a$. c) An $\alpha$ that is too small generates an undersmoothed distance distribution and artefacts. d) An $\alpha$ that is too large generates an oversmoothed distribution that is too broad (Jeschke and Polyhatch, 2007). 
Table 3.1. Resolution of DEER distributions at different dipolar evolution times (d2).

\begin{tabular}{|c|c|c|c|}
\hline $\mathbf{r}_{\mathrm{AB}}(\mathbf{n m})$ & $\begin{array}{c}\mathbf{d 2}(\boldsymbol{\mu} \mathbf{s})-\mathbf{0 . 1} \mathbf{~ n m} \\
\text { resolution }\end{array}$ & $\begin{array}{c}\mathbf{d 2}(\boldsymbol{\mu s})-\mathbf{0 . 2} \mathbf{~ n m} \\
\text { resolution }\end{array}$ & $\begin{array}{c}\mathbf{d 2}(\boldsymbol{\mu} \mathbf{s})-\mathbf{0 . 5} \mathbf{~ n m} \\
\text { resolution }\end{array}$ \\
\hline 2.0 & 1.0 & 0.5 & 0.2 \\
\hline 2.5 & 2.5 & 1.3 & 0.5 \\
\hline 3.0 & 5.2 & 2.6 & 1.0 \\
\hline 3.5 & 9.6 & 4.8 & 3.2 \\
\hline 4.0 & 16.4 & 8.2 & 5.2 \\
\hline 4.5 & 26.3 & 13.7 & 8.0 \\
\hline 5.0 & 40 & 20 & \\
\hline
\end{tabular}


Chapter 4

Results 


\subsection{Introduction}

Previous work has shown that Glt $\mathrm{ph}_{\mathrm{p}}$ exists simultaneously in both inward and outward facing conformations (Georgieva et al., 2013; Hanelt et al., 2013; Akyuz et al., 2015). However, it is not clear whether these states are in equilibrium. Furthermore, it is not clear whether the DEER distance distributions observed can be explained based upon the presence of label rotamers or whether additional structural heterogeneity exists within inward and outward facing conformations. Measurements made using different techniques at different sites have yielded contradictory results, and the source of these differences has not been resolved. To address these issues, we examined the major conformational states in Glt $\mathrm{ph}_{\text {by }}$ studying these states using a reconstituted system in which Glt $\mathrm{ph}_{\mathrm{p}}$ was spin-labeled at a single site. Because Glt $\mathrm{ph}_{\mathrm{p}}$ is a trimer, DEER experiments on this single-labeled protein yielded an intermonomer distance that reflected the major conformational states of the protein. One complicating factor in these measurements is that the transport domain of each monomer can act independently. A mixed asymmetric trimer in which inward and outward facing conformations are both populated has been crystallized (Verdon and Boudker, 2012). For many of these measurements, sites were chosen where the label was expected to have aqueous exposure and not be in tertiary contact, such as T375 and Q121, thereby minimizing the likelihood of perturbation by the label and making interpretation of the EPR spectra and prediction of the label rotamers more straightforward. 


\subsection{The inward facing conformation is stabilized by substrate in lipid bilayers}

Conformational exchange events can be captured using both DEER and CW-EPR. Multiple peaks in a distance distribution show resolved conformations, and peaks broader than 3-5 A can signify unresolved populations. Furthermore, the average distances can be compared to crystal structures to determine if the crystal structure is representative of the global structure in a biologically relevant environment.

Crystal structures have shown that the trimerization domain of $\mathrm{Glt}_{\mathrm{ph}}$ undergoes large scale conformational changes during the transport cycle. The transport domain moves approximately $18 \AA$ through the membrane to expose the substrate binding site to the intracellular side for substrate release. This leads to distance differences between sites in the transport domain during the transport cycle, and these differences can be measured using DEER. Conformation exchange also changes the local environment of the site, which can be measured using CW-EPR. However, sites in the trimerization domain are not expected to undergo conformational exchange during transport and are good control sites for these measurements. Since $\mathrm{Glt}_{\mathrm{ph}}$ is a homotrimer, one mutation provides three sites for spin labeling, allowing DEER measurements. Three-spin short distance artifacts have been previously shown to not affect the distance distributions (Hanelt et al., 2013).

V176 is on TM 5 in the trimerization domain and is, therefore, a good control site for DEER measurements. The mean distance and the width of the distribution did not change with either substrate or inhibitor addition, and there was not sucrose modulation. Therefore, this site was not undergoing conformational exchange or changes in solvent exposed surface area during the transport cycle, which was expected based on both the crystal structures and simulations using rotamer library analysis in the program 
Multiscale Modeling of Macromolecules, or MMM (Figure 4.1) (Polyhatch et al., 2011).
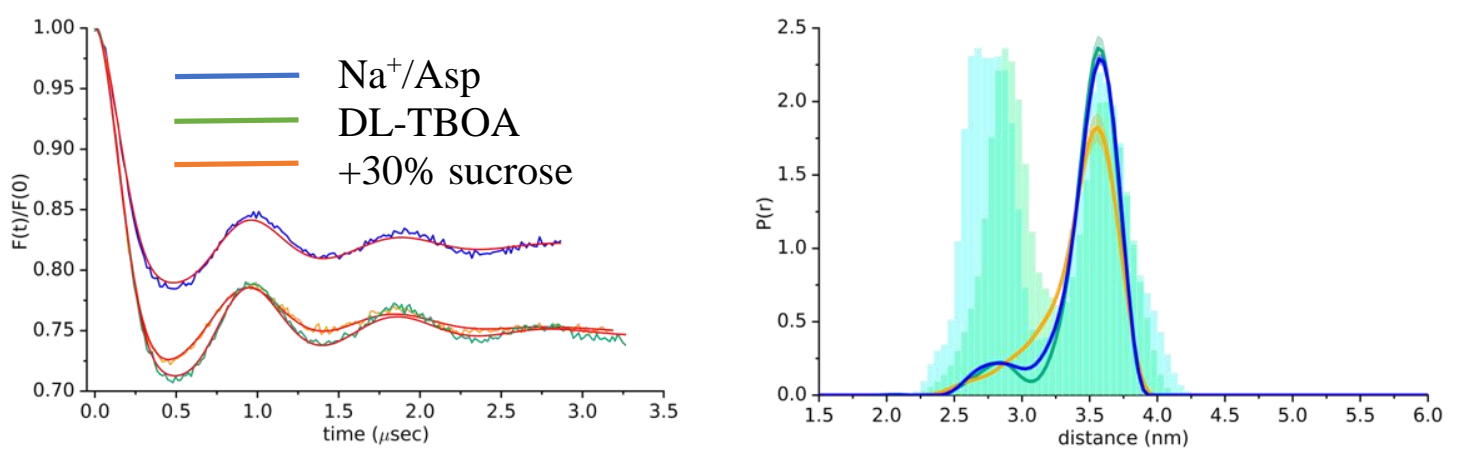

Figure 4.1. DEER distance distributions for V176R1 in the trimerization domain. Distributions were obtained by Tikhonov regularization, and substrate states are detailed by the legend. The site is well behaved and well defined with a peak width of $3 \AA$ and agrees well with the simulated distance distributions of the outward (light blue) and inward (light green) facing conformations

The average distance of $35 \AA$ agreed with well with the crystal structure, and the peak width at half height was $3 \AA$, which is typical for a defined distance at a well-behaved site (Table 4.1). However, the MMM distribution shows two peaks of equal intensity. The peak at $27 \AA$ was due to spin label rotamers that were not part of the experimental distance distribution. The $\mathrm{CW}$ spectra also supported a well-defined site not undergoing conformational exchange because the lineshape did not change when substrate or inhibitor were added (Figure 4.2).

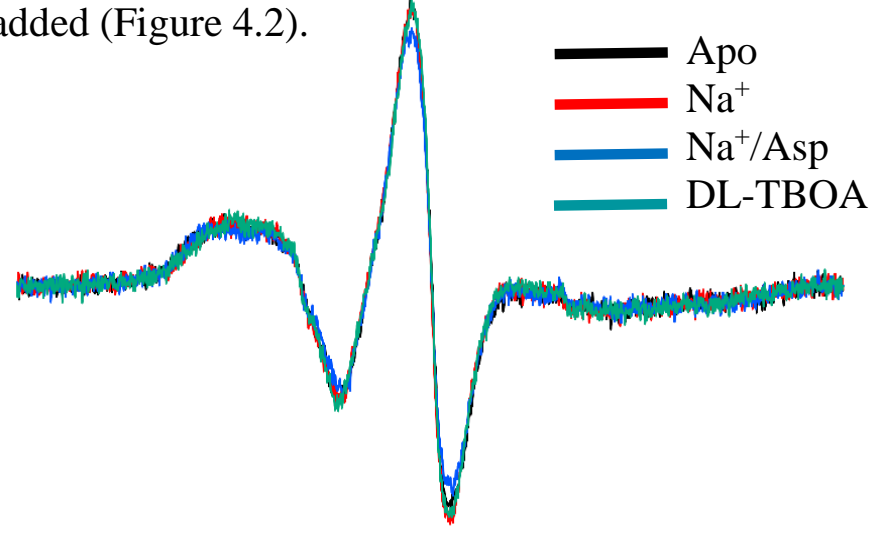

Figure 4.2. CW-EPR spectra at V176R1. The lineshape did not change from substrate free to substrate or inhibitor bound protein, which was expected for a site in the trimerization domain. 
The crystal structures show distance differences between the outward and inward facing conformations at T375R1, which is on a small loop segment between TM 8 and HP2 (Figure 4.3). These distances were optimal for DEER measurements since the differences were large and in the effective range of 20-60 A.
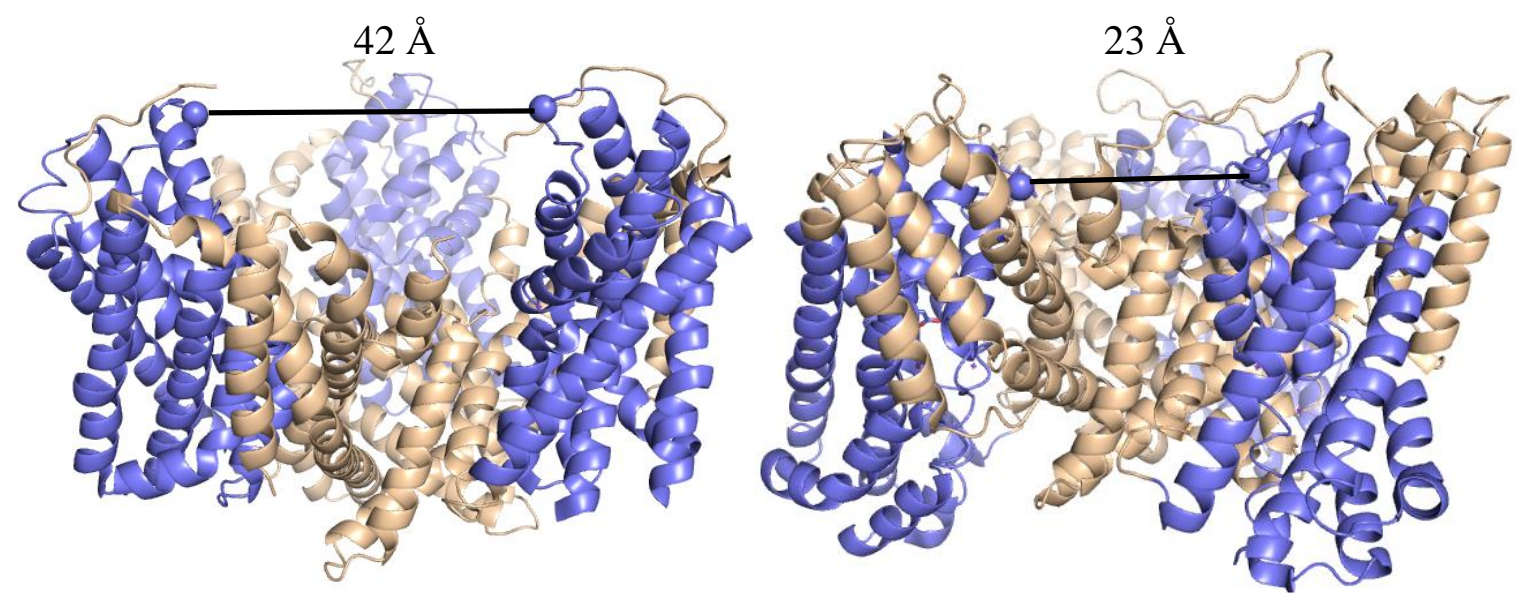

Figure 4.3. $\mathrm{C}_{\alpha}-\mathrm{C}_{\alpha}$ distances for $\mathrm{T} 375$ in the outward (left) and inward (right) facing conformations. The trimerization domain is tan. The transport domain is blue, and T375 is shown as a sphere.

The distance distributions in the substrate free and $\mathrm{Na}^{+}$bound states were broad and characterized by multiple peaks, indicating conformational heterogeneity (Figure 4.4 a,b). The peaks for the substrate free and $\mathrm{Na}^{+}$bound states at $26 \AA$ had a width of approximately $10 \AA$, and the peaks around $44 \AA$ had widths greater than $5 \AA$ (Table 4.1 ), which are significantly larger than at the well-behaved control site V176R1. $\mathrm{Na}^{+} / \mathrm{Asp}$ shifted the distribution to the inward facing conformation, and DL-TBOA to the outward facing conformation, as shown by rotamer library analysis of the respective crystal structures (Figure $4.4 \mathrm{c}, \mathrm{d}$ ). This contradicted single molecule FRET results that show stabilization of the outward facing conformation in the presence of $\mathrm{Na}^{+} / \mathrm{Asp}$ at this site (Akyuz et al., 2013; Akyuz et al., 2015). $\mathrm{Na}^{+} /$Asp also narrowed the inward facing peak to $6 \AA$, suggesting reduced conformational heterogeneity when compared to the substrate 
free and $\mathrm{Na}^{+}$bound states (Figure 4.4, Table 4.1). This was in contrast to the broad distance distribution for inhibitor bound protein, which suggested that there was more conformational heterogeneity in the outward facing conformation than the inward facing conformation (Figure $4.4 \mathrm{~d}$ ).

Table 4.1. Mean distances and peak widths for DEER distance distributions at V176R1, T375R1, and T375R1p. The distances and peak widths were determined using Long Distances.

Peak 1 Peak $1(\AA) \quad$ Width $(\AA) \quad \operatorname{Peak} 2(\AA) \quad$ Width $(\AA)$

\begin{tabular}{|c|c|c|c|c|c|}
\hline \multirow{2}{*}{\multicolumn{6}{|c|}{ V176R1 }} \\
\hline & & & & & \\
\hline \multirow{8}{*}{$T 375 R 1$} & $\mathrm{Na}^{+} / \mathrm{Asp}$ & 35 & 3 & - & - \\
\hline & $+30 \%$ sucrose & 35 & 4 & - & - \\
\hline & DL-TBOA & 36 & 3 & - & - \\
\hline & & & & & \\
\hline & Apo & 26 & 10 & 41 & 8 \\
\hline & $+30 \%$ sucrose & 27 & 11 & 42 & 7 \\
\hline & $\mathrm{Na}^{+}$ & 26 & 11 & 44 & 6 \\
\hline & $+30 \%$ sucrose & 27 & 9 & 44 & 5 \\
\hline \multirow{13}{*}{ T375R1p } & $\mathrm{Na}^{+} / \mathrm{Asp}$ & 22 & 6 & 36 & 5 \\
\hline & $+30 \%$ sucrose & 22 & 6 & 34 & 9 \\
\hline & DL-TBOA & - & - & 39 & 9 \\
\hline & $+30 \%$ sucrose & - & - & 38 & 14 \\
\hline & & & & & \\
\hline & Apo & 20 & 7 & 32 & 9 \\
\hline & $+30 \%$ sucrose & 21 & 6 & 31 & 9 \\
\hline & $\mathrm{Na}^{+}$ & 20 & 7 & 32 & 7 \\
\hline & $+30 \%$ sucrose & 21 & 7 & 34 & 6 \\
\hline & $\mathrm{Na}^{+} / \mathrm{Asp}$ & 21 & 8 & - & - \\
\hline & $+30 \%$ sucrose & 21 & 10 & - & - \\
\hline & DL-TBOA & - & - & 40 & 15 \\
\hline & $+30 \%$ sucrose & - & - & 42 & 13 \\
\hline
\end{tabular}

The broad distance distributions seen by DEER could be due to conformational exchange or spin label rotamers. The addition of protective osmolytes can determine which is contributing to the distribution and provide information on conformational exchange among states in equilibrium. Sucrose modulation of conformational exchange at T375R1 was substrate dependent. The distribution was shifted toward the inward facing conformation when $\mathrm{Na}^{+}$was bound (Figure $4.4 \mathrm{~b}$ ). 

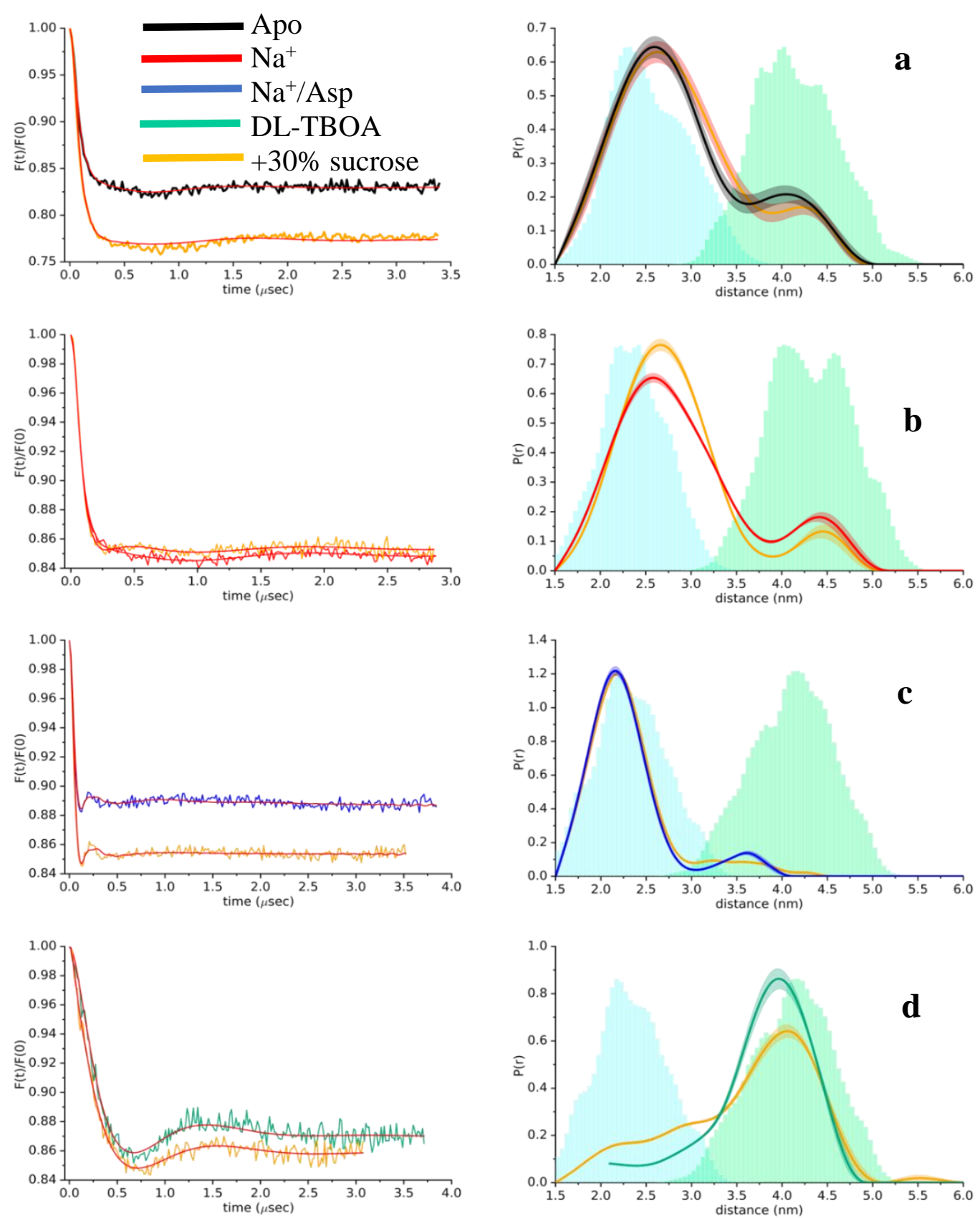

Figure 4.4. DEER distance distributions and rotamer library analysis for T375R1. The background subtracted time domain data are on the left, and the distance distributions are on the right. Distance distributions were obtained by Tikhonov regularization and compared to rotamer library analysis of the outward (green histogram) and inward (blue histogram) facing crystal structures. 
The small shift toward the inward facing conformation in the DL-TBOA bound state was determined to be an artifact based on peak suppression in DEER Analysis (Figure $4.4 \mathrm{~d}$ ) (Jeschke, 2013). The $\mathrm{Na}^{+} / \mathrm{Asp}$ and substrate free states were not modulated by sucrose (Figure $4.4 \mathrm{c}$ ). Since the $\mathrm{Na}^{+} / \mathrm{Asp}$ bound state was already in the inward facing conformation, this result was not surprising (Figure $4.4 \mathrm{c}$ ). However, distribution shifts were expected for the substrate free protein (Figure $4.4 \mathrm{a}$ ).

Conformational populations were calculated from the areas under each peak in the distributions with the assumption of two conformations. However, it was likely that the broad peak at $26 \AA$ in the substrate free and $\mathrm{Na}^{+}$bound states corresponded to more than one unresolved conformation since this peak was broader than the peak generated through rotamer library analysis. Under the assumption that a large part of the population at the shorter distance corresponds to the inward facing conformation, the addition of $\mathrm{Na}^{+}$ shifted the population away from the inward facing conformation. The substrate free protein had $78 \%$ in this conformation vs. $64 \%$ once $\mathrm{Na}^{+}$was bound (Table 4.2). Addition of $\mathrm{Na}^{+} /$Asp shifted the protein back toward the inward facing conformation, increasing this population to $84 \%$ (Table 4.2). DL-TBOA shifted the distribution to the longer distance corresponding to the outward facing conformation, trapping the protein (Figure 4.3d, Table 4.2). Adding 30\% sucrose shifted the $\mathrm{Na}^{+}$and $\mathrm{Na}^{+} /$Asp bound states back toward the inward facing conformation with increases to $81 \%$ and $100 \%$, respectively (Table 4.2). However, sucrose did not affect conformational populations in the substratefree state (Table 4.2). This implies either that the outward and inward facing conformations are equally solvent accessible in the substrate free form, or that the 
conformations are not in equilibrium, and the energy barrier between the outward and inward facing conformations is high in the substrate free state.

Table 4.2. Conformational populations based on distance distributions at

T375R1. The populations were calculated based on the area under each peak in Long Distances

\begin{tabular}{c|c|c} 
& Population 1 (\%) & Population 2 (\%) \\
\hline Apo & $78 \pm 3$ & $22 \pm 3$ \\
$+30 \%$ sucrose & $77 \pm 3$ & $23 \pm 3$ \\
\hline Na $^{+}$ & $64 \pm 6$ & $36 \pm 5$ \\
$+30 \%$ sucrose & $81 \pm 3$ & $19 \pm 2$ \\
\hline Na $^{+}$/Asp & $84 \pm 4$ & $16 \pm 3$ \\
$+30 \%$ sucrose & $100 \pm 2$ & 0 \\
\hline DL-TBOA & 0 & $100 \pm 1$ \\
$+30 \%$ sucrose & 0 & $100 \pm 1$ \\
\hline
\end{tabular}

A rotamer library analysis of substrate free and $\mathrm{Na}^{+}$crystal structures in the inward facing conformation did not fully describe the distributions (Figure $4.4 \mathrm{a}, \mathrm{b}$ ). The experimental distributions were broader than the simulated distributions, suggesting the existance of unresolved conformations in both states (Figure $4.4 \mathrm{a}, \mathrm{b}$ ). In the crystal structure of the asymmetric transporter one protomer is seen in the outward facing state and two protomers are in the inward facing state (Figure 4.5b). The distribution generated by rotamer library analysis had an average distance that agreed better with both the substrate free and $\mathrm{Na}^{+}$distributions, suggesting that the majority of protein is present in an asymmetric conformation (Figure 4.5 a). This was expected since previous reasearch has shown that protomers transport independently (Erkins et al., 2013). 

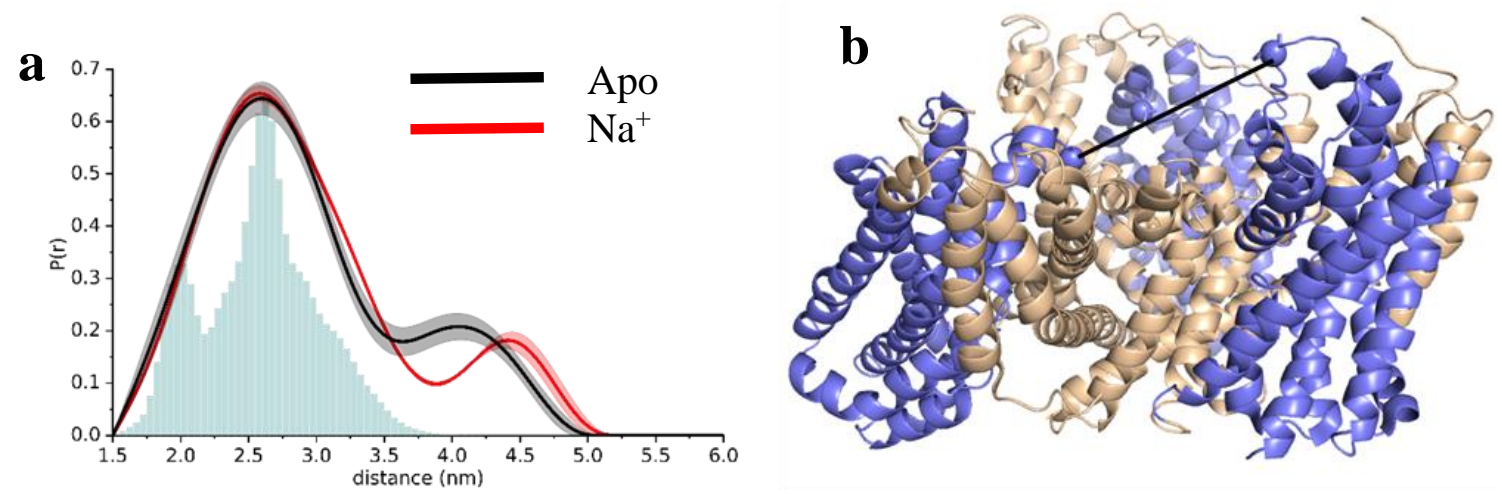

Figure 4.5. Substrate free and $\mathrm{Na}^{+}$bound distance distributions for T375R1 compared to rotamer library analysis of the asymmetric crystal structure (grey). a) DEER distance distributions compared to rotamer library analysis for the asymmetric crystal structure (3V8G). b) the asymmetric crystal structure with a line drawn between $\mathrm{T} 375$ in adjacent protomers to show the downward movement that occurs in the inward facing conformation.

\subsection{The conformationally restricted spin label, $R 1 p$, stabilizes the inward facing conformation}

The spin label 3-Methanesulfonilthiomethyl-4-(pyridin-3-yl)-2,2,5,5-tetramethyl2,5- dihydro-1H-pyrrol-1-yloxyl, designated $\mathrm{R} 1 \mathrm{p}$, is restricted to one rotameric state and can be used to determine the rotameric contributions made by $\mathrm{R} 1$ to distance distributions (Fawzi et al., 2011). If the width of the R1 distributions is due to rotameric exchange and not conformational exchange, the width of the peaks with the R1p spin label would be approximately $3 \AA$ A. However, if the width of the $\mathrm{R} 1$ peaks is due to conformational exchange, the distributions with the R1p spin label would still be broad. Distances at T375R1p were shorter than those using R1, with an average distance of 20-21 $\mathrm{A}$ (Table 4.1). The peak width was also narrower at 6-8 $\AA$, which indicated some conformational heterogeneity (Table 4.1). However, the conformational equilibria were shifted toward the inward facing conformation unless inhibitor was bound, which locked it in the outward facing conformation (Figure $4.6 \mathrm{~d}$ ). 

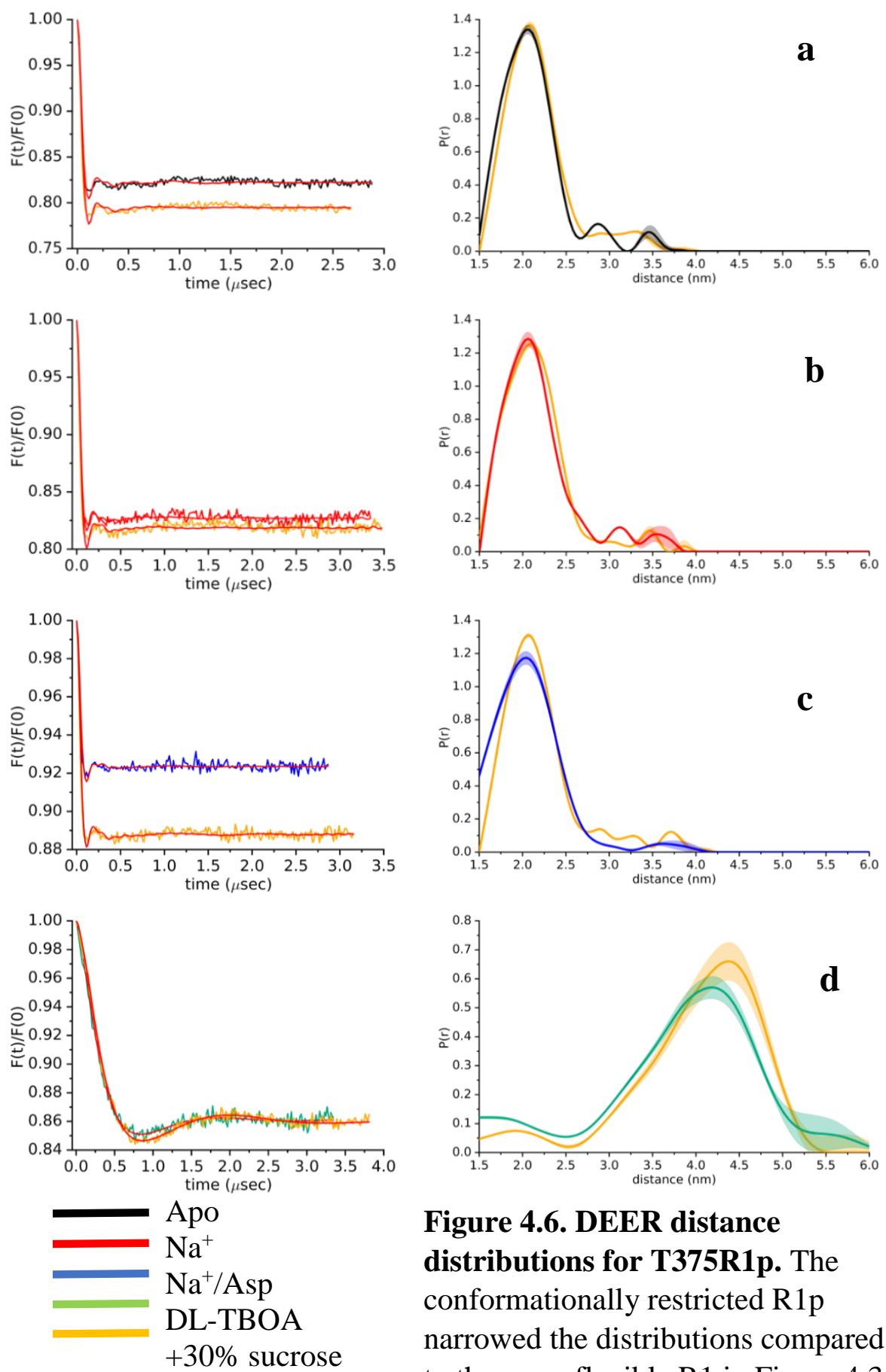

Figure 4.6. DEER distance

distributions for T375R1p. The conformationally restricted $\mathrm{R} 1 \mathrm{p}$ narrowed the distributions compared to the more flexible R1 in Figure 4.3. Distances were obtained by Tikhonov regularization, and substrate states are detailed by the legend. 
Adding 30\% sucrose did not alter conformational populations because it was already primarily sampling the inward facing conformation (Figure $4.6 \mathrm{a}, \mathrm{b}, \mathrm{c}$ ). This was not expected for a site that was aqueous exposed and not coming into tertiary contacts based on the crystal structures.

Since T375 was expected to be aqueous exposed, the CW-EPR spectra would reflect whether this was the true local environment of the spin label. The spectra would be mobile and reflect the spectra for an aqueous exposed loop site (Figure 3.6). However, the spectra were complex, multicomponent spectra that showed tertiary contacts (Figure 4.7). When compared to the substrate free protein, mobility increased when inhibitor was bound, reflecting a larger change in the local environment than for $\mathrm{Na}^{+}$or $\mathrm{Na}^{+} / \mathrm{Asp}$ (Figure 4.7). However, sucrose modulated the substrate free state, which did not agree with the distance distributions that showed no sucrose modulation in this state (Figure 4.7, Figure 4.4 a). Although the transport domain is locked in the outward facing conformation when inhibitor is bound, sucrose still modulated the local environment, further establishing conformational heterogenity within the outward facing conformation (Figure 4.7). This could be the conformation that was being modulated in the R1 CW spectra of the substrate free state. The CW spectra for R1p were broad as expected for a rotamerically restricted label (Figure 4.7). Similar to the R1 spectra, the lineshape was broadened slightly by substrate, and mobility was increased with inhibitor (Figure 4.7). Sucrose also broadened the spectra in all substrate states, which indicated that the label was coming into stronger tertiary contacts with the local environment, even in the inhibitor bound state. These spectra showed that T375 was not an aqueous exposed site that was not coming into contact with the local environment. However, these experiments 
did not distinguish whether the spin label is interacting with the bilayer, modulating protein energetics between conformations, or altering protein interactions with the bilayer.
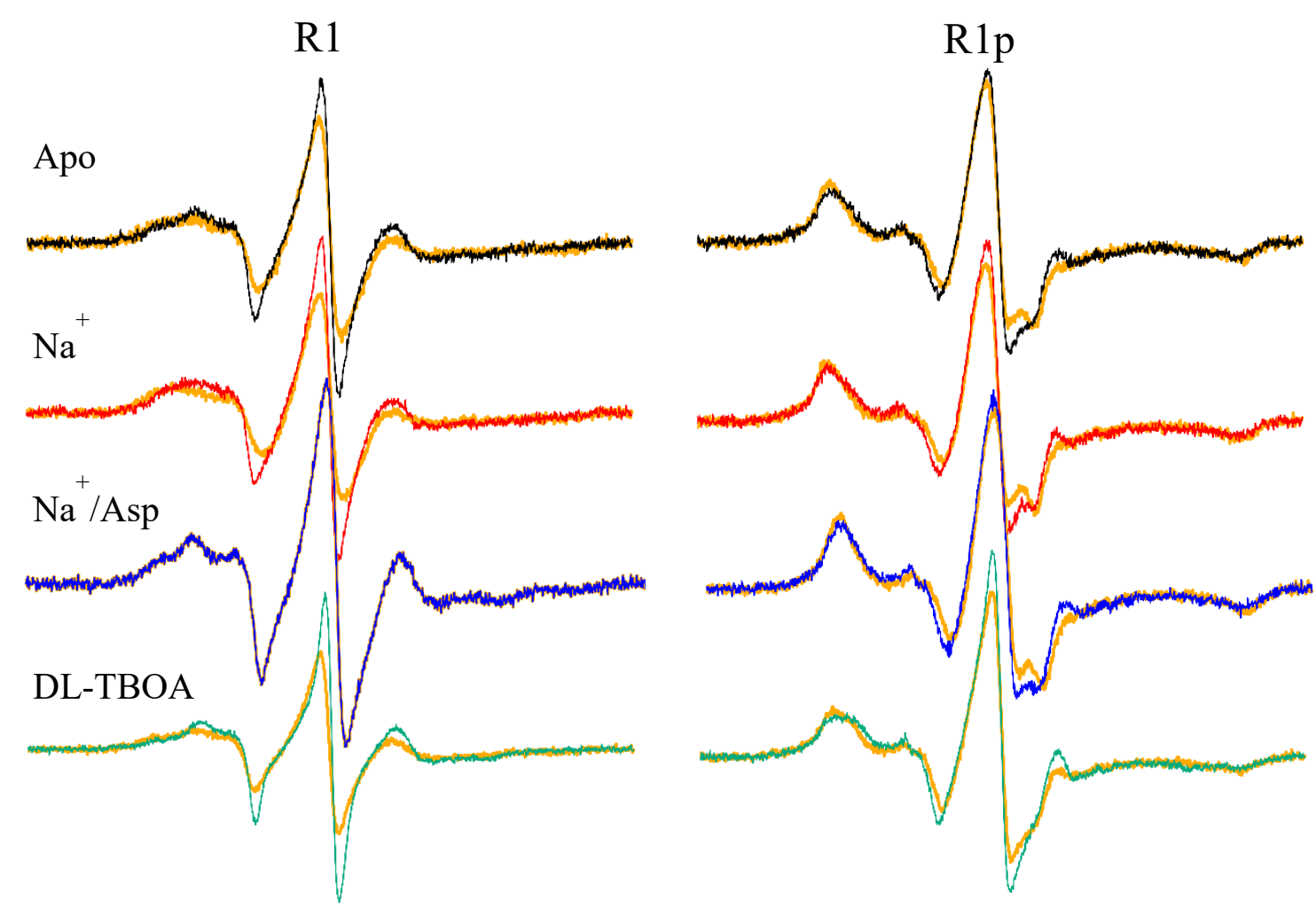

Figure 4.7. CW-EPR spectra for T375R1 and T375R1p. The R1 spectra indicated tertiary contacts, which were not expected based on the crystal structures, and the lineshapes were modulated by substrate, inhibitor, and sucrose. The R1p spectra were also multicomponent and were broadened by substrate and sucrose.

\subsection{Accessibility and depth measurements show unexpected protein/lipid headgroup contact}

Glt $t_{\mathrm{ph}}$ is typically modelled with a hydrophobic membrane thickness that matches the hydrophobic length of TM 1, leaving approximately $15 \AA$ A exposed on either side of the membrane (Yernool et al., 2004). However, recent coarse-grained and atomistic simulations show a greater membrane hydrophobic thickness than the length of TM 1 (Figure 4.8) (Stansfield et al., 2015). Power saturation EPR accessibility and depth 
measurements can determine the position of the spin label in relation to the membrane and allow mapping of the headgroup region (Lin et al., 1998). To determine whether the membrane or aqueous exposure at particular sites on Glt $\mathrm{ph}_{\mathrm{p}}$ matches the exposures predicted based upon the crystal structure and membrane models, accessibilities to NiEDDA and oxygen were measured at select sites in the 3-4 loop, transport domain, and trimerization domain. Accessibilities were measured with either $\mathrm{Na}^{+} / \mathrm{Asp}$ or DL-TBOA bound since $\mathrm{Na}^{+} /$Asp trapped the transport domain in the inward facing conformation with the spin label at T375, and DL-TBOA trapped the transport domain in the outward facing conformation.
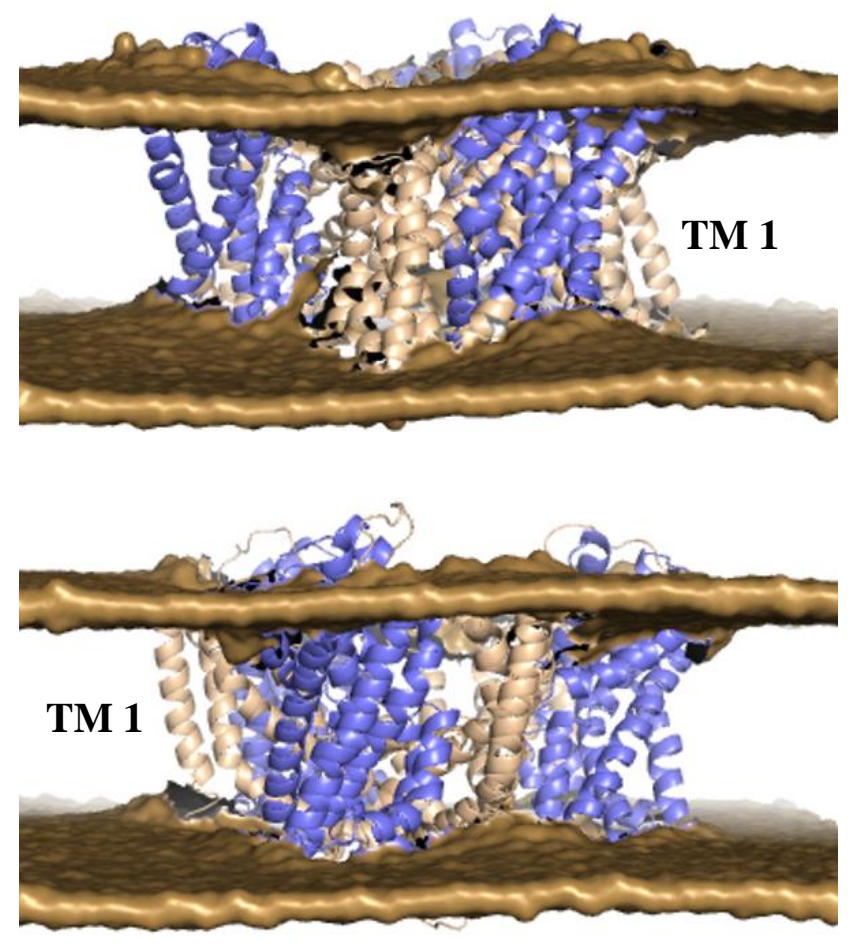

Figure 4.8. Final frame from atomistic simulations of Glt $_{\mathrm{ph}}$ with headgroup regions defined. The hydrophobic thickness is larger than the length of TM 1, which is typically how it is defined. (Adapted from MemProtMD). 
A membrane topology map based on newer models was used to predict aqueous or membrane contact (Figure 4.9). In the outward facing conformation, T375 and V176 were expected to be in the bulk aqueous. T166 was expected to be in contact with the lipid headgroups. Q121, K125, and P128 were difficult to predict based on the model since they were not well resolved in the crystal structure. In the inward facing conformation, T375 and V176 were predicted to be in contact with the lipid headgroups, which was not expected or predicted previously based on the crystal structures.

Hydrocarbon contact was predicted for T166, and lipid headgroup contact was predicted for Q121, K125, and P128, which were better resolved in this structure. However, since the 3-4 loop is a crystal contact site, this must be interpreted with caution.

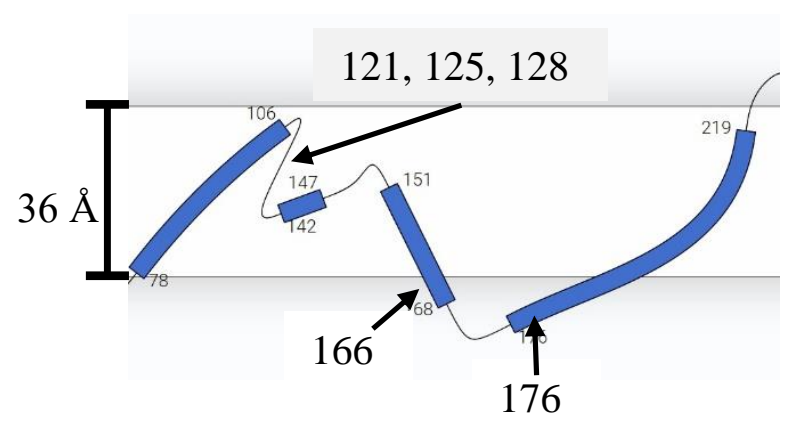

$121,125,128$

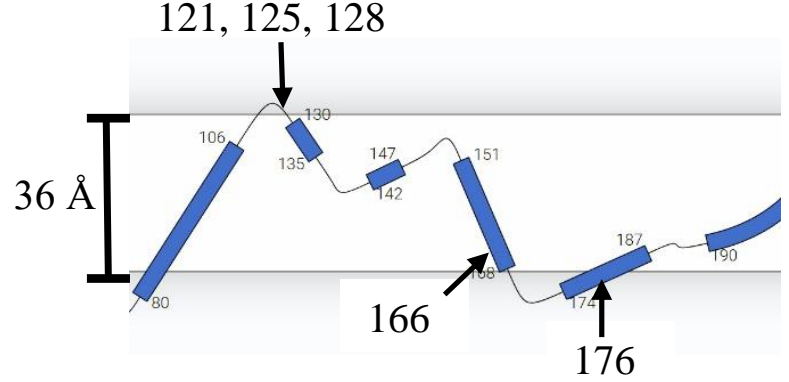

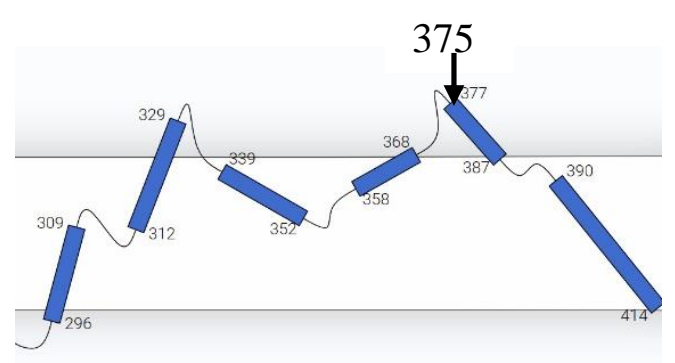

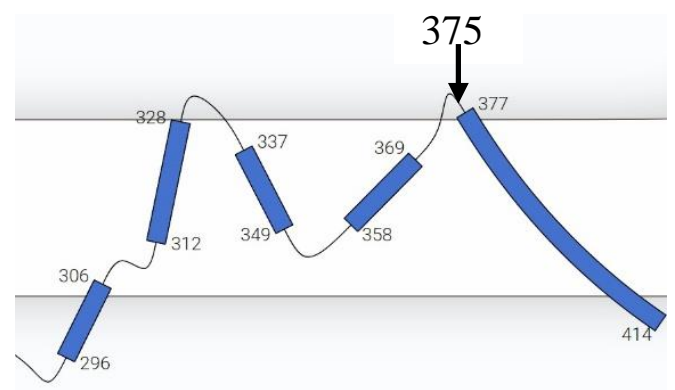

Figure 4.9. Membrane topology map for select GItph sites in the outward and inward facing conformations. Membrane contact was modelled in a DPPC membrane with a hydrophobic thickness of $36 \AA$ (white), and the horizontal lines denote the start of the headgroup region. The simulations were performed by MemProtMD. 
Depth measurements for membrane and aqueous controls calibrate the measurements to the lipid system. TEMPO-PC was the lipid headgroup control and was 6 A below the phosphate, which agreed with previous TEMPO-PC measurements (Dalton et al., 1987). The bulk aqueous label 3-carboxyproxyl measured $3 \AA$ above the phosphate. Depth measurements for T375R1 showed that the site was approximately $1 \AA$ below the phosphate when $\mathrm{Na}^{+} /$Asp was bound and between 0-2 $\AA$ above the phosphate in the presence of DL-TBOA (Figure 4.10). Due to the 2-3 $\AA$ error in typical power saturation measurements, it is possible that these are in the same environment. However, the significant increase in mobility in the CW spectra when DL-TBOA was bound compared to when $\mathrm{Na}^{+} /$Asp was bound suggest that these sites are actually in different environments (Figure 4.7).

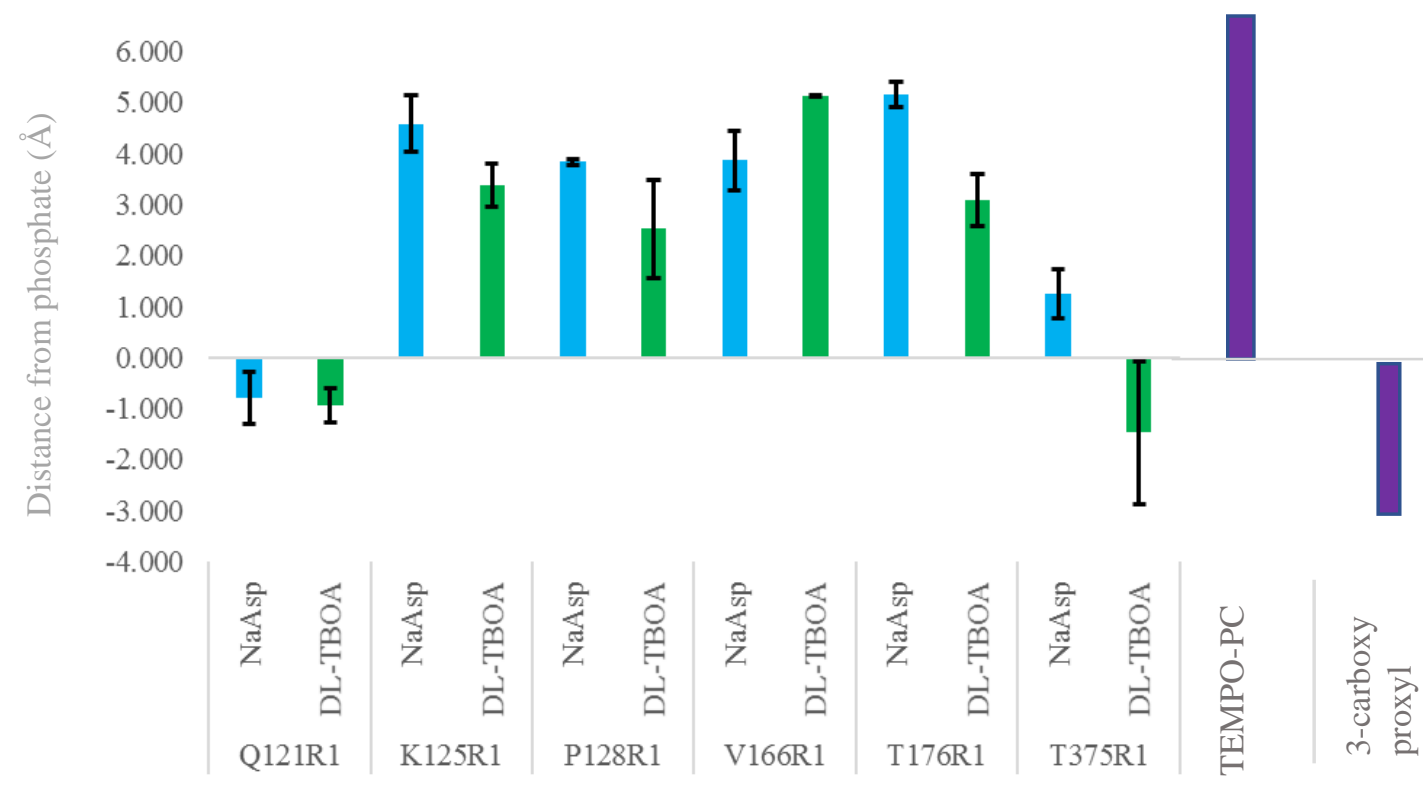

Figure 4.10. Depth measurements for select sites in the 3-4 loop, transport domain, and trimerization domain. Zero $\AA$ is at the phosphate group. Positive values are below the phosphate, and negative values are above the phosphate in the aqueous phase. 
It is also possible that the conformational heterogeneity in the outward facing conformation contributed to the large range of depths, and this site is actually sampling a range of distances in relation to the lipid headgroups. However, measurements in the headgroup region agreed with the membrane topology model.

Q121R1 measured approximately 1-2 $\AA$ above the phosphate, while K125R1 and P128R1 were 4-5 A below the phosphate. Since the 3-4 loop has been shown to be functionally important (Compton et al., 2010), membrane contact for some portion of the loop was not surprising. These measurements also agreed well with the $\mathrm{CW}$ spectra for these sites. The $\mathrm{Na}^{+} / \mathrm{Asp}$ and DL-TBOA bound CW spectra for Q121R1 were both indicative of a solvent exposed loop site (Figure 4.11). The CW spectra for K125R1 and P128R1 both showed multiple components and tertiary contacts, which would be consistent with either protein or headgroup contact (Figure 4.11).

Depth measurements for T166R1 and V176R1 placed them both in the headgroup region, approximately $5 \AA$ below the phosphate, when $\mathrm{Na}^{+} /$Asp was bound (Figure 4.10). This agreed with the membrane topology model (Figure 4.9).

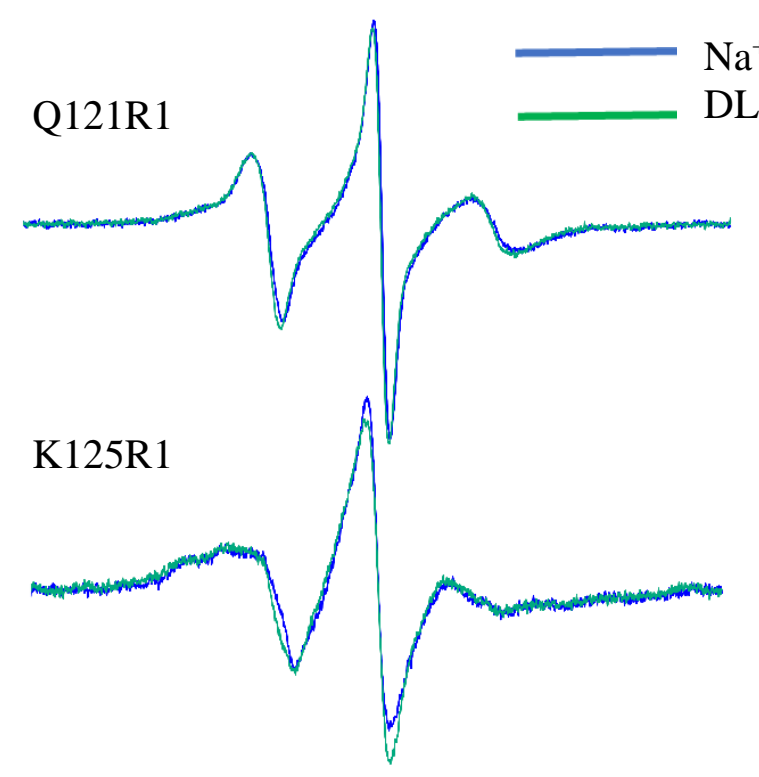

Figure 4.11. CW-EPR spectra of Q121R1, K125R1, and P128R1. The lineshape changes between the $\mathrm{Na}^{+} /$Asp and DL-TBOA bound states were negligible. 
When DL-TBOA was bound, locking the transporter in the outward facing conformation, V176R1 moved toward the aqueous phase (Figure 4.10), which was indicated by the model (Figure 4.9) and expected based on the crystal structures.

The accessibility measurements can determine if a site is in contact with the lipid or the protein interior. At both T166R1 and V176R1, NiEDDA and $\mathrm{O}_{2}$ accessibilities were both low, indicating that the side chain was protected and possibly facing the protein interior (Figure 4.12). The tertiary contacts in the CW spectra for T166R1 agreed with this (Figure 4.13). However, the CW spectra for V176R1 did not, as they were more mobile in comparison (Figure 4.13).

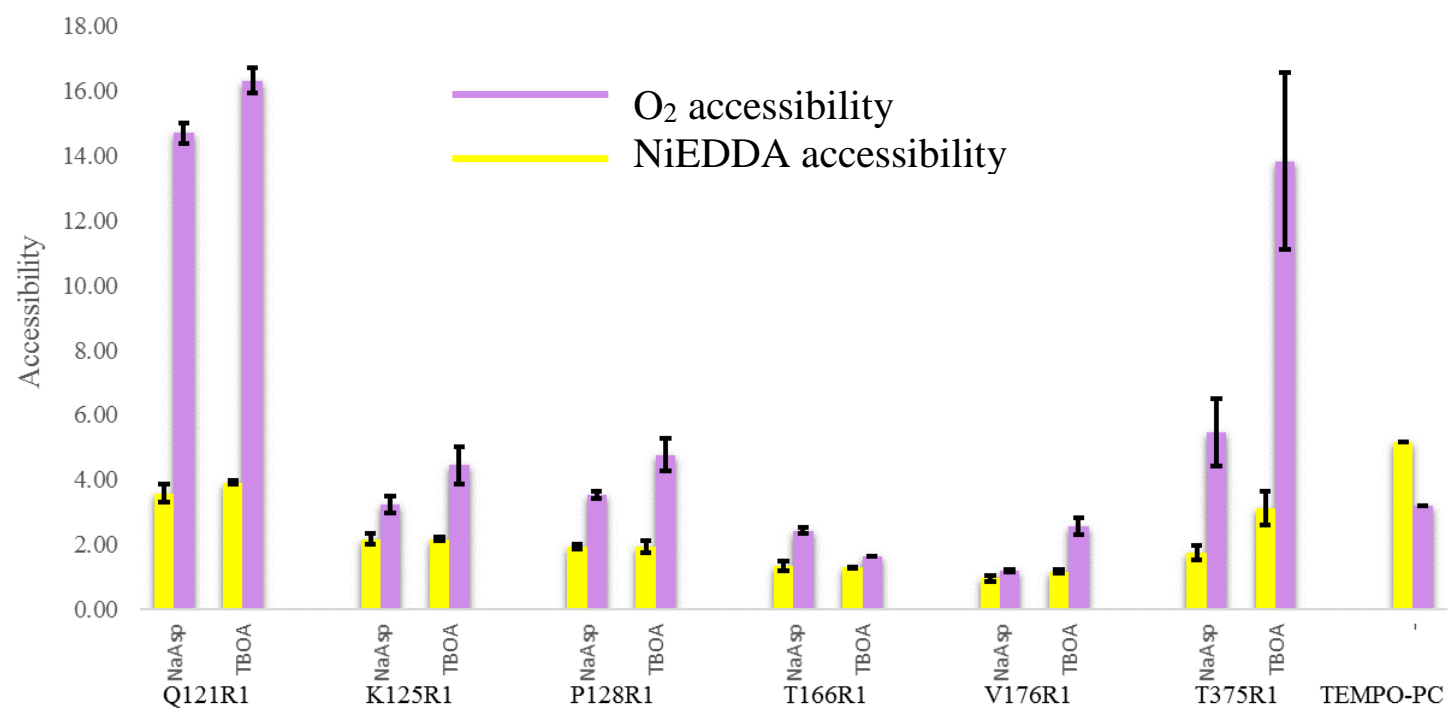

Figure 4.12. $\mathrm{O}_{2}$ and NiEDDA accessibility measurements for select sites in Glt $_{\mathrm{ph}}$. High NiEDDA accessibility denotes an aqueous facing residue, while high $\mathrm{O}_{2}$ accessibility denotes a membrane contact residue. If both accessibilities are low, this suggests a site protected by the protein interior. TEMPO-PC was used as the headgroup control and was positioned $6 \AA$ A below the phosphate. 


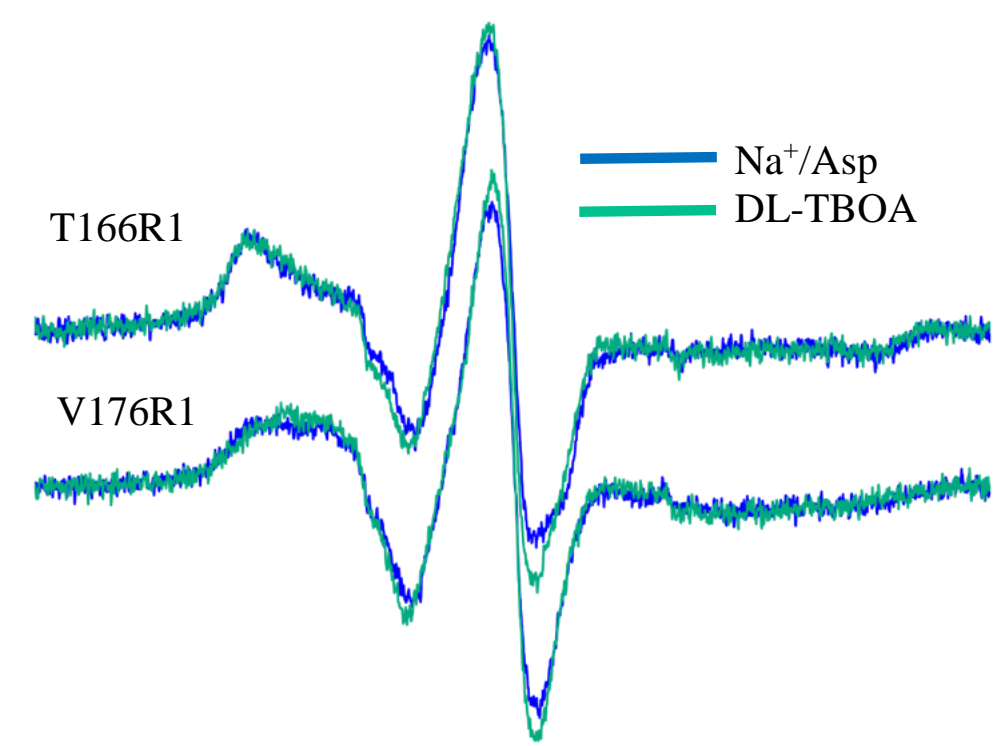

Figure 4.13. CW-EPR spectra of T166R1 and

V176R1. Both showed small mobility increases in the DL-TBOA bound state.

K125R1 and P128R1 also could have been contacting the protein interior. However, the increased accessibility to $\mathrm{O}_{2}$ and NiEDDA at these sites suggested that they are closer to the headgroups and not being protected by the protein interior (Figure 4.12). T375R1 could either be at the phosphate or near the bulk aqueous when inhibitor is bound. The accessibility measurements suggested that bulk aqueous contact was more likely as the NiEDDA accessibility was much higher when inhibitor was bound compared to $\mathrm{Na}^{+} / \mathrm{Asp}$ (Figure 4.12).

\subsection{Lipid headgroups modulate intermediate or asymmetric conformations}

Previous research has shown that decreasing lipid headgroup methylation

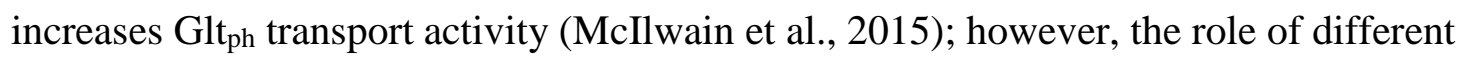
lipids in the transport process has not been described. CW-EPR spectra and DEER distance distributions were recorded in either 3:1 E. coli polar lipids:POPE (unmethylated) or E. coli polar lipids:POPC (3 methyl groups) to see any differences in 
conformational exchange in the different lipid environments. Distance distributions and CW-EPR spectra for T375R1 were also recorded in 3:1:1 DOPE:DOPC:DOPG to determine if greater membrane fluidity affected sampled conformations. CW spectra were recorded for V166R1 and T176R1 in 3:1 E. coli polar lipids:POPE and 3:1 E. coli polar lipids:POPC to determine if the lipid headgroups modulated the trimerization domain. The identity of the lipids was not expected to have an effect at sites that are not undergoing conformational exchange.

Changes in the CW spectra at V176R1 in the trimerization domain were negligible (Figure 4.14). This was expected for a trimerization domain site that was not
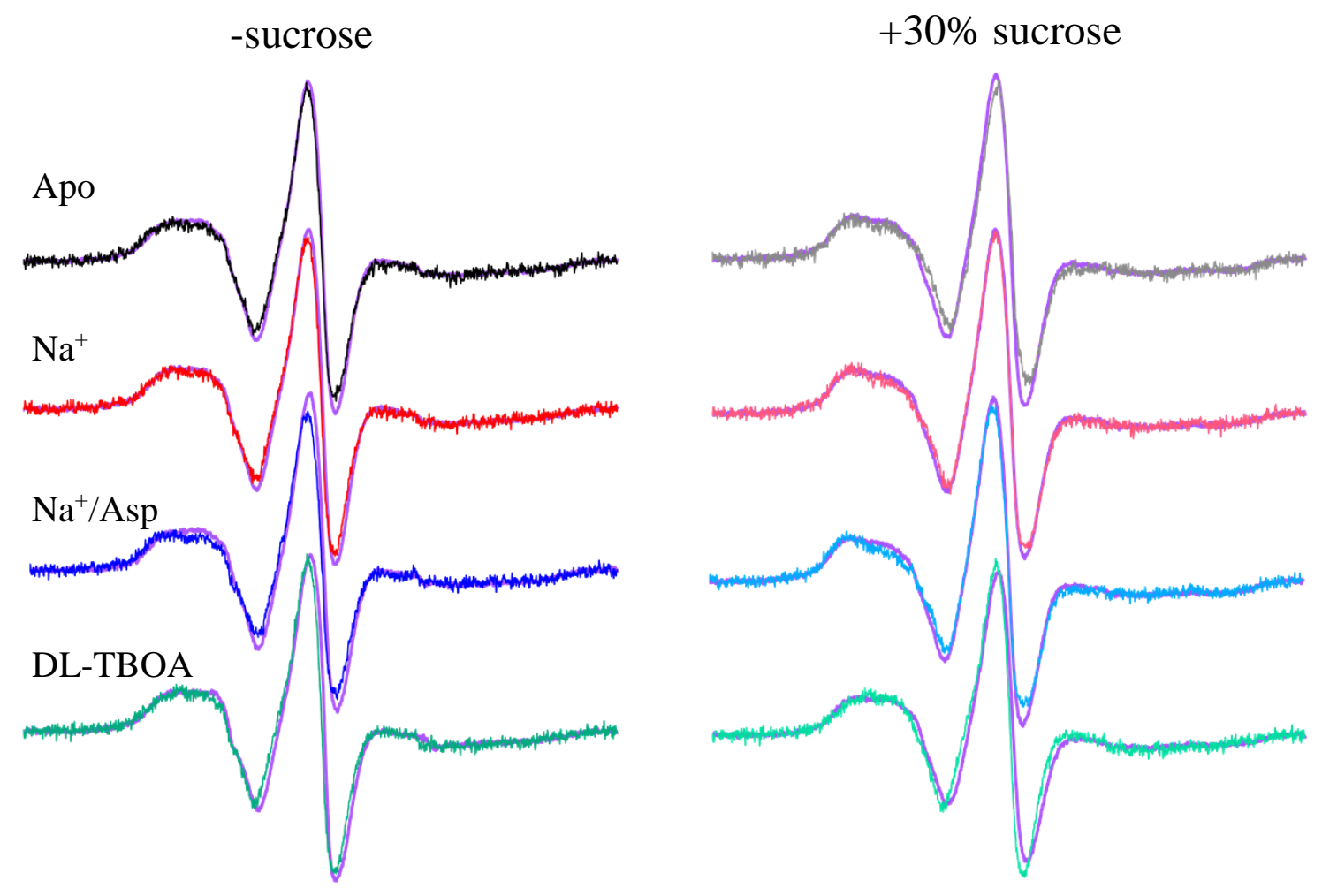

3:1 E. coli polar lipids:POPC

Figure 4.14. CW-EPR spectra of V176R1 in 3:1 E. coli polar lipids:POPE or 3:1 E. coli polar lipids:POPC. Lineshape changes were negligible between the two lipid systems. 
expected to be in contact with the lipid headgroups. This provided a control for transport domain sites or other sites that were expected to be in contact with lipid headgroups.

T166R1 showed larger mobility changes despite being in the trimerization domain. Mobility increased when POPC was substituted for the POPE when $\mathrm{Na}^{+}$or $\mathrm{Na}^{+} /$Asp was bound (Figure 4.15). The mobility increases were more apparent in the center line than in either the low field or high field lines, so these changes should be interpreted with caution. However, since the mobility increases were substrate dependent, they most likely indicate a change in the local environment around T166R 1 when $\mathrm{Na}^{+}$in bound to the protein.

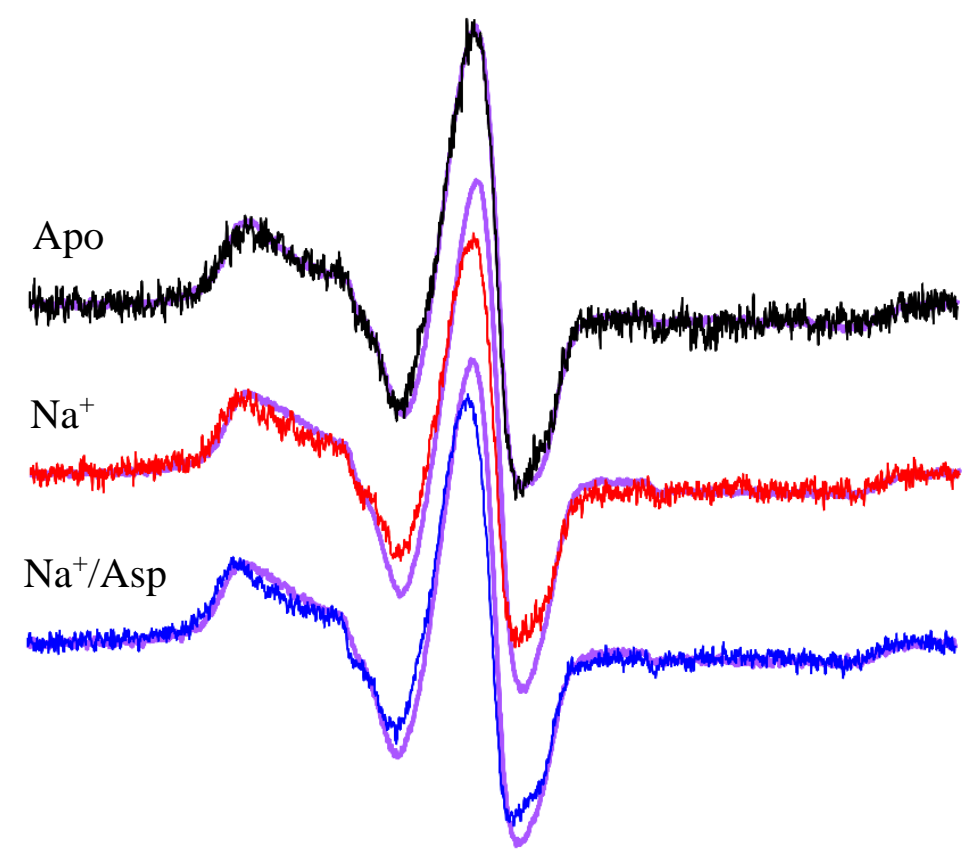

3:1 E. coli polar lipids:POPC

Figure 4.15. CW-EPR spectra of T166R1 in 3:1 $E$. coli polar lipids:POPE or $E$. coli polar lipids:POPC. 
Since T375 has been shown to be a good reporter of conformational changes, DEER distance distributions were recorded for the different lipid systems. The different lipids changed the DEER distributions for the substrate free and $\mathrm{Na}^{+}$bound states but not for the $\mathrm{Na}^{+} /$Asp bound state (Figure $4.16 \mathrm{a}, \mathrm{b}, \mathrm{c}$ ). The substrate free distribution in 3:1 $\mathrm{E}$. coli polar lipids:POPC showed an intermediate distance that was not resolved in the other distributions (Figure 4.16 a). Both peaks shifted approximately $2 \AA$ shorter in POPC for the $\mathrm{Na}^{+}$bound state (Figure 4.16 a). While that small of a shift was within error, there were no changes in the $\mathrm{Na}^{+} /$Asp bound state (Figure $4.16 \mathrm{c}$ ), so the changes seen in the substrate free and $\mathrm{Na}^{+}$bound states were most likely representative of changes in conformational populations. Conformational populations were also altered for the substrate free and $\mathrm{Na}^{+}$bound states when $30 \%$ sucrose was added (Figure 4.17 a,b). Once again, there were no changes when $\mathrm{Na}^{+} / \mathrm{Asp}$ was bound, so the substrate free and $\mathrm{Na}^{+}$ changes were most likely significant (Figure 4.17 c). DL-TBOA distributions were significantly altered by the lipids with and without sucrose (Figure 4.16 d, Figure $4.17 \mathrm{~d}$ ). The shortest distance in those distributions was an artefact, but the intermediate distance was not. Overall, changes were larger when POPC was substituted for POPE than they were for the 3:1:1 DOPE:DOPC:DOPG system (Figure 4.16, Figure 4.17). Therefore, changes in the distributions were due to modulation by headgroups and not by cardiolipin. 

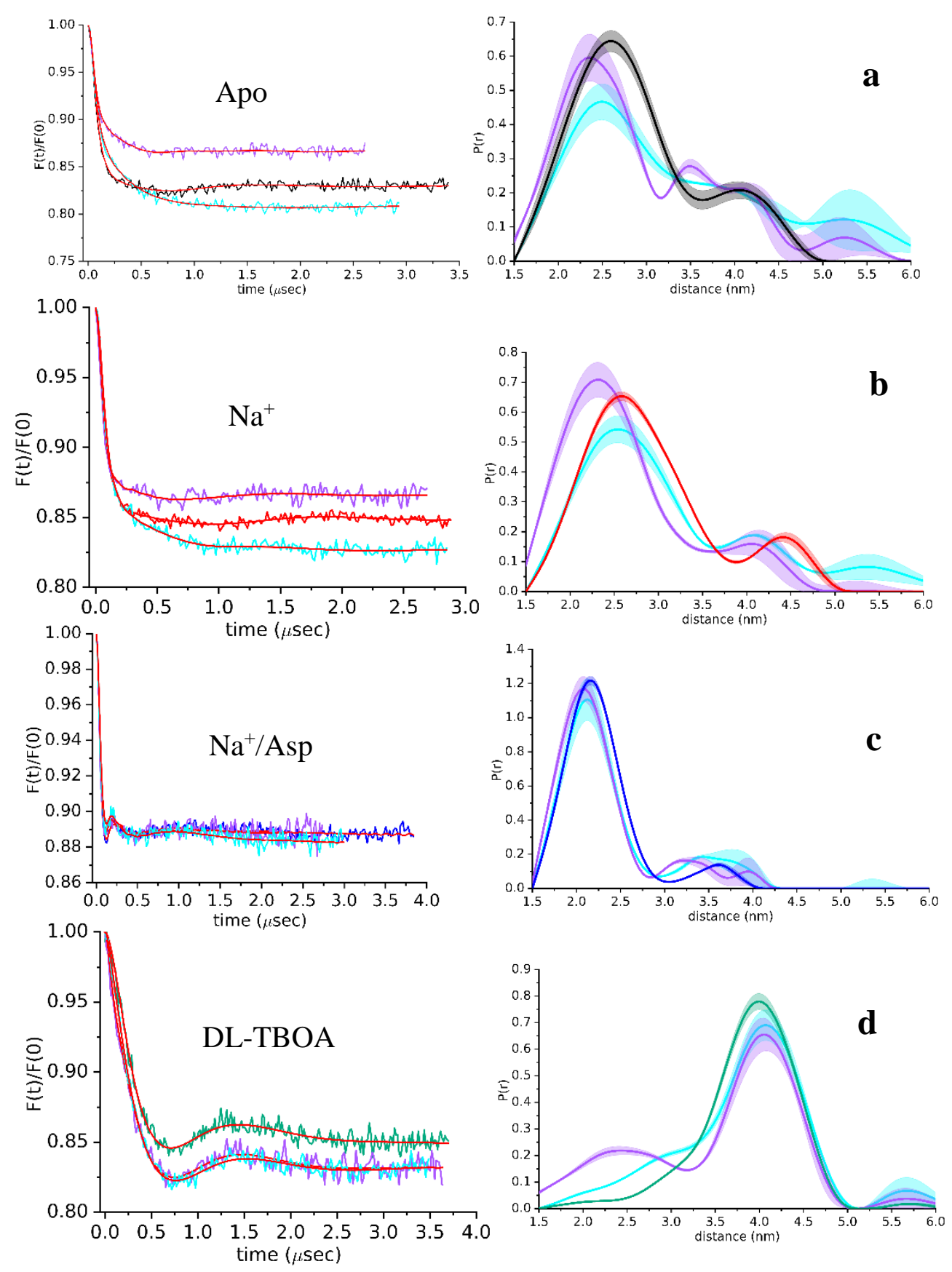

3:1 E. coli $\mathrm{PL}: \mathrm{POPC}$
3:1:1 DOPE:DOPC:DOPG

Figure 4.16. DEER distance distributions for T375R1 in different

lipid systems. Distances were obtained through Tikhonov regularization, and substrate states and liposome compositions are in the legend. 

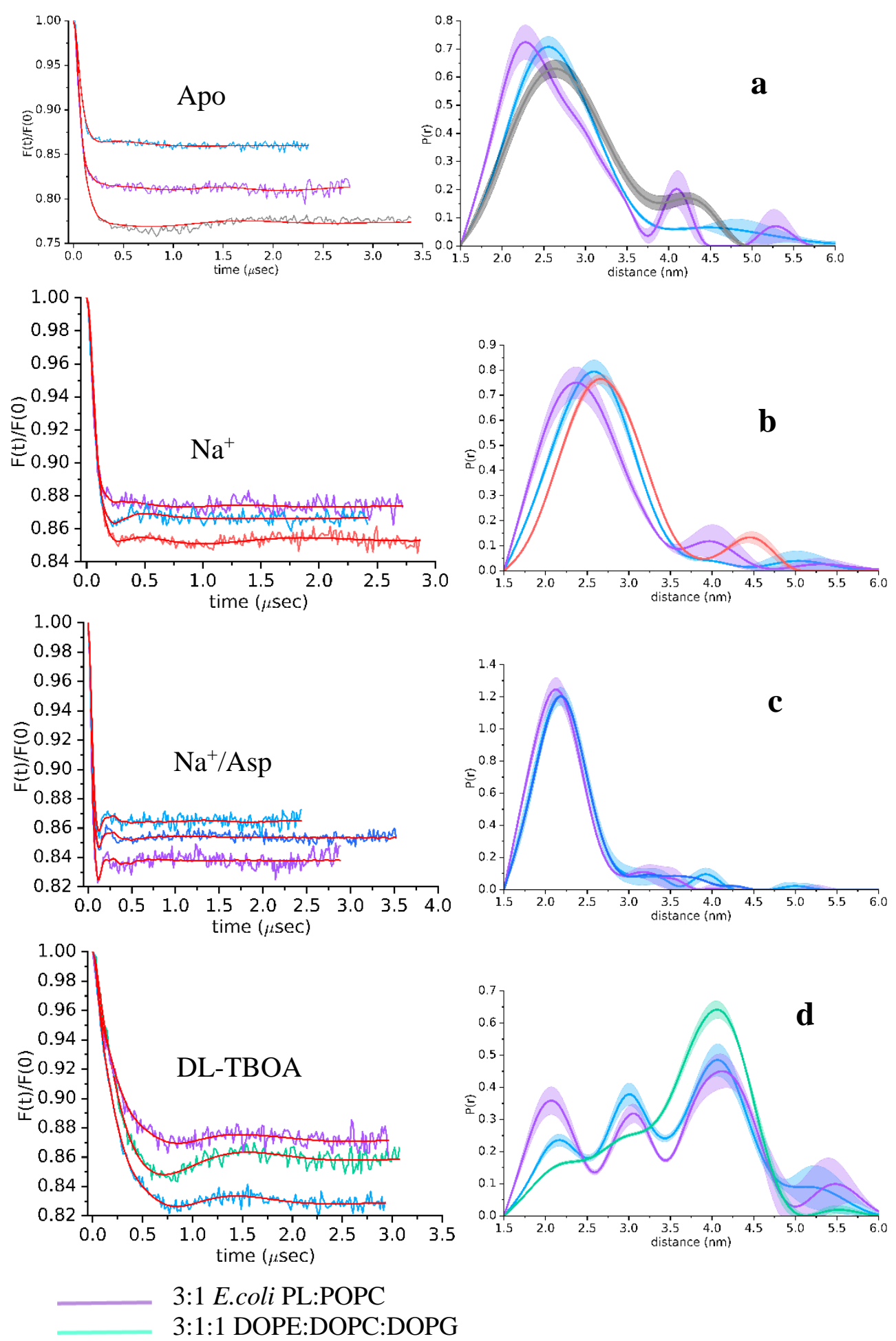

3:1:1 DOPE:DOPC:DOPG

Figure 4.17. DEER distance distributions for T375R1 in different lipid systems with $30 \%$ sucrose added. Distances were obtained through Tikhonov regularization, and substrate states and liposome compositions are in the legend. 


\subsection{The $\mathrm{R} 1$ side chain alters conformational populations in detergent micelles}

When Glt $\mathrm{ph}_{\mathrm{p}}$ is crystallized in detergent in the presence of the strong osmolyte polyethylene glycol 400 (PEG 400), the crystals only form with all three monomers facing outward. The protein must be crosslinked with lipids added to the crystallization buffer to capture the inward facing conformation (Verdon et al., 2014). Previous DEER studies of $\mathrm{Glt}_{\mathrm{ph}}$ in detergent micelles, however, have provided site specific results, with certain sites favoring either the outward or inward facing conformation (Figure A.1). T375R1 was shown to favor the inward facing conformation in the substrate free state and the outward facing conformation in the $\mathrm{Na}^{+} / \mathrm{Asp}$ bound state (Figure A.1). The stabilization of the outward facing conformation in protein crystals could be due to crystal contacts or the detergent micelle and protective osmolytes. DEER distance distributions were recorded for T375R1 in detergent micelles with and without the protective osmolyte sucrose to determine if the addition of the protective osmolyte stabilized the conformation seen in the crystal structures.

Distance distributions of T375R1 in detergent showed a shift toward the inward facing conformation in the substrate free and $\mathrm{Na}^{+}$bound states and a small shift toward the outward facing conformation when $\mathrm{Na}^{+} / \mathrm{Asp}$ was bound (Figure 4.18). Intermediate or asymmetric conformations appeared to be preferred to the completely outward facing conformation (Figure 4.18 a). While the peak around $52 \AA$ could correspond to the outward facing conformation if the spin labels were pointing away from each other, it was most likely an artefact due to background labeling of the detergent. The $\mathrm{CW}$ spectra showed significant background labeling that was only removed with a second size exclusion column (data not shown). 

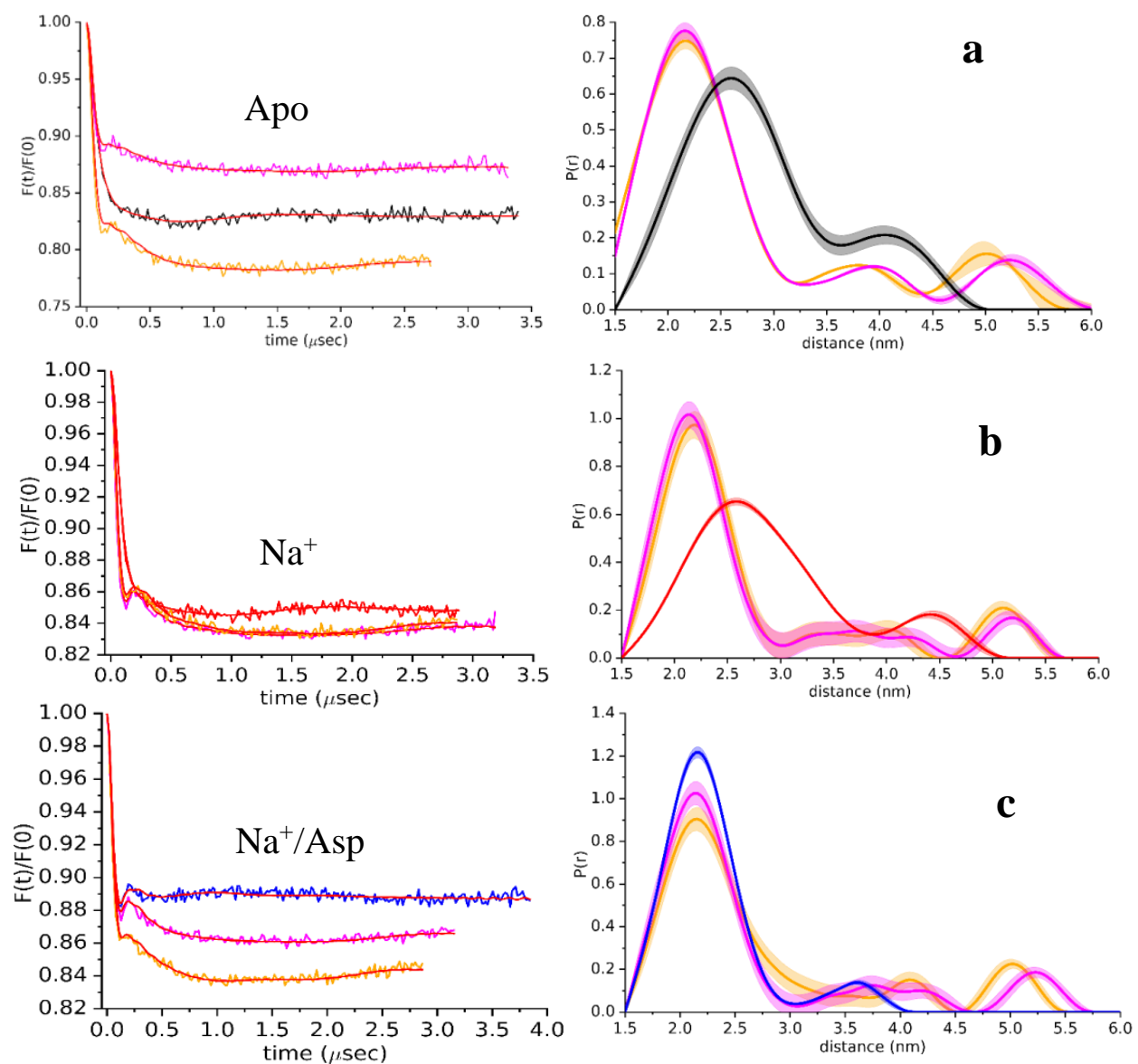

DDM

$\mathrm{DDM}+30 \%$ sucrose

Figure 4.18. DEER distance distributions for T375R1 in detergent micelles. Distances were obtained through Tikonov regularization, and substrate states are detailed in the legend; the black, red, and blue are in 3:1 E. coli polar lipid:POPE liposomes.

Sucrose did not shift populations in the substrate free state, which was similar to the result seen in liposomes (Figure 4.18 a). However, there was no a population shift in the $\mathrm{Na}^{+}$bound state, which contrasts with sucrose effect seen in lipids (Figure $4.18 \mathrm{~b}$ ). The distribution for the $\mathrm{Na}^{+} / \mathrm{Asp}$ bound state in detergent shifted toward the outward facing conformation when sucrose was added, which conflicted with the reconstituted system that showed increased stabilization of the inward facing conformation. (Figure 
$4.18 \mathrm{c})$. Since the previous DEER distributions showed site specific stabilization of the inward facing conformation, it was most likely the presence of the spin label altering conformational populations. This was also suggested by the stabilization of the inward facing conformation with the conformationally restricted R1p (Figure 4.6).

\subsection{HP1 samples conformations not observed in crystal structures}

Access to the substrate binding site in Glt $\mathrm{ph}_{\mathrm{ph}}$ is controlled by the two gates HP1 and HP2. The role of HP2 in extracellular gating has been described by the crystal structure of the inhibitor bound protein (Boudker et al., 2007). However, the role of HP1 in intracellular gating has been implicated by molecular dynamics simulations but has not been verified experimentally (Zomot and Bahar, 2013). Crystal structures show HP1 in two different environments.
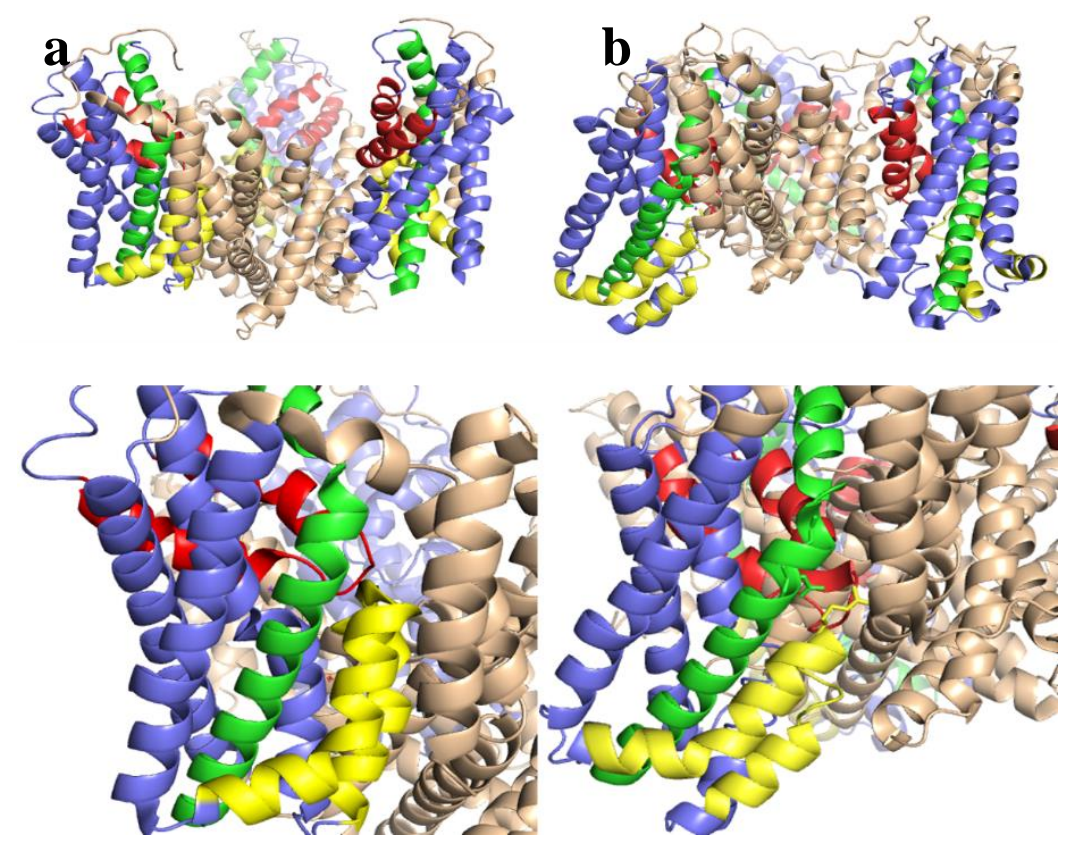

Figure 4.19. Crystal structures of Gitph in the outward (a) and inward (b) facing conformations with HP1 highlighted. The bottom images show the environment around HP1, which is yellow. HP2 is in red, and TM8 is in green. 
The environment around the apex of HP1 is crowded in the outward facing conformation with TMs 2, 7, and 8 within $7 \AA$ of the loop (Figure 4.19 a). However, in the inward facing conformation, HP1 interacts only with TM 8 and HP2. The environment is less crowded, which could potentially increase conformational heterogeneity (Figure 4.19 b).

The labelled sites at the apex of HP1 have been shown to be important to transport through mutagenesis studies and activity assays (Mindell et al., unpublished). R276 and S278 have both been shown to coordinate aspartate, so CW spectra were recorded for sites around these to avoid sites that were the most functionally important. The CW spectra showed at least two components, and the lineshapes were modulated by substrate (Figure 4.20). $\mathrm{Na}^{+} /$Asp addition increased mobility at all sites tested, suggesting

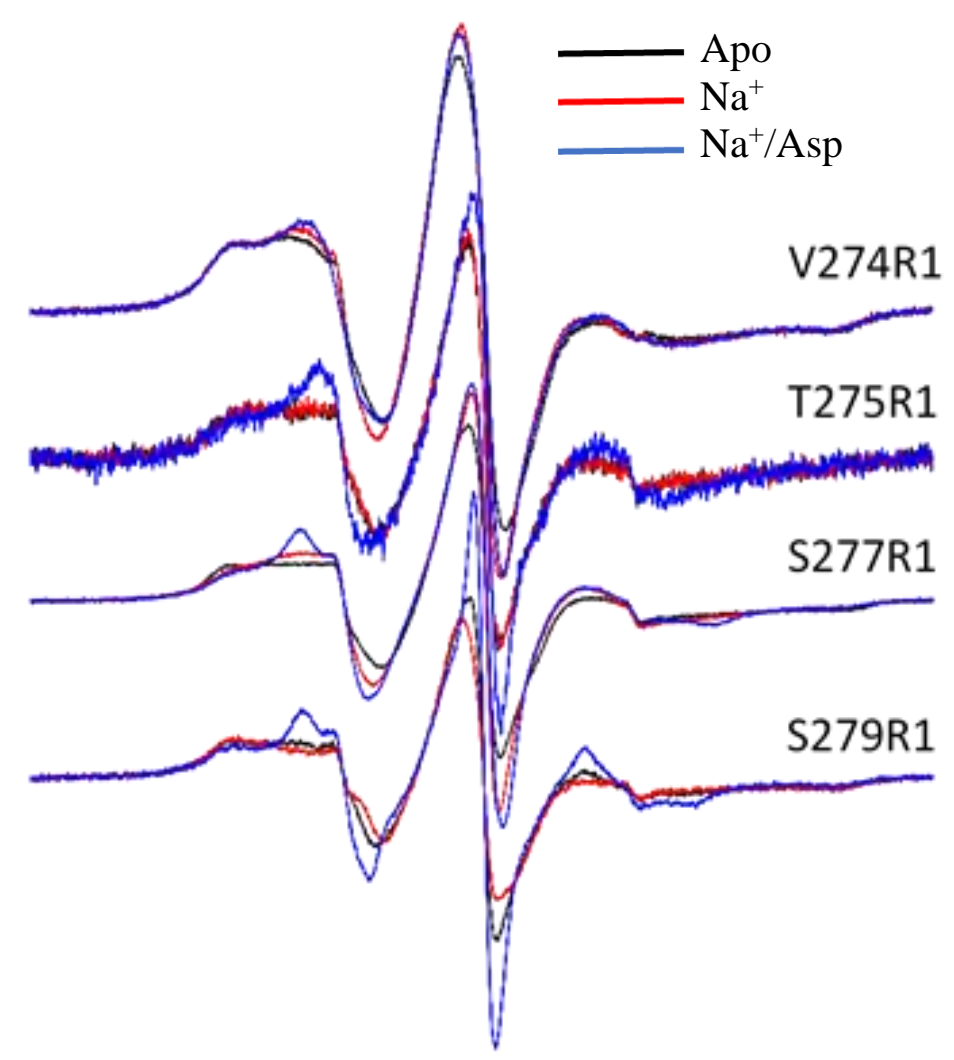

Figure 4.20. CW-EPR spectra at select sites adjacent to substrate coordinating residues in HP1. 
that substrate was able to bind properly in the substrate binding site with the spin label on a nearby residue and that a structural change is occuring that leads to less crowding.

Spectral broadening was observed for V274R1, S277R1, and S279R1 when 30\% sucrose was added and was larger for V274R1 and S277R1 in all three substrate states than for S279R1, which had the largest broadening in the $\mathrm{Na}^{+} / \mathrm{Asp}$ bound state (Figure

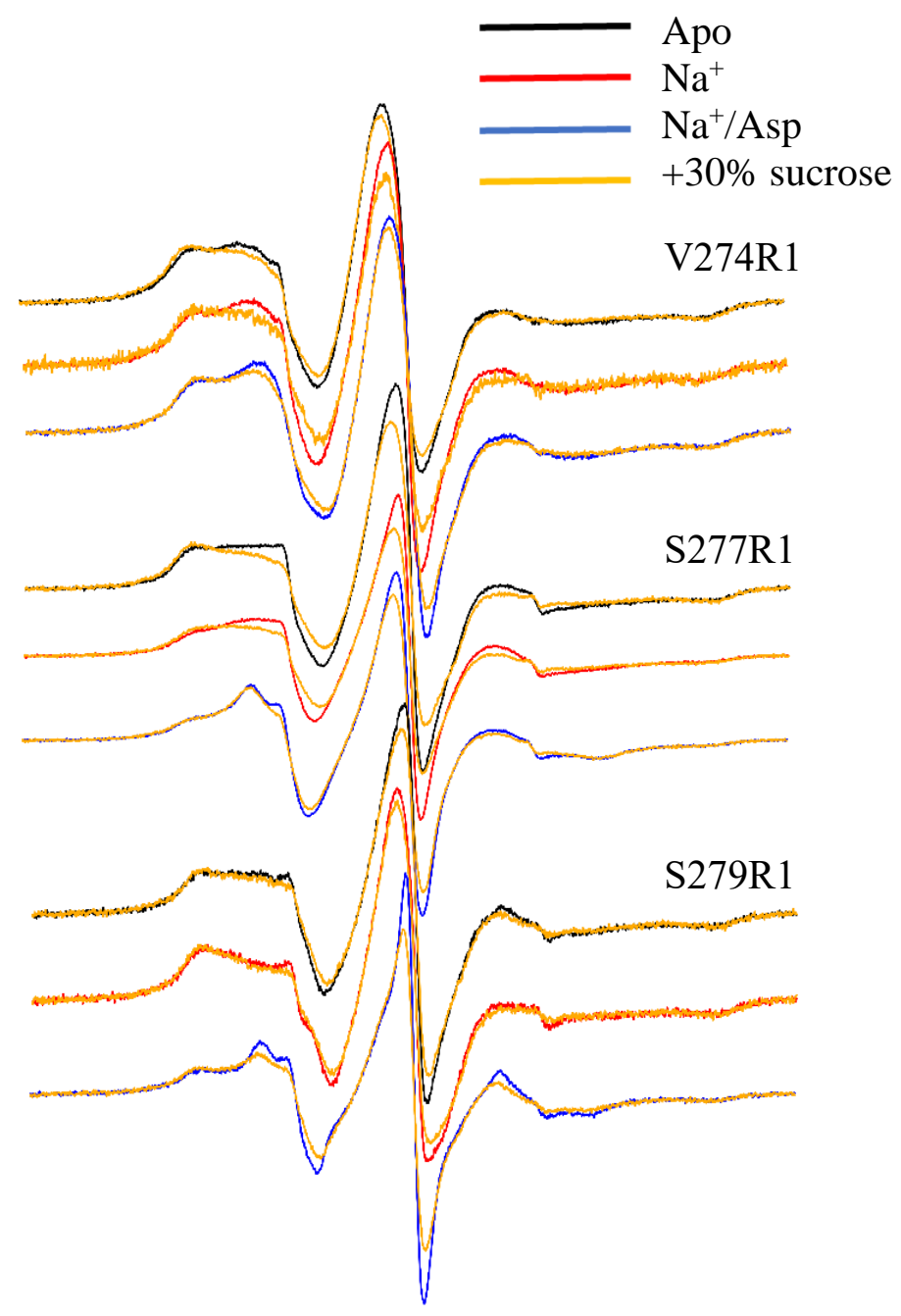

Figure 4.21. CW spectra for select sites at the apex of HP1 with and without $30 \%$ sucrose.

4.21). The broadening suggested a shift toward the outward facing conformation, which was in contrast to inward facing shift seen for T375R1 (Table 4.2). However, this could 
reflect shifts among conformations that do not directly correlate with the inward and outward facing conformations in the crystal structures. Therefore, it is possible that HP1 undergoes conformational exchange that is not reflected in the bulk of the transport domain.

S277R1 and S279R1 showed a third component of high mobility that represented approximately $1 \%$ of the total population as determined by spectral fitting (Figure 4.22). As these flank S278R1, which directly coordinates aspartate, the mobile component could correspond to a conformation that is important for substrate release that has not been captured by currently available crystal structures. The highest mobility component

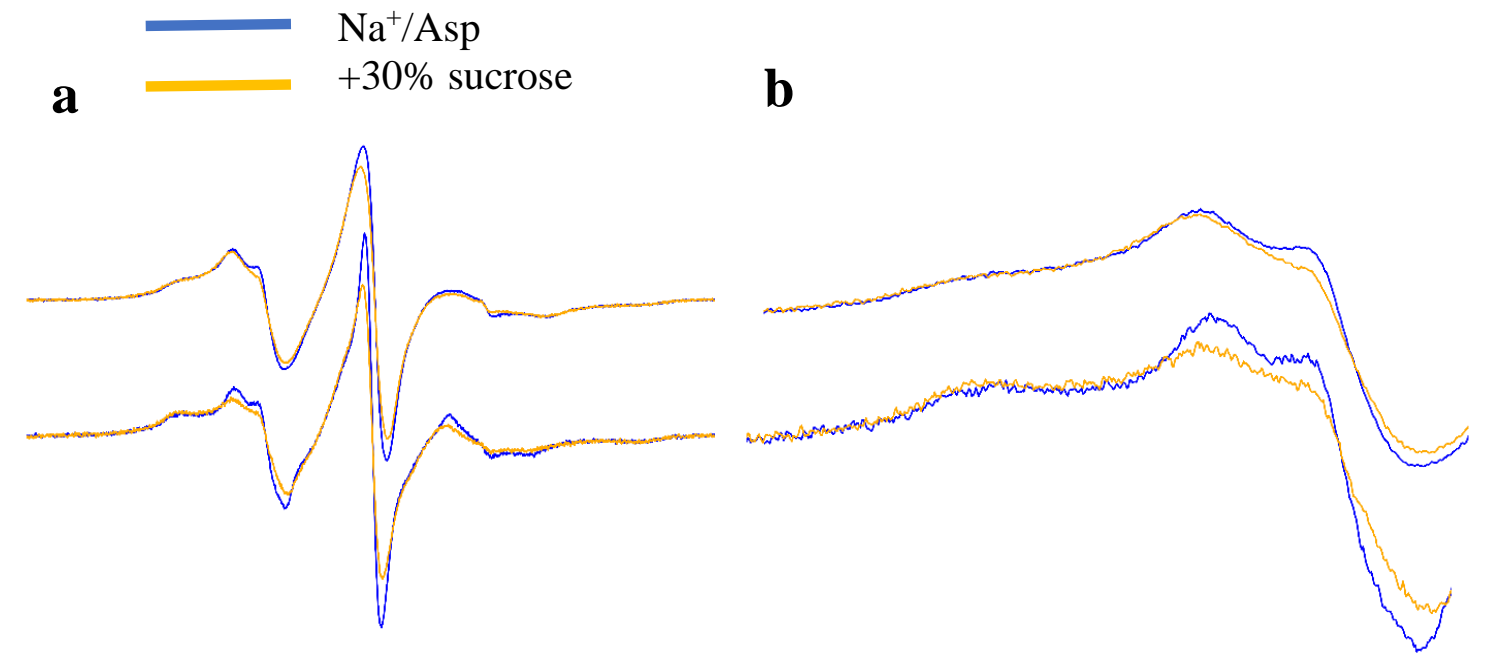

Figure 4.22. CW-EPR spectra of S277R1 and S279R1 with and without $30 \%$ sucrose. a) Full CW spectra. b) Zoom of the low field line showing conformational modulation by sucrose.

in $\mathrm{Na}^{+} /$Asp bound S277R1 and S279R1 was removed when 30\% sucrose was added, and the middle component in the low field line decreased as well (Figure $4.22 \mathrm{~b}$ ). The highest mobility component was not well represented by the crystal structures, which showed HP1 always in tertiary contact to at least TM 8. This component could represent an open 
conformation of HP1 that was able to release aspartate intracellularly (Figure 4.22 b), which would agree with the molecular dynamics simulations that showed increased conformational exchange of HP1 in the inward facing conformation (Zomot and Bahar, 2013). However, it is possible that these are two component spectra that are highly anisotropic. Regardless, Glt ph was most likely sampling a mixture of states throughout the transport domain that have not been not captured in the crystal structures.

\subsection{Chloride affects conformational population}

Glt $_{\text {ph }}$ has a thermodynamically uncoupled, substrate-gated chloride conductance that partially dissipates the membrane potential. Chloride conductance is not necessary for transport; however, transport rates were slowed when the nontransportable anion, gluconate, was substituted for the chloride (Ryan and Mindell, 2007). While the chloride conductance is known to partially dissipate the membrane potential, the underlying mechanism linking chloride conductance to transport rates is not fully understood. It is possible that either the chloride channel does not form properly when chloride is absent. It is also possible that there are currently unknown direct interactions of chloride with the transport domain. In either case the chloride conductance would be responsible for conformational exchange events during the transport cycle. As molecular dynamics studies have suggested that chloride conductance does not occur in the fully outward or fully inward facing conformations (Matchens et al., 2015), it is likely that it occurs during the high energy intermediates that can be seen as changes in the local environment in $\mathrm{CW}$ spectra, which were recorded with either chloride or the nontransportable anion gluconate. 
Mobility changes were negligible at T166R1, which would be expected for a trimerization domain site not part of the chloride ion channel (Figure 4.23). While there were small changes in the intensity of the center line, the changes were not seen throughout the spectra, so they were most likely not indicative of conformational exchange due to the gluconate substitution.

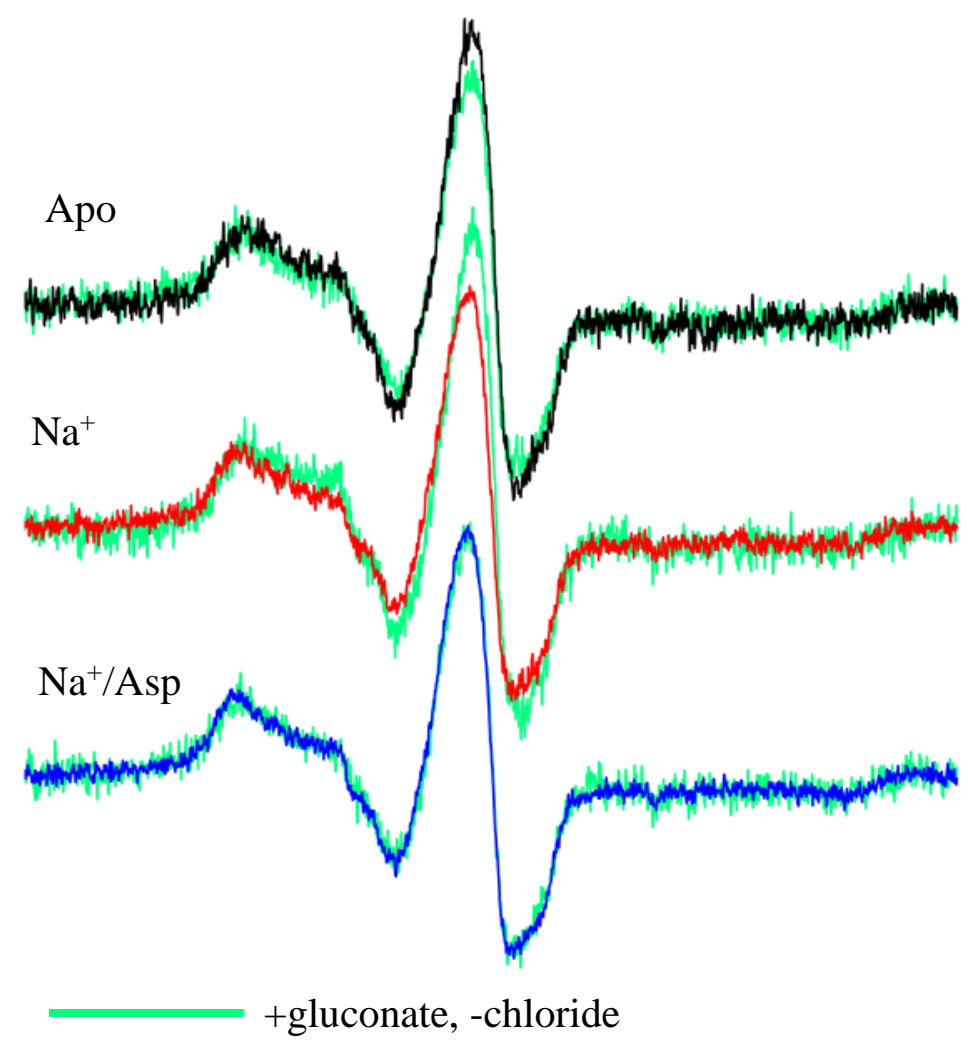

Figure 4.23. CW-EPR spectra for T166R1 with chloride or gluconate. All green spectra have gluconate substituted for chloride. Lineshape changes were negligible.

T375R1, while in the transport domain, is not part of the chloride ion channel. However, it does report on conformational exchange events, so structural changes that occur without chloride would be visible in the CW spectra. The CW spectra at T375R1 
showed substrate dependent changes in mobility when gluconate was substituted for the chloride. There were no lineshape changes observed for the substrate free and $\mathrm{Na}^{+}$bound states, which would be expected for substrate-gated chloride conductance (Figure 4.24). However, there were also no lineshape changes when $\mathrm{Na}^{+} / \mathrm{Asp}$ was bound until $30 \%$ sucrose was added, which broadened the spectrum (Figure 4.24). In contrast, mobility

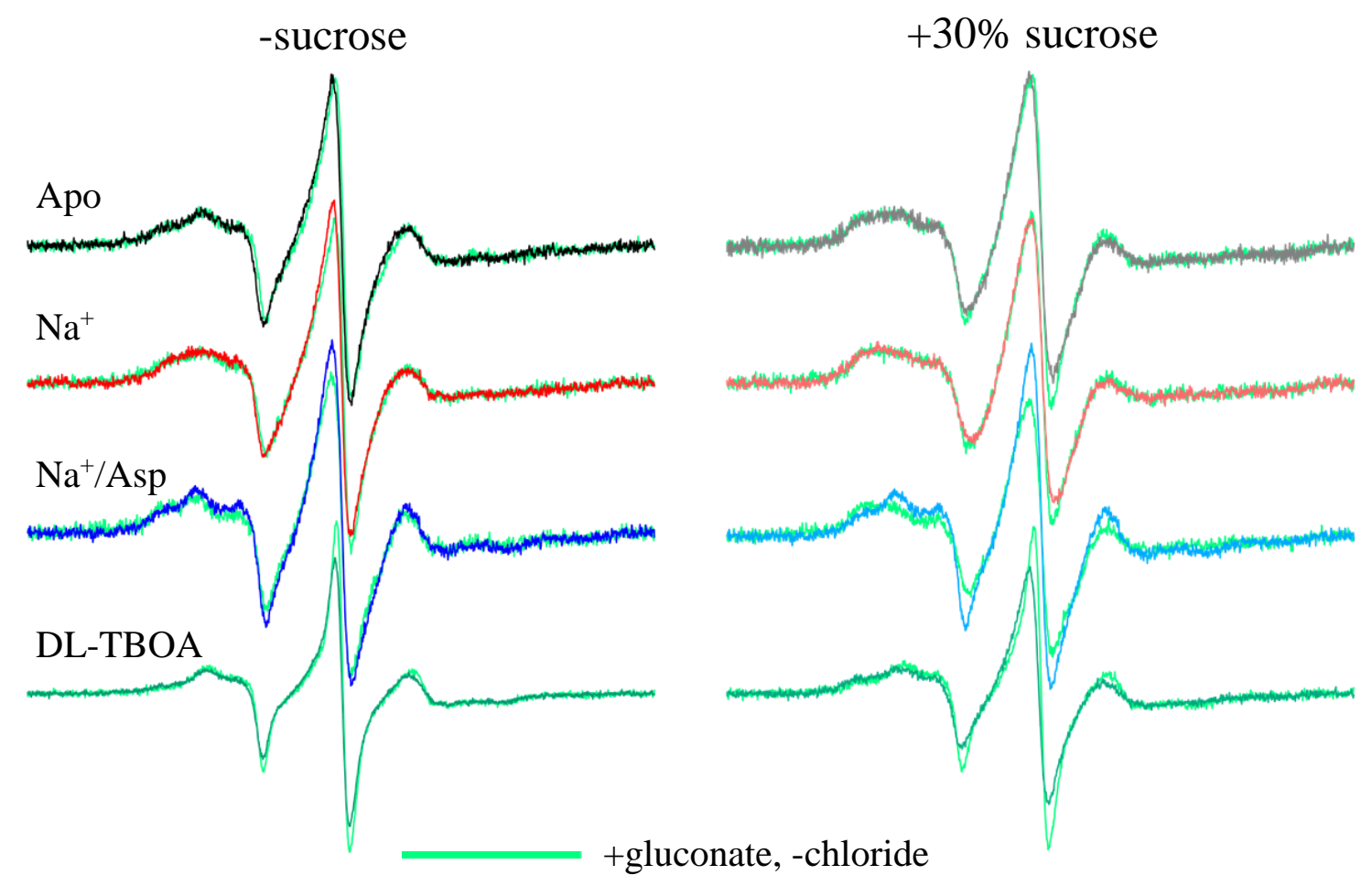

Figure 4.24. CW-EPR spectra for T375R1 with chloride or gluconate. Lineshape changes were substrate and inhibitor dependent, with broadening observed for $\mathrm{Na}^{+} /$Asp bound $+30 \%$ sucrose when gluconate was substituted. Gluconate substitution increased mobility when DL-TBOA was bound.

increased when DL-TBOA was bound, and the effect was enhanced by the addition of $30 \%$ sucrose (Figure 4.24). Since $\mathrm{Na}^{+} / \mathrm{Asp}$ and DL-TBOA trap the transport domain in the inward and outward facing conformations, respectively, when the spin label is attached at this site, this would contradict the MD results that suggested chloride 
conductance occurred during the intermediate states in the transport cycle. These spectra suggested that the extreme conformations of fully outward and fully inward were affected by the absence of chloride. It is possible that the channel forms upon substrate or inhibitor binding, while actual chloride movement occurs in the intermediate, so the channel may not be forming properly without chloride.

$\mathrm{K} 125 \mathrm{R} 1$ is in the 3-4 loop and is important for transport, although the exact role of the loop in structural changes during transport is not understood (Ryan and Mindell, 2007). Since it connects the transport and trimerization domains, this site could report on conformational changes necessary for chloride conductance. Mobility changes at this site with gluconate substitution were opposite those seen in the bulk of the transport domain with small mobility increases in the substrate free state but not in $\mathrm{Na}^{+}$or $\mathrm{Na}^{+} / \mathrm{Asp}$ bound states (Figure 4.25). Since this site appeared to report on $\mathrm{Na}^{+}$binding with an increase in mobility, the substitution of gluconate made the local environment of the substrate free state look more like the $\mathrm{Na}^{+}$bound state (Figure 4.25). The 3-4 loop is not well resolved

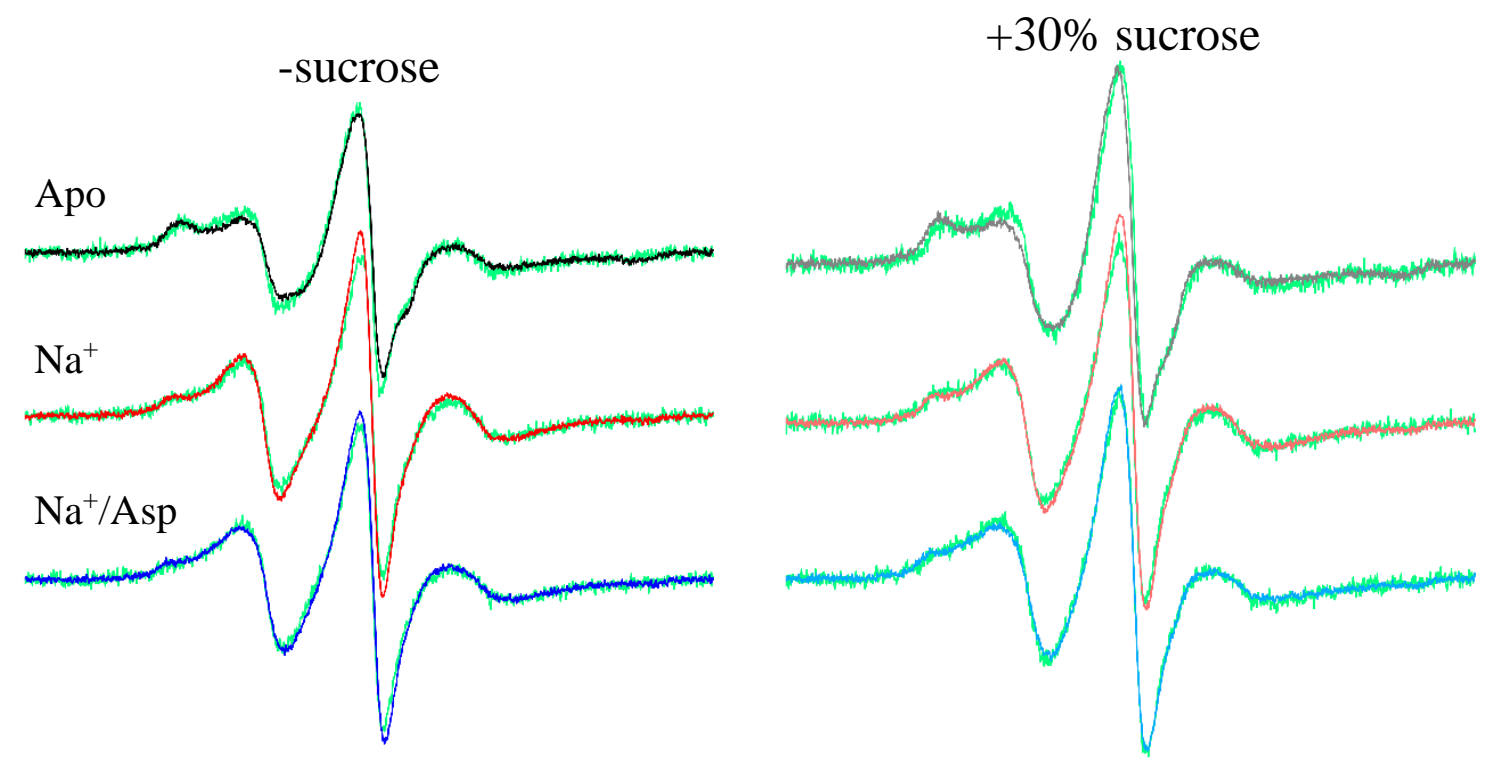

Figure 4.25. CW-EPR spectra for K125R1 with chloride or gluconate. Lineshape changes were only observed for the substrate free state. 
in the crystal structure, making the identification of the two components difficult. However, is it clear that gluconate substitution differentially affects sites that are important for transport. 
Chapter 5

Discussion and Concluding Remarks 
The purpose of this thesis was to determine whether the currently available crystal structures of $\mathrm{Glt}_{\mathrm{ph}}$ capture biologically relevant substates under conditions that support transport. Crystal structures have captured $\mathrm{Glt}_{\mathrm{ph}}$ in multiple conformations. The outward facing conformation showed all monomers facing the same direction (Boudker et al., 2007; Boudker et al., 2007; Verdon et al., 2013). The inward facing conformation, which was captured via crosslinking, showed all monomers facing inward (Reyes et al., 2009; Verdon et al., 2014). The asymmetric conformation captured two monomers in the inward facing conformation and one in an intermediate conformation (Verdon and Boudker, 2012). The intermediate structure, which was determined from the gain-offunction double mutant R276S/M395R, captured a conformation in which the transport domain was unlocked from the trimerization domain (Akyuz et al., 2015). These conformations may be different than biologically relevant conformations, however, due to the precipitants and detergents needed for crystallization and the absence of a lipid bilayer. CW experiments in a lipid bilayer and pulsed EPR experiments in both detergent micelles and a lipid bilayer provided evidence for conformations that have not been captured by the crystal structures.

Previous studies on conformational heterogeneity during transport have provided conflicting results. Stabilization of the inward or outward facing conformations in the presence of $\mathrm{Na}^{+} /$Asp appeared to be site-specific with spin labels at S300 (TM 7) and A364 (HP2) showing distances corresponding to the inward facing conformation and S331, which is on a loop between TM 7 and HP2, showing the protein in the outward facing conformation (Hanelt et al., 2013). A separate study shows distances corresponding to the outward facing conformation at K290 (HP1) and the inward facing 
conformation at L329 (TM 7) with little change in conformational stabilization in lipid bilayers throughout other sites in the transport domain (Figure A.1) (Georgieva et al., 2013). The preferred conformation measured previously at T375 did not agree with the results presented here showing strong stabilization of the inward facing conformation upon $\mathrm{Na}^{+} /$Asp addition (Figure A.1, Figure 4.3) (Georgieva et al., 2013). While there was most likely error in the populations calculated from the distributions due to the threeGaussian fits (Georgieva et al., 2013), the mean distance centered around $40 \AA$ still did not agree with our results. Single molecule FRET results at N378 and I294 showed stabilization of the outward facing conformation in the presence of $\mathrm{Na}^{+} / \mathrm{Asp}$ (Akyuz et al., 2013). This did not agree with the results for I294 from this group using DEER that showed no change with substrate addition (Figure A.1). Interestingly, the attachment of the conformational restricted R1p spin label, which adds a pyridyl ring, shifted conformational selection in the substrate free and $\mathrm{Na}^{+}$bound states, trapping them in the inward facing conformation and demonstrating that conformational selection may be altered by the choice of probe (Figure 4.6). The bilayer placement based on the hydrophobic length of TM1 placed this site in the bulk aqueous solution, so the choice of label would most likely not affect conformational energetics. However, the stabilization of the inward facing conformation at T375 with R1p shows that there are areas of the protein that are unexpectedly in contact with the lipid bilayer. If the energy difference between inward and outward facing conformations is small, as suggested previously (Georgieva et al., 2013; Hanelt et al., 2013), and labels at T375 display increased lipid contact in the inward facing conformation, then the more hydrophobic R1p would be expected to shift the protein equilibrium towards the inward facing conformation to 
maximize hydrophobic contact with the lipid bilayer. If the addition of a pyridyl ring to the spin-label side chain can dramatically alter the protein conformational exchange, large fluorophores might also be expected to modulate conformational exchange if the labels experience differences in lipid contact between inward and outward facing conformations.

Models based on $\mathrm{Glt}_{\mathrm{ph}}$ crystal structures suggest that approximately $15 \AA$ is aqueous exposed on both the extra- and intracellular sides (Yernool et al., 2004). In contrast, the power saturation results described here demonstrate lipid contact for several sites that had been modeled as aqueous exposed such as T375R1, which was shown to interact with the lipid headgroups in the inward facing conformation. According to the crystal structures, this site should always face the aqueous basin, regardless of conformation (Figure 4.2). This suggests that either the transport domain adopts an inward facing conformation that is different than what is captured by the crystal structures or the lipid interface cannot be accurately predicted from the crystal structure. Furthermore, the trimerization domain mutants T166R1 and V176R1 showed substrate dependent membrane accessibility changes, which could be due to movement of nearby TMs, movement of the entire protein in relation to the membrane, or bending and stretching of the membrane to accommodate transport domain movements.

Transport assays also provided evidence for membrane contact. One previous study showed increased transport when several native residues were mutated to cysteine; however, the relative transport decreased greatly after spin labeling (Georgieva et al., 2013). This was especially notable for I294C, T375C, and N378C (Figure A.2), and 
would be expected for sites near the headgroup region as the presence of the spin label would stabilize certain conformations, which could alter the rates of interconversion.

Depth measurements for sites along the 3-4 loop showed increasing membrane exposure along the C-terminal end of the loop. Q121R1 was aqueous exposed, while K125R1 and P128R1 were positioned below the phosphate (Figure 4.10). This increasing membrane exposure would explain previous results that showed decreased fluorescin-5-malemide (FM) labeling starting at residue P124 in the substrate free state (Compton et al., 2010). The depth measurements were taken in the presence of $\mathrm{Na}^{+} / \mathrm{Asp}$ or DL-TBOA, which both increased FM labeling in the previous study (Compton et al., 2010). The CW spectra at K125R1 showed a larger population of the immobile component in the substrate free state. The spin label may be coming into tertiary contact with the lipid bilayer, which would decrease accessibility to FM labeling until substrate binds (Figure A.3). Q121R1 was also inaccessible to labeling with FM until substrate or inhibitor was bound. Although depth measurements were not done in the substrate free state, differences were seen in the CW spectra for Q121R1 between substrate free and substrate bound states, indicating that interface exposure at any point during transport is unlikely.

HP1, which has been implicated as the intracellular gate, also appeared to undergo transitions that have not been captured by the crystal structures. HP1 is shown in every crystal structure in a closed conformation that is tightly coupled to TM 8. The environment around HP1 is crowded in the outward facing conformation, with TMs 2 and 7 within 6-10 $\AA$ of the loop's apex. The environment is less restrictive in the inward 
facing conformation as HP1 only interacts with TM 8. The CW spectra at S277R1 and S279R1 showed two to three populations with the most mobile component representing approximately $1 \%$ of the total (Figure $4.10 \mathrm{~b}$ ). This component could be an open state of HP1 that is not tightly coupled to TM 8 and can release substrate. Since the population of this open conformation is small, it would not be captured crystallographically. This agrees with simulations that have shown increased dynamics for HP1 during and directly following substrate release (Zomot and Bahar, 2013).

Previous research has shown that the identity of the lipid headgroups altered transport rates (McIlwain et al., 2015). POPE had the highest transport rate, and POPC had the lowest rate. Therefore, it is possible that the different lipid systems used for $\mathrm{Glt}_{\mathrm{ph}}$ reconstitution could partly explain conflicting results. The CW spectra for V176R1 were similar when POPC was compared to POPE, which would be expected for a site in the trimerization domain that was not interacting with the lipid headgroups (Figure 4.14). However, the CW spectra for T166R1 were more mobile in POPC than POPE in a substrate dependent manner (Figure 4.15). It is possible that conformational changes that occur when $\mathrm{Na}^{+}$binds allow the spin label to interact more strongly with the lipid headgroups.

The DEER distributions for T375R1 showed changes in the substrate free and $\mathrm{Na}^{+}$ bound states but not when $\mathrm{Na}^{+} /$Asp was bound in when POPC was substituted for POPE. This suggests that there is conformational flexibility in the inward facing conformation that alters the local environment around the label but doesn't change the distance between labels. The substrate dependence of the lipid effect also shows how movements between the outward and inward facing conformations are different in substrate free and substrate 
bound states. This conflicts with the crystal structures of the substrate-free and substrate bound protein that suggest the only structural difference in the transport domain is the formation of the substrate binding site.

Mutagenesis studies have identified residues that comprise the chloride ion channel, but the conductance has not been linked to conformational exchange events during transport (Ryan et al., 2004; Ryan and Mindell, 2007). Gluconate substitution broadened the $\mathrm{Na}^{+} /$Asp bound spectrum when $30 \%$ sucrose was added (Figure 4.24) and increased spin label mobility when the transport inhibitor DL-TBOA was bound (Figure 4.24). This shows substrate dependent conformational changes when no chloride is present, suggesting that the channel is not forming properly without chloride. Mobility also increased at K125R1 for the substrate free state (Figure 4.25). The mobility increase for K125R1 was not expected for substrate-gated chloride conductance at sites that are not in the chloride ion channel, which should not experience changes when substrate is not bound. While these experiments do not rule out specific chloride effects on conformational exchange, it is clear that sites in the transport domain are differentially affected by chloride removal.

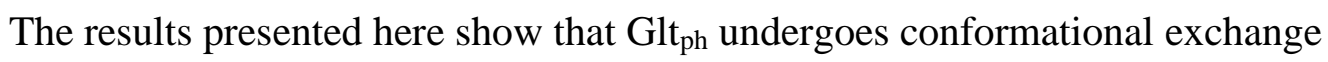
events that have not been captured crystallographically, most likely due to osmolyte and detergent effects. Furthermore, conformational exchange is altered by lipid bilayer properties, the presence or absence of chloride, and the type of site specific probe that is used. Future research should continue to define contributions of both the lipid bilayer and the chloride ion conductance on conformational exchange events. Power saturation at 
sites suspected to be in contact with the center of the bilayer and at the membrane aqueous interface would determine membrane thickness. Crosslinking the protein in either the outward or inward facing conformations for power saturation experiments would map the membrane interface in conformations that are responsible for substrate binding and release and would remove the spectral averaging due to the exchange.

The effect of different spin labels on sampled conformations could be investigated by placing R1 and R1p at several transport and trimerization domain sites for DEER. It is possible that the label primarily effects interface sites by interacting differently with the membrane. Finding sites in which the choice of label does not affect the distance distributions would provide a more accurate picture of conformational exchange events. To see if the label alters conformational exchange in unlabeled helices, a phenylalanine could be substituted at sites such as T375 while other TMs are labeled for DEER. Furthermore, larger fluorophores could be attached at sites that are always solvent exposed, and the FRET efficiencies could be compared to sites that have expected lipid contact. The distances calculated from the FRET efficiencies could then be compared to DEER distance distributions at these sites.

Glt $_{\text {ph }}$ crystals are grown using the protective osmolyte PEG 400. However, PEG 400 aggregated protein loaded vesicles and was unsuitable for EPR experiments. Detergent solubilized and reconstituted $\mathrm{Glt}_{\mathrm{ph}}$ could be attached to a solid support via the his-tag to compare the effects of the crystallography buffer and transport buffer on sampled conformations in substrate free and substrate loaded states.

Since the entire chloride ion channel has not been experimentally determined, crystallizing Glt $\mathrm{ph}_{\mathrm{p}}$ with gluconate substituted for chloride would provide a starting point 
for determining the ion channel and its effects on conformational exchange by potentially showing changes in the structure around sites that have been shown to be part of the channel. This would guide the choice of site for DEER experiments to compare sampled conformations in the presence and absence of chloride. 
Chapter 6

Appendix 
a Intracellular
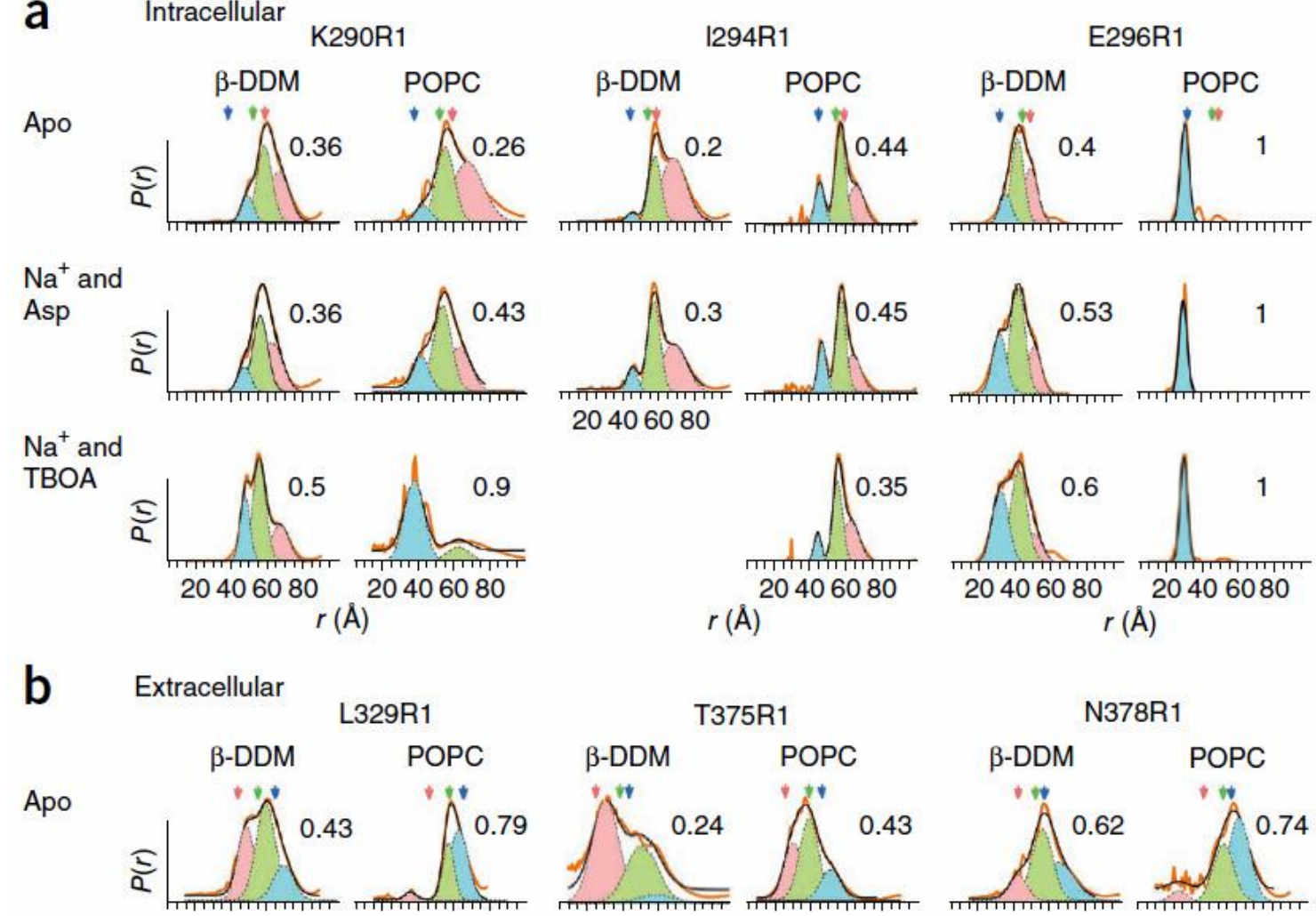

$\mathrm{Na}^{+}$and

Asp
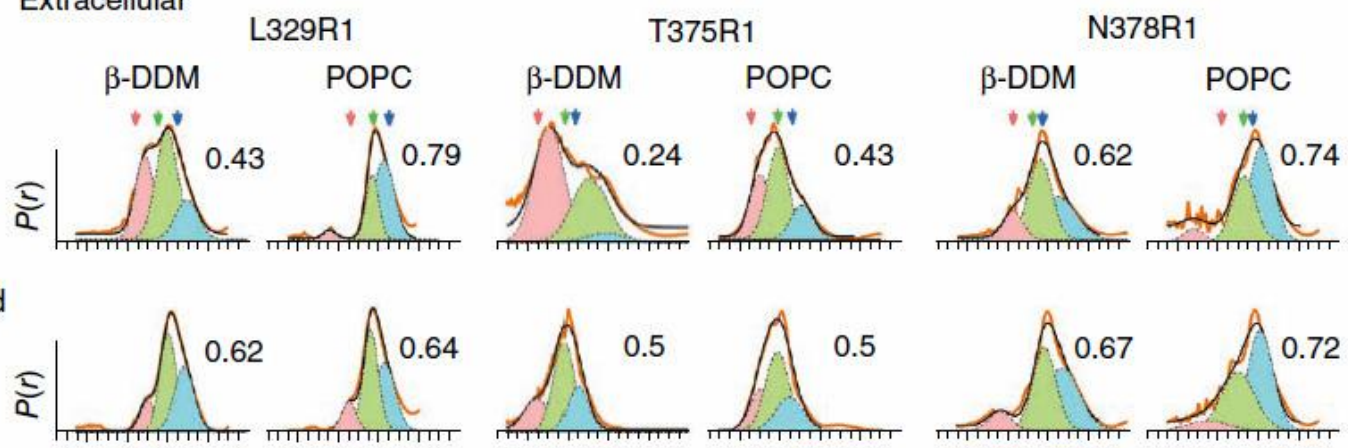

$\mathrm{Na}^{+}$and

TBOA
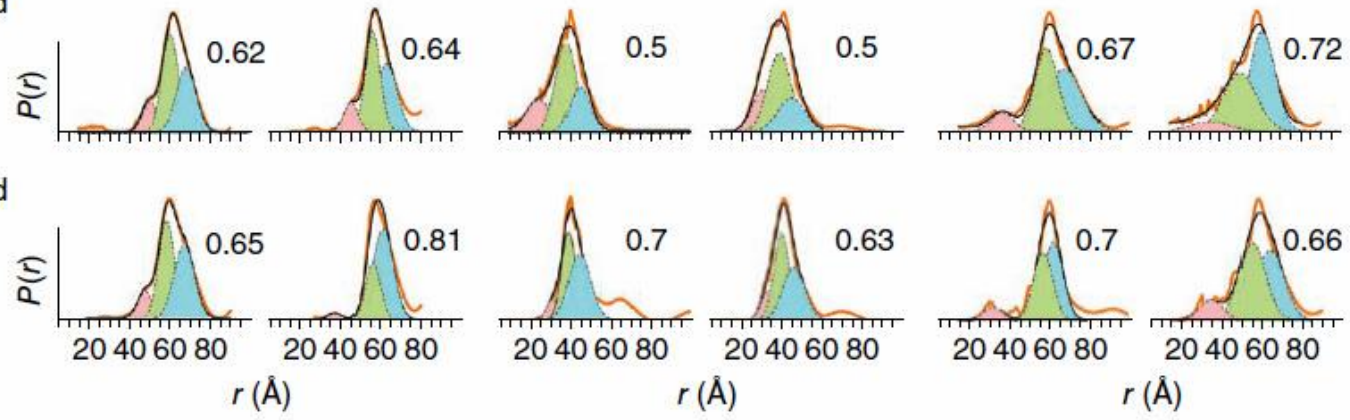

Figure A.1. DEER distance distributions for select transport domain sites. The outward facing, asymmetric, and inward facing conformations are represented by the blue, green, and pink Gaussians, respectively. Assuming the right side out orientation during reconstitution, a) intracellular facing sites. K290 is on HP1, I294 and E296 are on the loop between HP1 and TM 7. b) L329 is on TM 7, and T375 and N378 are on the loop between HP2 and TM 8. (Adapted from Georgieva et al., 2013). 


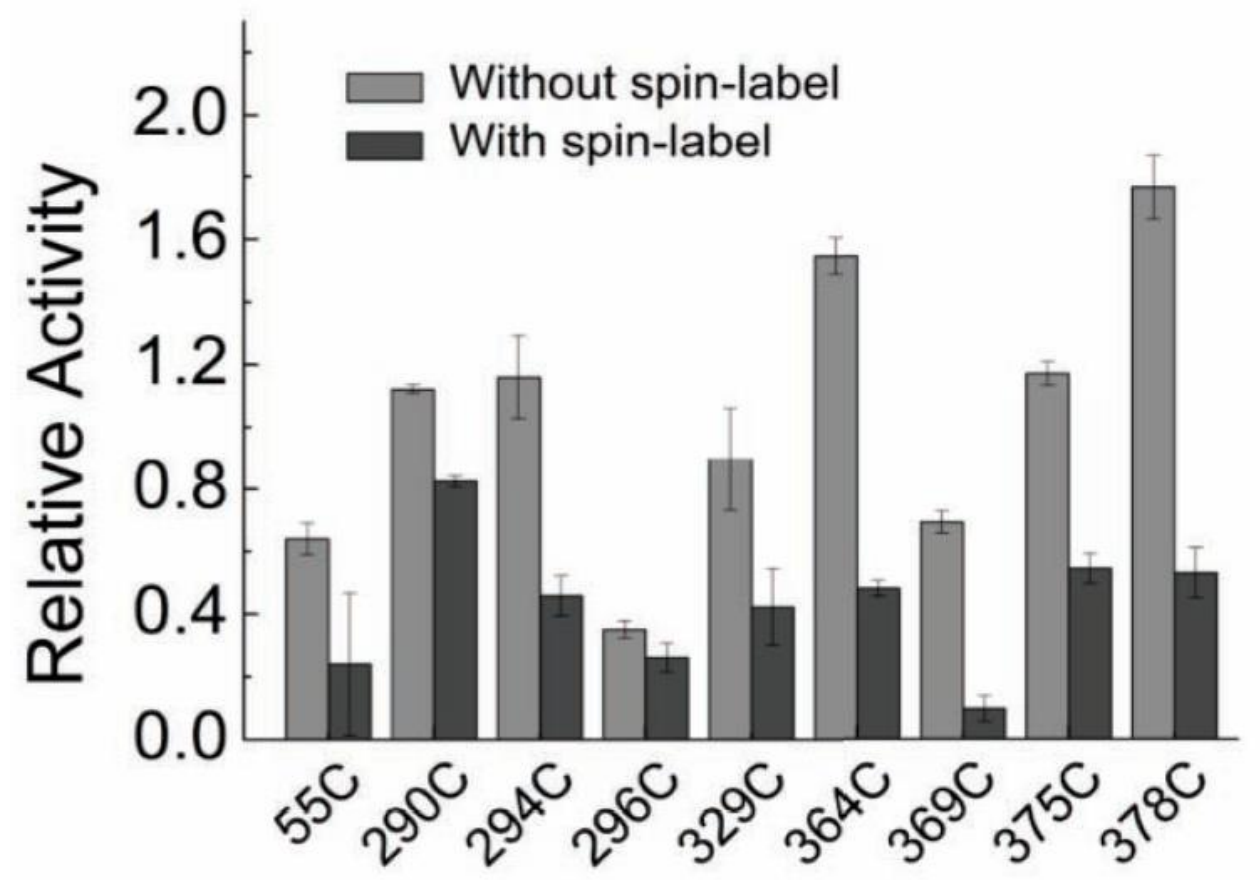

Figure A.2. Aspartate uptake of select transport domain sites compared to wild type of control before and after spin labeling. Wild type cys-less $\mathrm{Glt}_{\mathrm{ph}}$ and single cysteine mutants before were reconstituted into POPC liposomes for transport assays. Background uptake was determined in the absence of $\mathrm{Na}^{+}$gradient and subtracted. (Adapted from Georgieva et al., 2013).

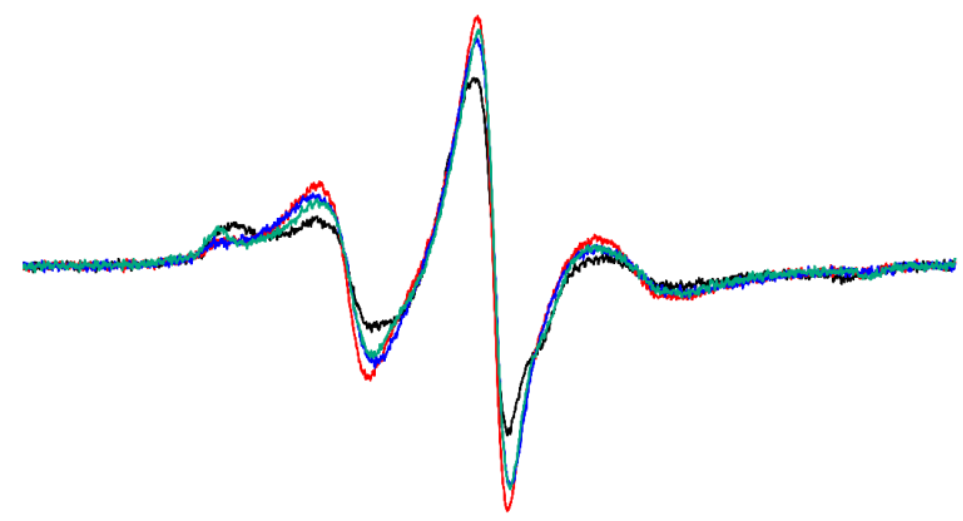

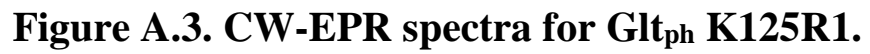

There was a mobility increase upon $\mathrm{Na}^{+}$binding that was slightly modulated by Asp or DL-TBOA binding. 

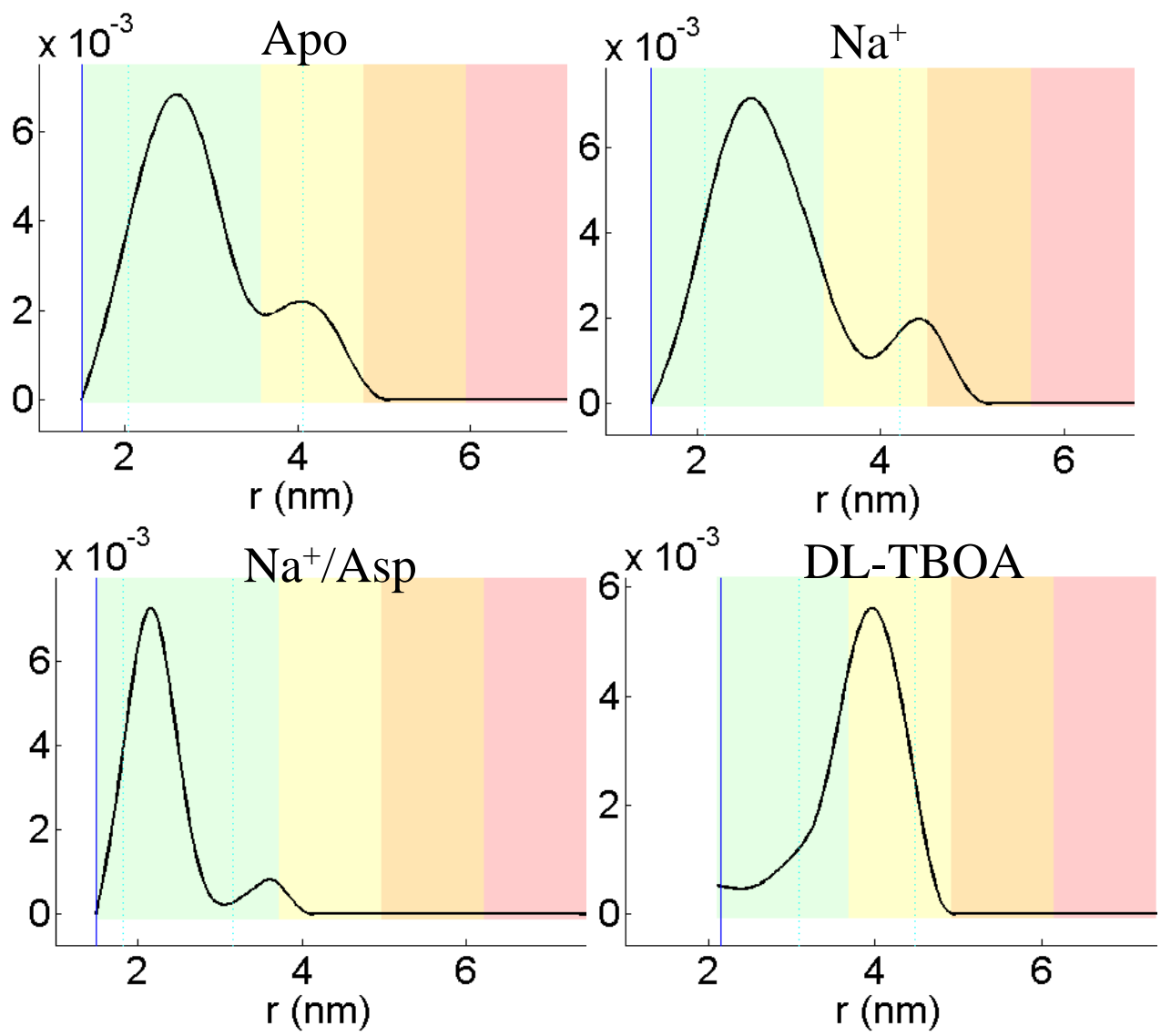

Figure A.4. DEER distance distribution reliability for T375R1 in 3:1 E.coli polar lipids:POPE liposomes.

Green: reliable mean distance, width, and shape

Yellow: reliable mean distance and width

Orange: reliable mean distance

Red: recognition of long distance but no other reliable information These reliability plots correspond to Figure 4.3. 

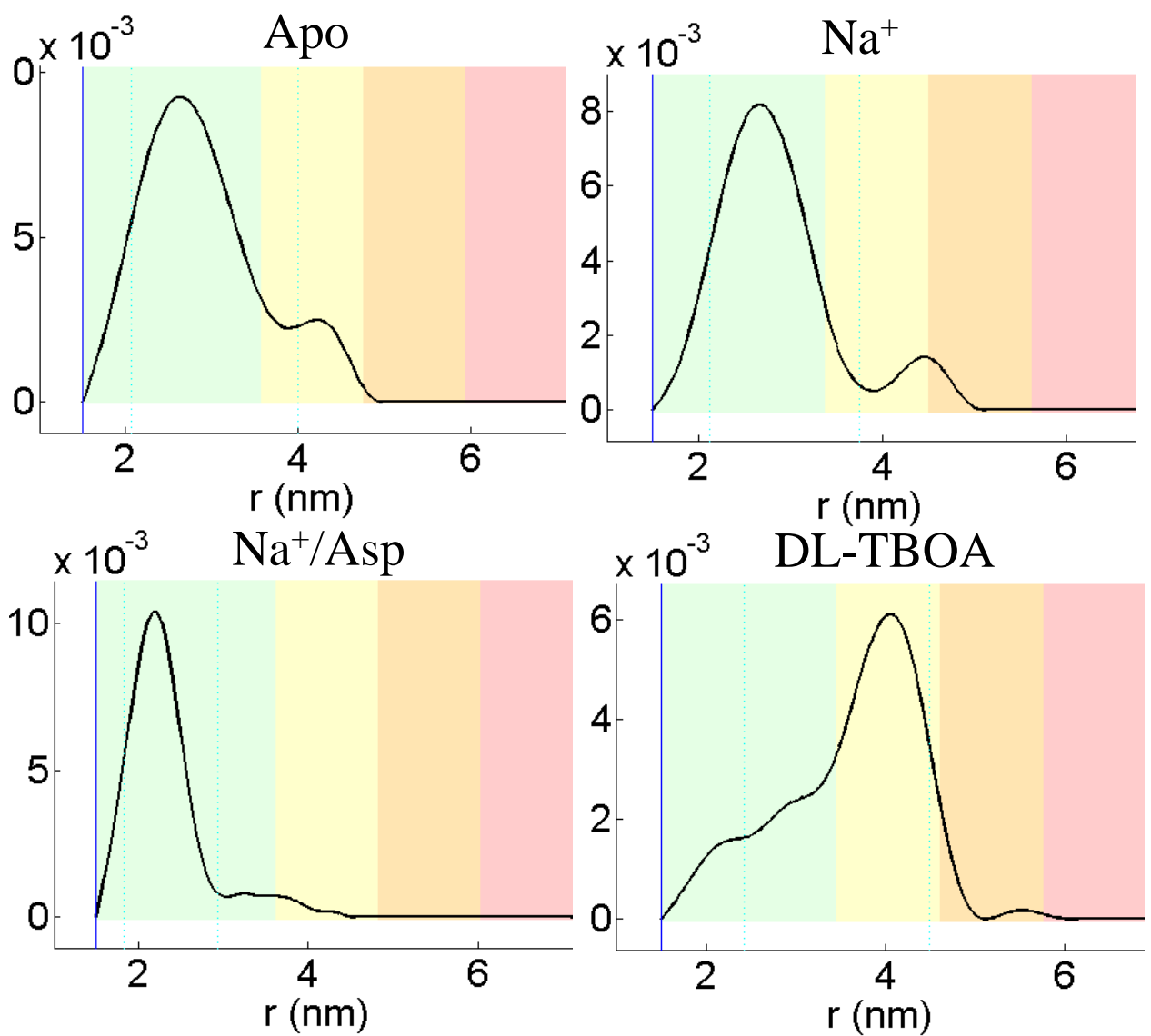

Figure A.5. DEER distance distribution reliability for T375R1 in 3:1 E.coli polar lipids:POPE liposomes with $30 \%$ sucrose.

Green: reliable mean distance, width, and shape

Yellow: reliable mean distance and width

Orange: reliable mean distance

Red: recognition of long distance but no other reliable information

These reliability plots correspond to Figure 4.3. 

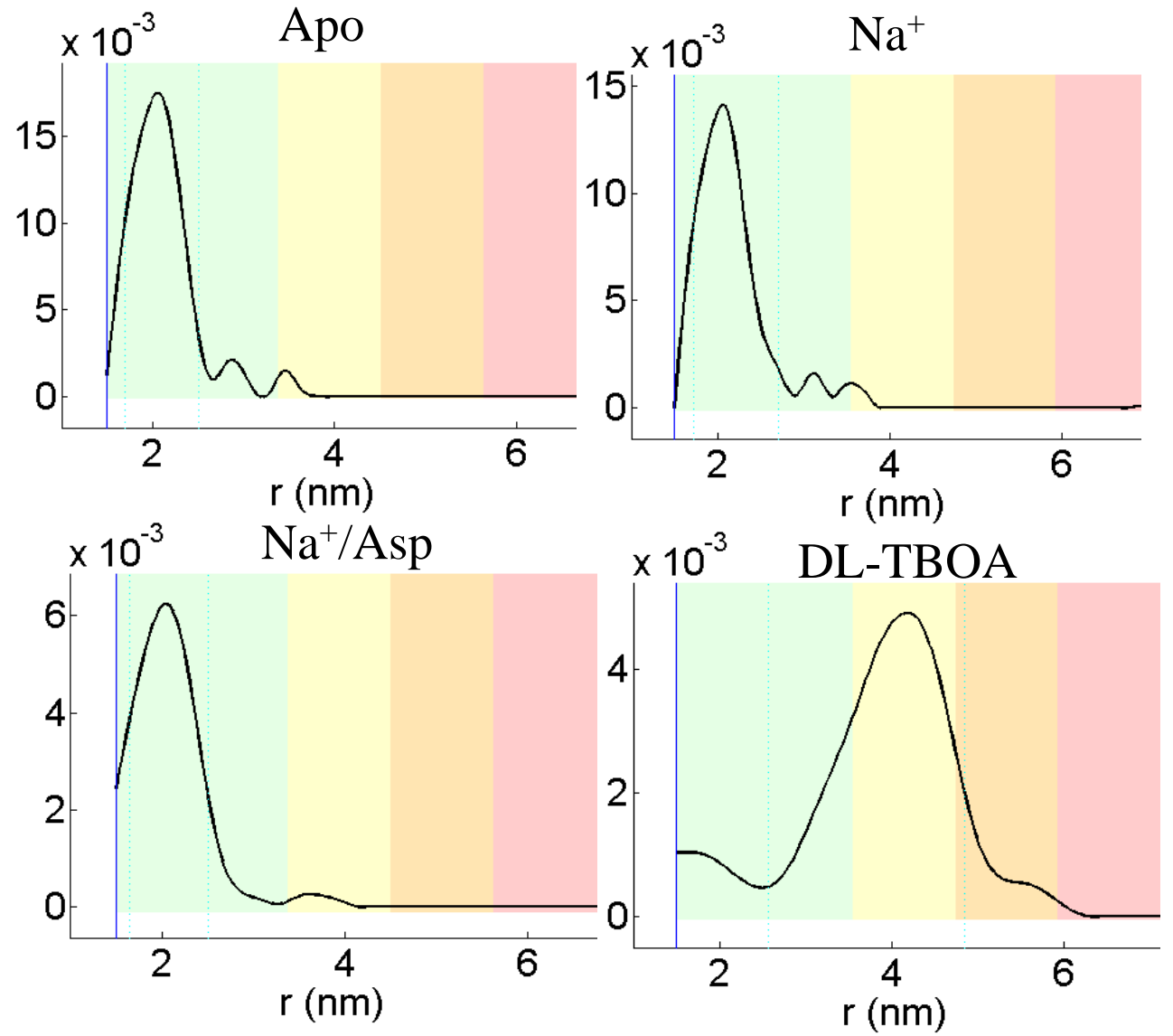

Figure A.6. DEER distance distribution reliability for T375R1p in 3:1 E.coli polar lipids:POPE liposomes.

Green: reliable mean distance, width, and shape Yellow: reliable mean distance and width

Orange: reliable mean distance

Red: recognition of long distance but no other reliable information These reliability plots correspond to Figure 4.5. 

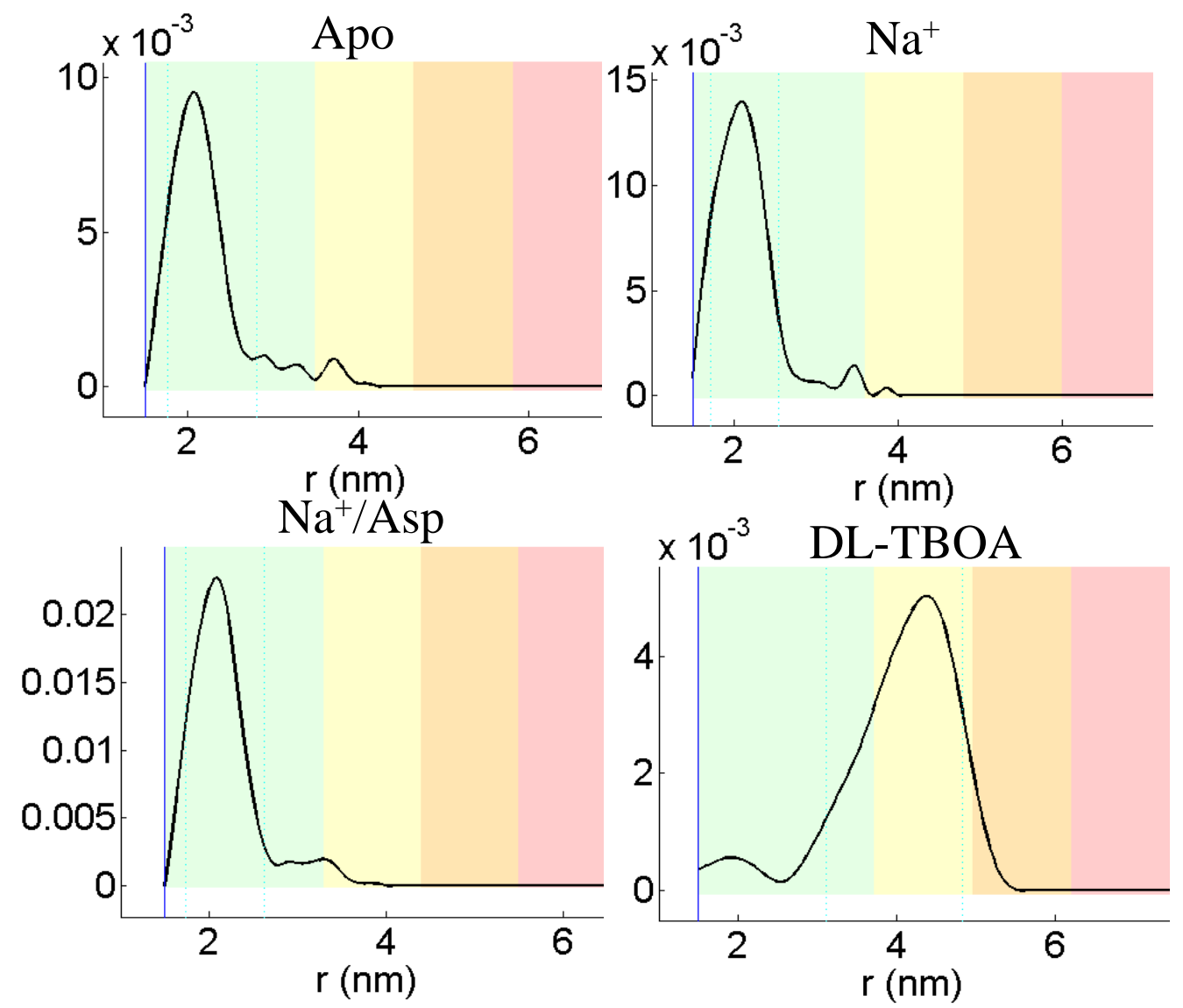

Figure A.7. DEER distance distribution reliability for T375R1p in 3:1 E.coli polar lipids:POPE liposomes with $30 \%$ sucrose.

Green: reliable mean distance, width, and shape

Yellow: reliable mean distance and width

Orange: reliable mean distance

Red: recognition of long distance but no other reliable information These reliability plots correspond to Figure 4.5. 

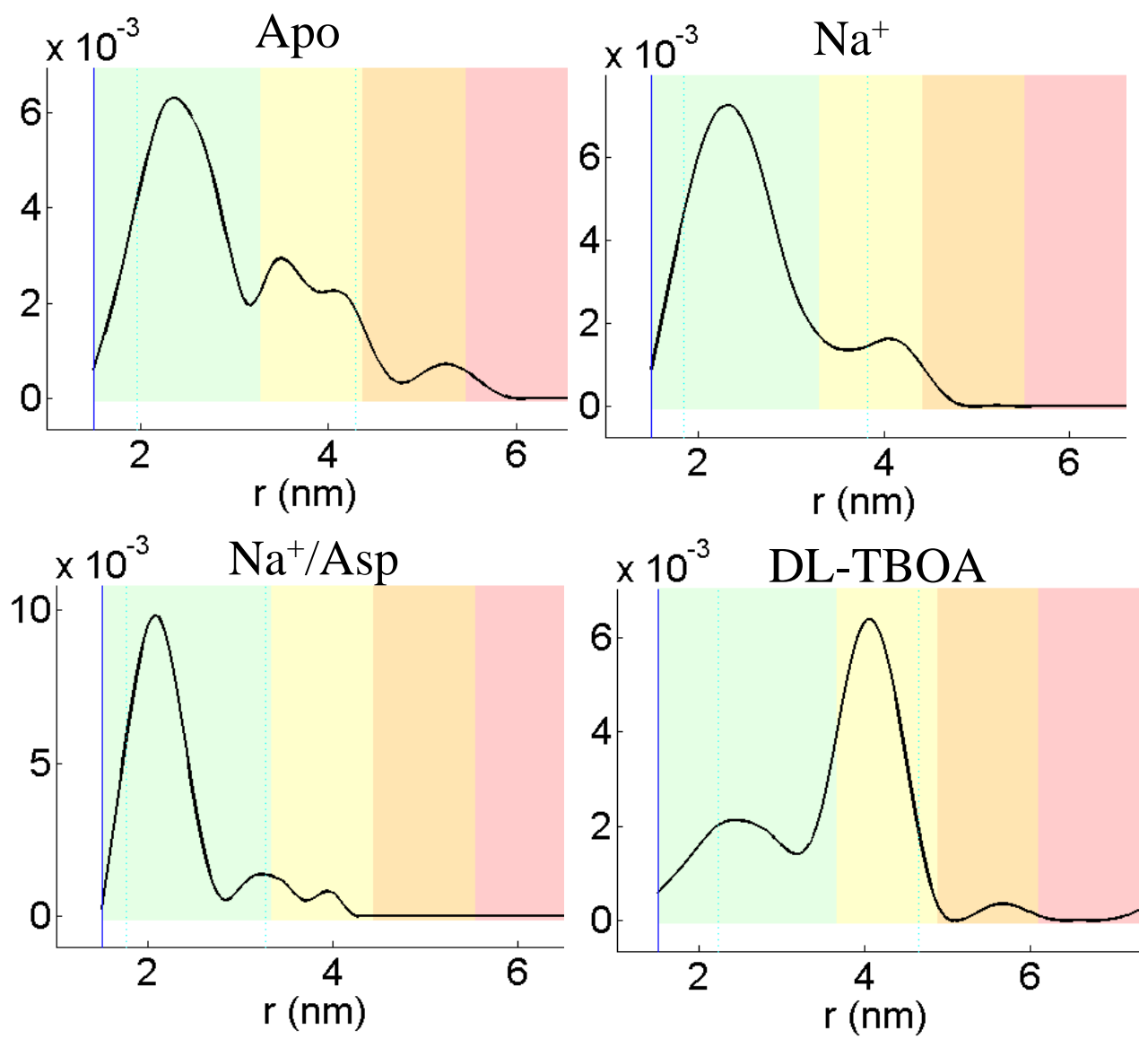

Figure A.8. DEER distance distribution reliability for T375R1 in 3:1 E.coli polar lipids:POPC liposomes.

Green: reliable mean distance, width, and shape Yellow: reliable mean distance and width Orange: reliable mean distance

Red: recognition of long distance but no other reliable information These reliability plots correspond to Figure 4.23 (purple). 

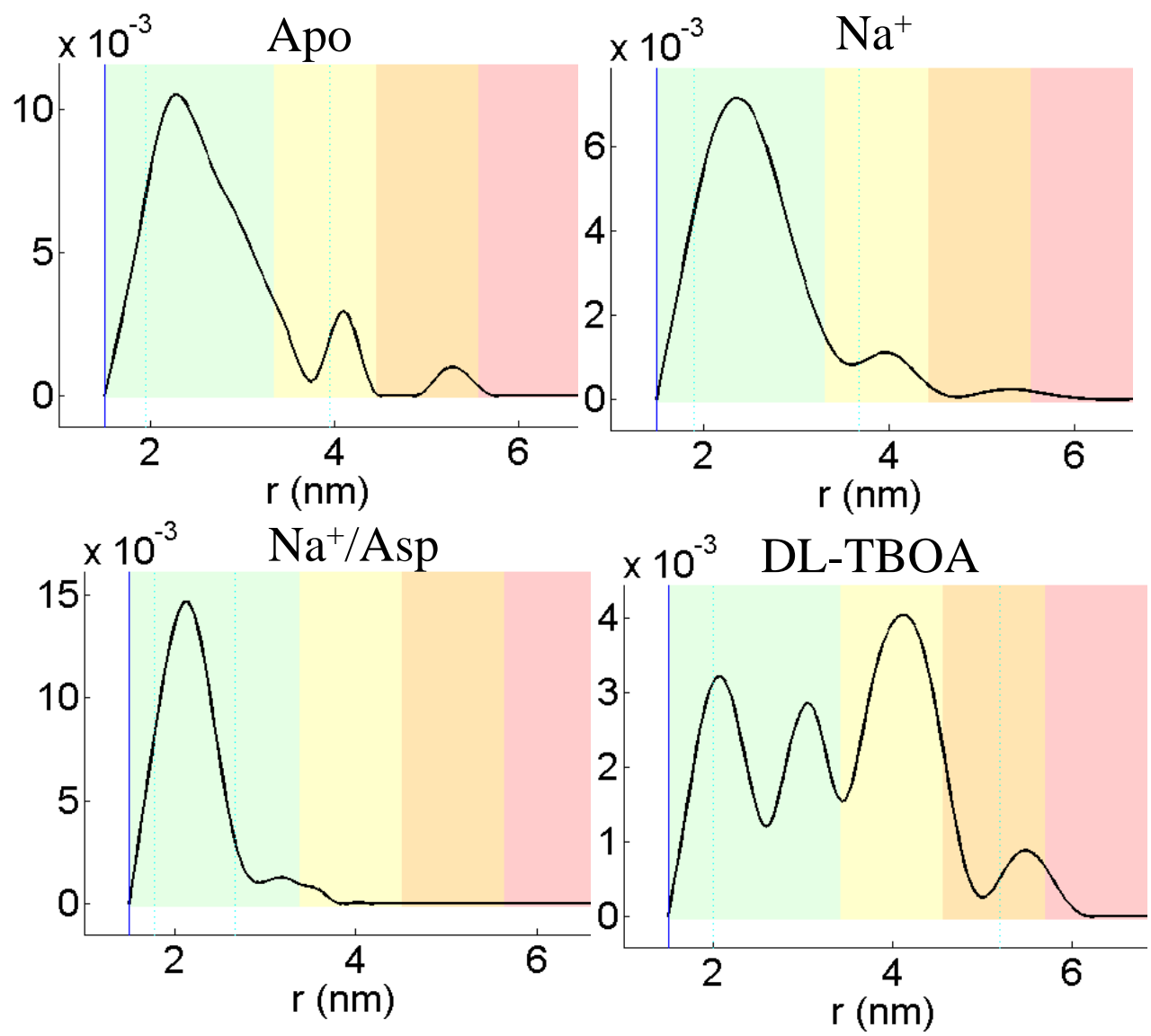

Figure A.9. DEER distance distribution reliability for T375R1 in 3:1 E.coli polar lipids:POPC liposomes with $30 \%$ sucrose.

Green: reliable mean distance, width, and shape

Yellow: reliable mean distance and width Orange: reliable mean distance

Red: recognition of long distance but no other reliable information These reliability plots correspond to Figure 4.24 (purple). 

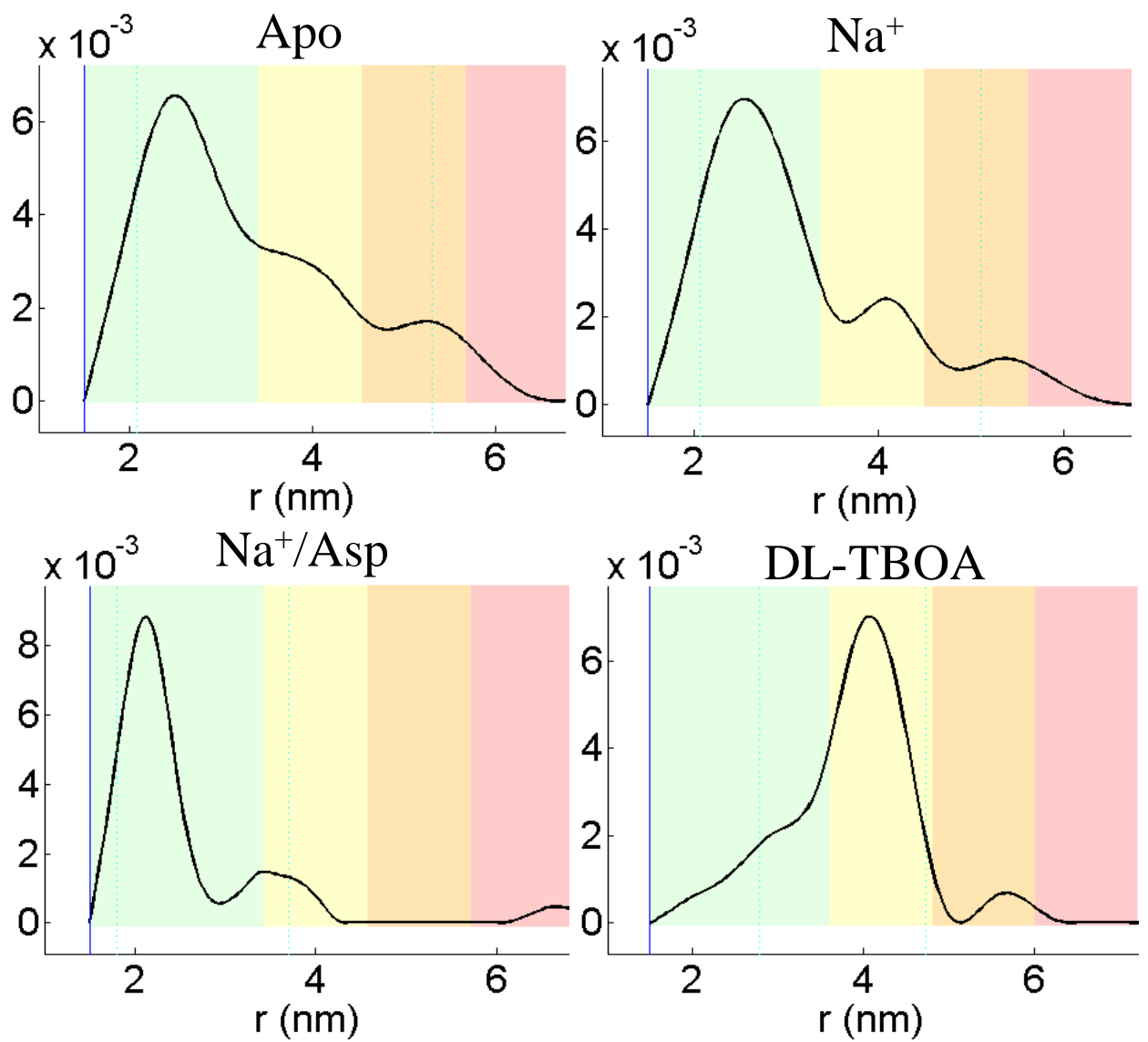

Figure A.10. DEER distance distribution reliability for T375R1 in 3:1:1 DOPE:DOPC:DOPG liposomes.

Green: reliable mean distance, width, and shape Yellow: reliable mean distance and width Orange: reliable mean distance Red: recognition of long distance but no other reliable information These reliability plots correspond to Figure 4.23 (light teal). 

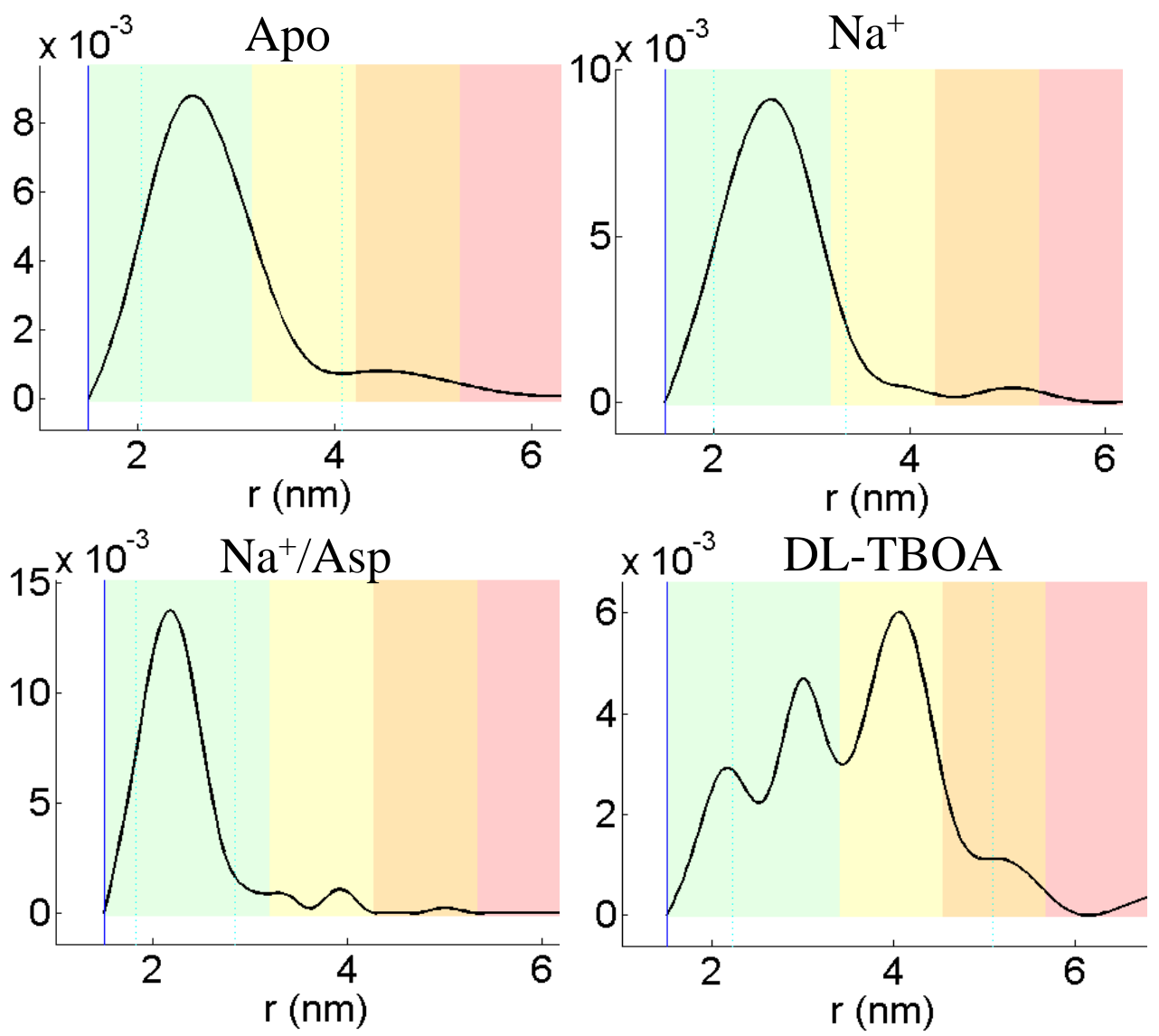

Figure A.11. DEER distance distribution reliability for T375R1 in 3:1:1 DOPE:DOPC:DOPG liposomes with $30 \%$ sucrose.

Green: reliable mean distance, width, and shape

Yellow: reliable mean distance and width

Orange: reliable mean distance

Red: recognition of long distance but no other reliable information

These reliability plots correspond to Figure 4.24 (light teal). 

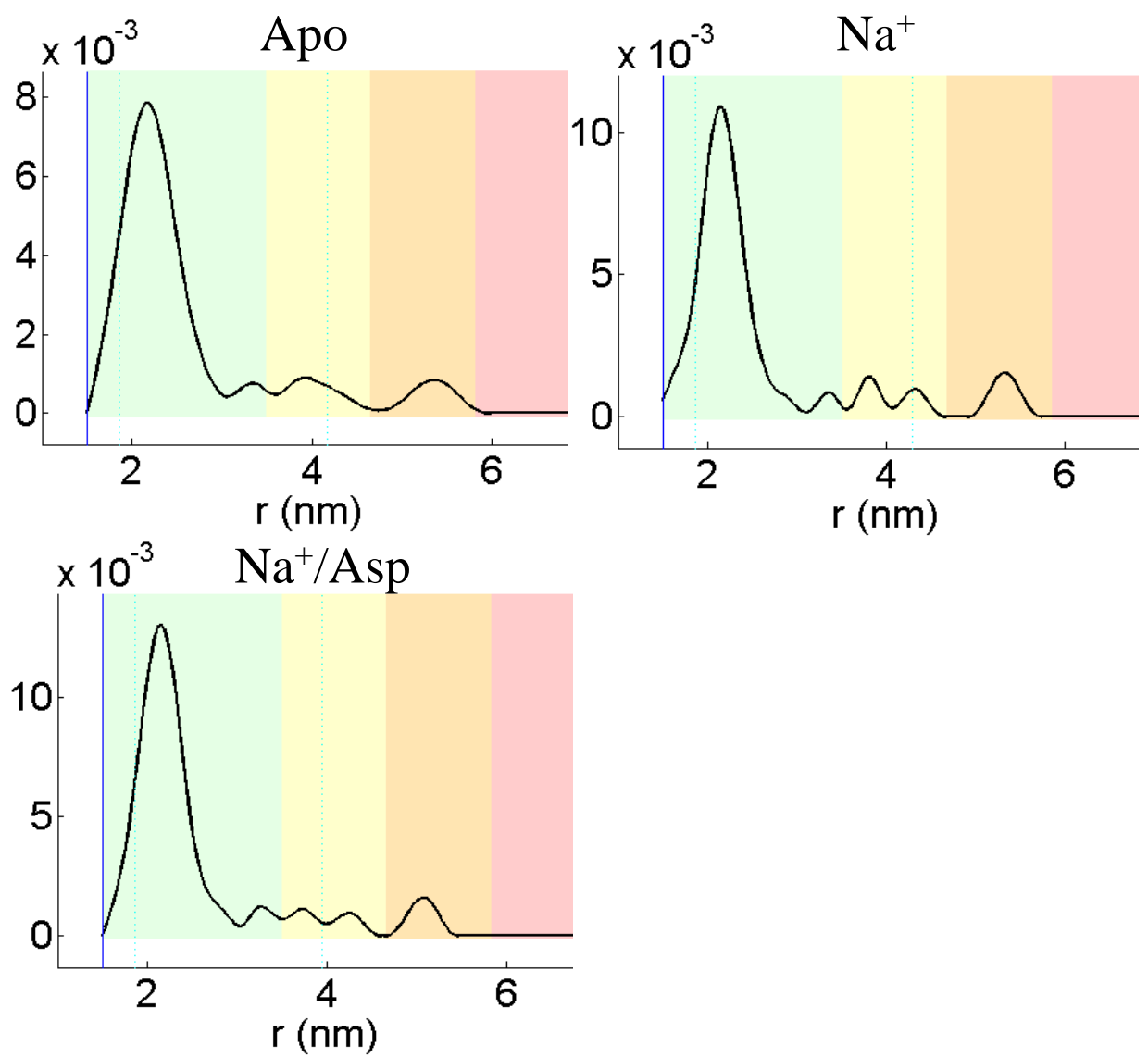

Figure A.12. DEER distance distribution reliability for T375R1 in DDM detergent micelles.

Green: reliable mean distance, width, and shape

Yellow: reliable mean distance and width

Orange: reliable mean distance

Red: recognition of long distance but no other reliable information These reliability plots correspond to Figure 4.6. 

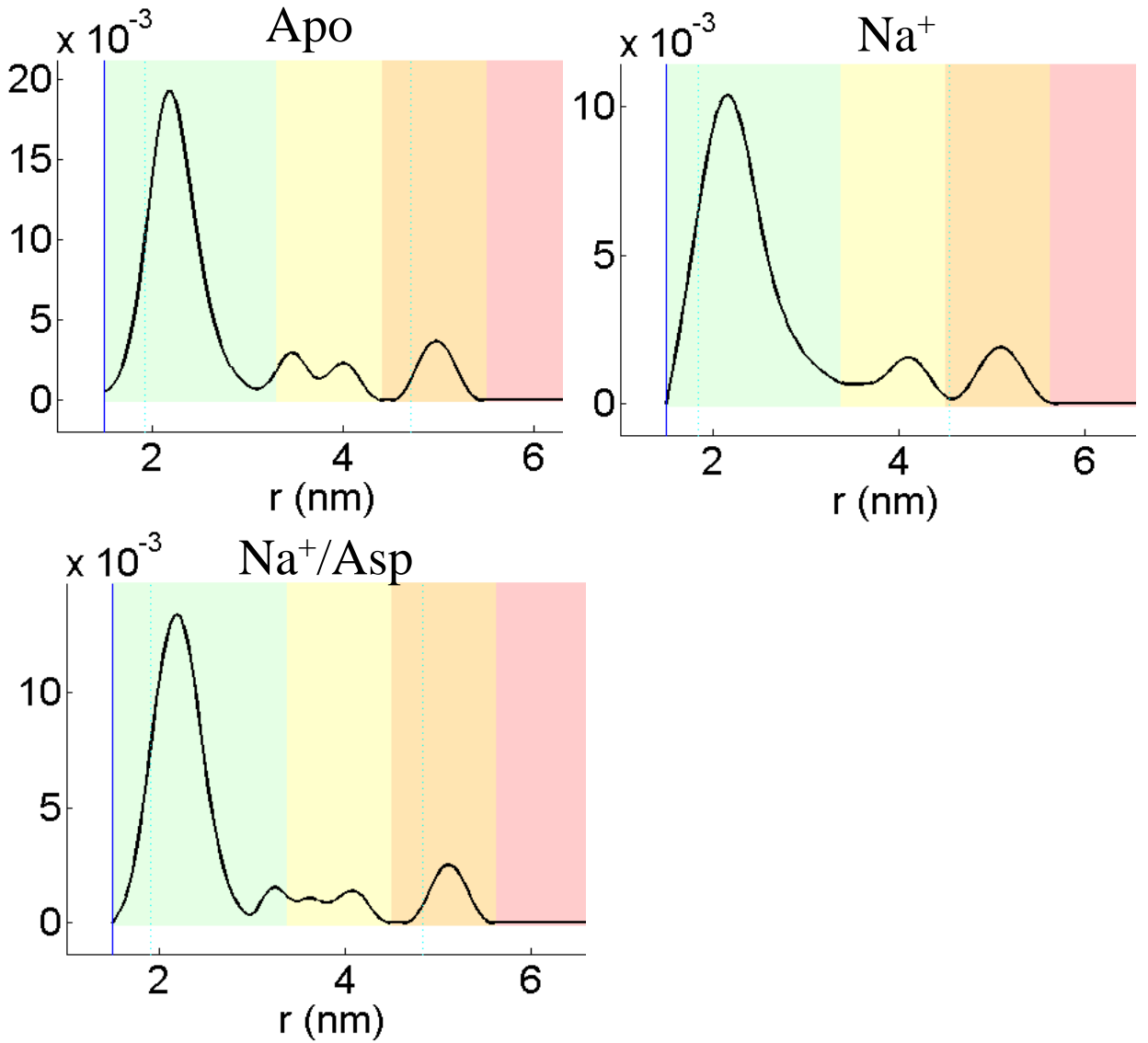

Figure A.13. DEER distance distribution reliability for T375R1 in DDM detergent micelles with $\mathbf{3 0 \%}$ sucrose.

Green: reliable mean distance, width, and shape

Yellow: reliable mean distance and width

Orange: reliable mean distance

Red: recognition of long distance but no other reliable information These reliability plots correspond to Figure 4.6. 


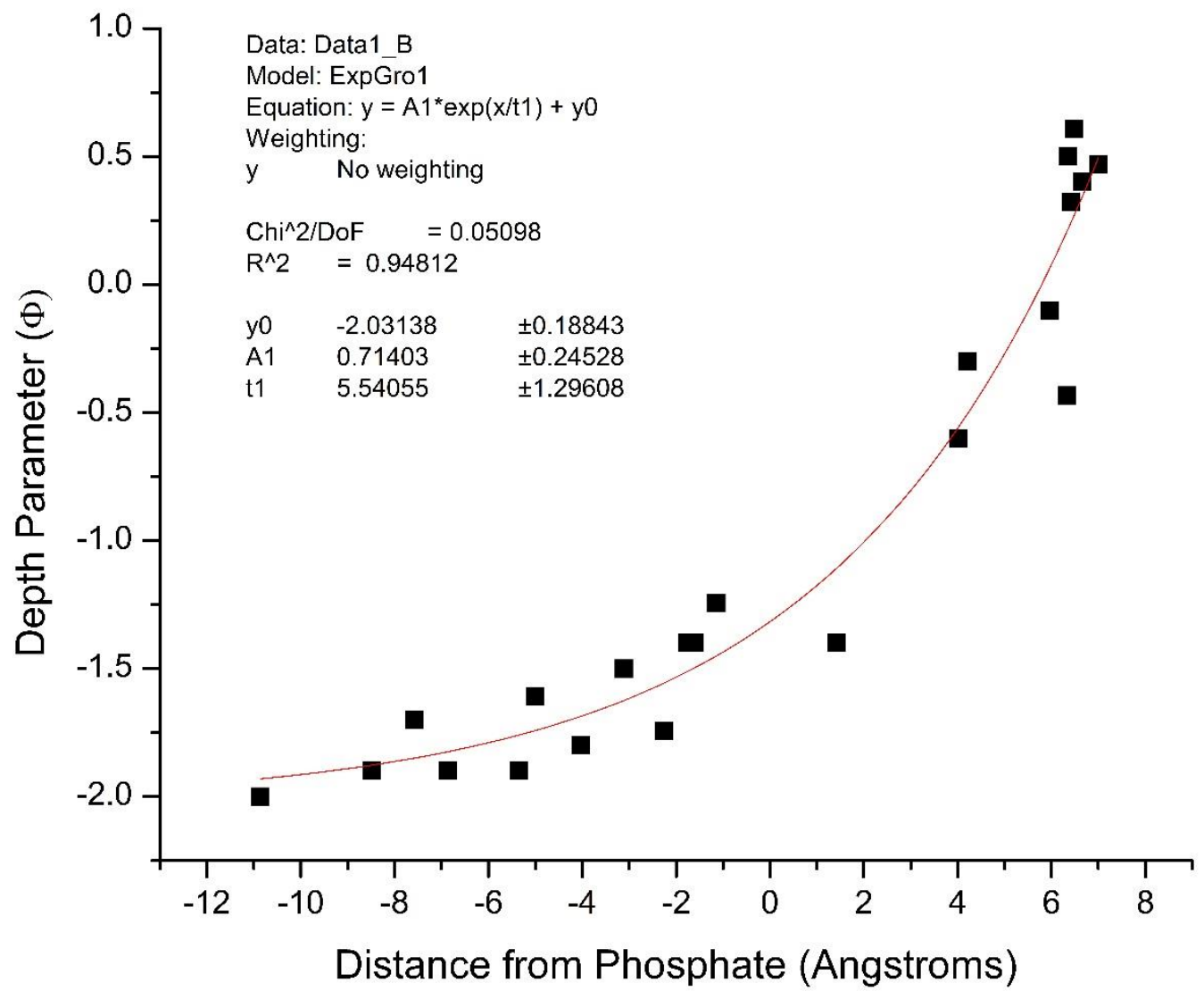

Figure A.14. Calibration curve used for the Power Saturation experiments. 


\section{Chapter 7}

Materials and Methods 


\subsection{Protein Mutagenesis, Expression, and Purification}

Plasmids for Gltph cysteineless wild type, Q121C, K125C, P128C, T166C, V176C, V274C, T275C, S277C, and S279C were kindly provided by Dr. Joseph Mindell. The mutation T375C was introduced into the provided cysteineless pBAD plasmid with mutagenic primers (Integrated DNA Technologies, Coralville, IA) through site directed mutagenesis using Pfu turbo polymerase (Agilent Technologies, Santa Clara, CA). Plasmids were amplified in TOP10 chemically competent cells and purified using a Qiagen Miniprep Column (Qiagen, Hilden, Germany); sequences were confirmed by DNA sequencing (GeneWiz, South Plainfield, NJ).

Glt $\mathrm{ph}_{\mathrm{w}}$ with an octahistidine purification tag was grown and expressed in TOP10 cells. Cells were grown in Luria Broth (LB) with an ampicillin (Gold Biotechnology, Olivette, MO) concentration of $100 \mu \mathrm{g} / \mathrm{mL}$ at $37^{\circ} \mathrm{C}$ until the O.D.600 was $0.8-1.0$. For protein expression arabinose (Alfa Aesar, Haverhill, MA) was added at a concentration of $0.1 \%$, and cells were harvested 4-6 hours after induction. The inner membrane was isolated through physical lysis using a French Press followed by ultrcentrifugation at 41,000 rpm (Bekman-Coulter, Brea, CA). The protein was solubilized in 1x NiNTA buffer (20 mM Tris-amino, pH 7.4, $200 \mathrm{mM} \mathrm{KCl)} \mathrm{and} 2 \mathrm{mM} \mathrm{n}$-dodecyl- $\beta$-D-maltoside (DDM, Anatrace, Maumee, $\mathrm{OH}$ ) on ice for 1 hour followed by ultracentrifugation at 41,000 rpm. Purification buffers containing $20 \mathrm{mM}$ Tris-amino, pH 7.4, $200 \mathrm{mM} \mathrm{KCl,}$ and $2 \mathrm{mM}$ DDM were used throughout the purification process; the wash buffer also contained $60 \mathrm{mM}$ imidazole (Fisher Scientific, Waltham, MA), and the elution buffers contained either $250 \mathrm{mM}$ or $500 \mathrm{mM}$ imidazole. The protein was loaded onto NiNTAagarose resin (Qiagen, Hilden, Germany) and incubated at $4^{\circ} \mathrm{C}$ for 1 hour. Following 
elution, protein concentration was quantified on the NanoDrop using the molar extinction coefficient. Purified protein was incubated with thrombin and (1-Oxyl-2,2,5,5tetramethylpyrroline-3-methyl)methanethiosulfonate (MTSL) at room temperature overnight. The buffer was exchanged to storage buffer (20 mM Tris-amino, $\mathrm{pH}$ 7.4, 200 mM KCl, 2 mM DDM) using a PD10 size exclusion column (GE Healthcare Lifesciences, Pittsburgh, PA) for use in reconstitution and storage at $-80^{\circ} \mathrm{C}$.

\subsection{Protein Reconstitution}

For reconstitution in liposomes, a 3:1 (w:w) mixture of E.coli polar lipid extract and either POPC or POPE or a 3:1:1 mixture of DOPE:DOPC:DOPG (Avanti polar lipids, Alabaster,AL) was dried under nitrogen for 2 hours followed by a 15 minute incubation in $\mathrm{D}_{2} \mathrm{O}$ Apo Buffer (40 mM HEPES, $\mathrm{pH}$ 7.4, $200 \mathrm{mM}$ KCL, diluted in $\mathrm{D}_{2} \mathrm{O}$ ). The liposomes were homogenized after 10 freeze-thaw cycles by extruding 11 times through a $400 \mathrm{~nm}$ pore size polycarbonate filter (GE Healthcare Lifesciences, Pittsburgh, PA). Liposomes were subsequently diluted in Apo Buffer (40 mM HEPES, pH 7.4, $200 \mathrm{mM} \mathrm{KCL}$, diluted in $\mathrm{H}_{2} \mathrm{O}$ ) to a final concentration of $4 \mathrm{mg} / \mathrm{mL}$ and were destabilized by stepwise addition of Triton X100 (10\% w/v) until the O.D.540 reached 2/3 maximum absorbance. Purified protein was added in a 1:50 protein:lipid ratio (w/w). The proteoliposomes were incubated at room temperature for 15 minutes. Biobeads SM-2 (Bio-Rad, Hercules, CA) $(25 \mathrm{mg} / \mathrm{mL})$ were added, and the mixture was incubated on a rocker at room temperature for another 30 minutes. After another Biobead addition, the mixture was incubated at $4{ }^{\circ} \mathrm{C}$ for 1 hour, followed by another Biobead addition and incubation at $4{ }^{\circ} \mathrm{C}$ overnight. After the overnight incubation, another Biobead addition 
was incubated at $4^{\circ} \mathrm{C}$ for 2 hours. The Biobeads were removed, and ultracentrifugation at 80,000 rpm pelleted the liposomes. Pellets were resuspended in Apo Buffer to a final lipid concentration of $100 \mathrm{mg} / \mathrm{mL}$ for storage at $-80^{\circ} \mathrm{C}$.

\subsection{Power saturation EPR}

Power saturation EPR experiments were conducted at room temperature on an EMX Xband EPR Spectrometer (Bruker). The microwave power was varied from 0.25 to 100 $\mathrm{mW}$ and 10-16 scans of the central peak were averaged for each power step. The applied magnetic field was swept through $10 \mathrm{G}$ with a modulation amplitude of $1 \mathrm{G}$ and a frequency of $100 \mathrm{kHz}$. The power saturation was conducted on spin-labeled Gltph in the presence of air $\left(20 \% \mathrm{O}_{2}\right), \mathrm{N}_{2}$, or $\mathrm{N}_{2}$ with $10 \mathrm{mM}$ NiEDDA (Victor and Cafiso, 2001). In each of these conditions and for every power step, the amplitude of the central peak was measured and the $\mathrm{P}_{1 / 2}$ value was extracted using a LabVIEW program provided by Christian Altenbach (University of California, Los Angeles). The values of $\Delta \mathrm{P}_{1 / 2}$ for $\mathrm{O}_{2}$ or NiEDDA were then determined from the difference in $\mathrm{P}_{1 / 2}$ values in the presence and absence of either $\mathrm{O}_{2}$ or NiEDDA, respectively. A depth parameter, $\Phi$, related to the local concentrations of $\mathrm{O}_{2}$ and NiEDDA, which vary as a function of depth in the lipid bilayer (Altenbach et al., 1994), was determined from the values of $\Delta \mathrm{P}_{1 / 2}$. The depth measurements were calculated for each $\Phi$ based on the calibration curve:

$$
\Phi=0.71403 * e^{x / 5.54055}-2.03138
$$

\subsection{CW-EPR Measurements}

Gltph was reconstituted into $400 \mathrm{~nm}$ vesicles, and $\mathrm{D}_{2} \mathrm{O}$ substrate free $(40 \mathrm{mM}$ HEPES, pH 7.4, $200 \mathrm{mM} \mathrm{KCl}), \mathrm{Na}^{+}$(40 mM HEPES, pH 7.4, $200 \mathrm{mM} \mathrm{NaCl}$ ), $\mathrm{Na}^{+} / \mathrm{Asp}$ (40 mM HEPES, pH 7.4, $200 \mathrm{mM} \mathrm{NaCl,} 2 \mathrm{mM} \mathrm{Na}{ }^{+}$/aspartate), or DL-TBOA buffer (40 mM HEPES, pH 7.4, 200 mM NaCl, 125 uM DL-TBOA (Tocris Bioscience, 
Avonmouth, Bristol, U.K.)) were added to the vesicles in a 1:1 v/v ratio. EPR spectra were recorded using a Bruker X-Band EMX spectrometer (Bruker Biospin, Billerica, MA) equipped with an ER 4123D dielectric resonator. All EPR spectra were recorded using a $100 \mathrm{G}$ magnetic field sweep, $1 \mathrm{G}$ modulation, and 2.0-milliwatt incident microwave power at a temperature of $298 \mathrm{~K}$. The measurements were performed on 10$\mu 1$ samples in glass capillary tubes $(0.60 \mathrm{~mm}$ inner diameter $\times 0.84 \mathrm{~mm}$ outer diameter round capillary; VitroCom, Mountain Lakes, NJ). The protein concentrations used were approximately $55 \mu \mathrm{M}$ of spin-labeled monomer. The phasing, normalization, and subtraction of EPR spectra were performed using LabVIEW software provided by Dr. Christian Altenbach (UCLA, Los Angeles, CA).

\subsection{Pulsed EPR measurements}

DEER samples containing $12.5 \mu \mathrm{L}$ of single-labeled Glt $\mathrm{p}_{\mathrm{ph}}$ in $20 \%$ deuterated glycerol were placed into quartz sample tubes having a $1.5 \mathrm{~mm}$ inner diameter and a $1.8 \mathrm{~mm}$ outer diameter (VitroCom, Mountain Lakes, NJ) and then rapidly frozen in liquid nitrogen. The data were recorded at $80 \mathrm{~K}$ on a dedicated Q-band Bruker E580 spectrometer fitted with an EN5107D2 dielectric resonator. For DEER experiments, the dead-time free four-pulse sequence with phase-cycled $\pi / 2$-pulse was used (Pannier et al., 2000). Typical pulse lengths were $18 \mathrm{~ns}$ for $\pi / 2$ and $36 \mathrm{~ns}$ for $\pi$ observe pulses and $36 \mathrm{~ns} \pi$ for the pump pulse. The pump pulse was set to the maximum of the echo-detected field swept spectrum, and the observer pulses were set $75 \mathrm{MHz}$ lower. The normalized primary DEER data were processed to remove the background function from intermolecular interactions, and the resulting form factors were fitted with a Tikhonov regularization with DeerAnalysis2013 to obtain distance distributions (Jeschke et al., 
2006). The error ranges shown in the distance distributions are based on variations in the background and dimensionality that produce fits within $15 \%$ of the root mean-square deviation (RMSD) of the best fits. Predictions of the expected distance distributions based on published crystal structures were made using the program Multiscale Modeling of Macromolecules (MMM) (Polyhatch et al., 2011), which used a rotamer library for the R1 side chain that was determined using density functional theory and consistent with available crystal structures (Warshaviak et al. 2011). Structures were visualized using the PyMOL Molecular Graphics System, Version 1.7.4 (Schrödinger). 
Chapter 8

References 
Akyuz, N.; Altman, R. B.; Blanchard, S. C.; Boudker, O. Transport Dynamics in a Glutamate Transporter Homologue. Nature, 2013, 502, 114-118.

Akyuz, N.; Georgieva, E. R.; Zhou, Z.; Stolzenberg, S.; Cuendet, M. A.; Khelashvili, G.; Altman, R. B.; Terry, D. S.; Freed, J. H.; Weinstein, H.; et al. Transport Domain Unlocking Sets the Uptake Rate of an Aspartate Transporter. Nature, 2015, 518, 68-73.

Altenbach, C.; López, C. J.; Hideg, K.; Hubbell, W. L. Exploring Structure, Dynamics, and Topology of Nitroxide Spin-Labeled Proteins Using Continuous-Wave Electron Paramagnetic Resonance Spectroscopy. Methods in Enzymology, 2015, 564, 59-100.

Altenbach, C.; Marti, T.; Khorana, H.; G.; Hubbell, W. L. Transmembrane Protein Structure: Spin Labeling of Bacteriorhodopsin Mutants. Science, 1990, 248, 1088-1092.

Arriza, J.L.; Eliasof, S.; Kavanaugh, M.P.; Amara, S.G. Excitatory Amino Acid Transporter 5, A Retinal Glutamate Transporter Coupled to a Chloride Conductance. PNAS., 1997, 94, 4155-4160.

Auton, M.; Rosgen, J.; Sinev, M.; Holthauzen, L. M.F.; Bolen, D.W. Osmolyte Effects on Protein Stability and Solubility: A Balancing Act Between Backbone and Side-Chains. Biophysical Chemistry, 2011, 159, 90-99.

Berliner, L.; Grunwald, J.; Hankovszky, H.O.; Hideg, K. A Novel Reversible Thiol Specific Spin Label: Papain Active Site Labeling and Inhibition. Analytical Biochemistry, 1982, 119, 450-455.

Bolen, D.; Baskakov, I.V. The Osmophobic Effect: Natural Selection of a Thermodynamic Force in Protein Folding. Journal of Molecular Biology, 2001, 310, 955-963.

Bolen, D.W. Protein Stabilization by Naturally Occurring Osmolytes. Methods in Molecular Biology, 2001, 168, 17-36.

Boudker, O.; Ryan, R.M.; Yernool, D.; Shimamoto, K.; Gouaux, E. Coupling Substrate and Ion Binding to Extracellular Gate of a Sodium-Dependent Aspartate Transporter. Nature, 2007, 445, 387-393.

Bridges, M.D.; Hideg, K.; Hubbell, W. L. Resolving Conformational and Rotameric Exchange in Spin-Labeled Proteins Using Saturation Recovery EPR. Applied Magnetic Resonance, 2010, 37, 363-377.

Brown, M.F. Curvature Forces in Membrane Lipid-Protein Interactions. Biochemistry, 2012, 51, 9782-9795. 
Budil, D. E.; Lee, S.; Saxena, S.; Freed, J. H. Nonlinear-Least-Squares Analysis of SlowMotion EPR Spectra in One and Two Dimensions Using a Modified LevenbergMarquardt Algorithm. Journal of Magnetic Resonance Series A, 1996, 120, 155-189.

Cafiso, D.S. Identifying and Quantitating Conformational Exchange in Membrane Proteins Using Site-Directed Spin Labeling. Accounts of Chemical Research, 2014, 47, 3102-3109.

Claxton, D.P.; Kazmier, K.; Mishra, S.; McHaourab, H.S. Navigating Membrane Protein Structure, Dynamics, and Energy Landscapes Using Spin Labeling and EPR Spectroscopy, 1st ed.; Elsevier Inc., 2015; Vol. 564.

Columbus, L.; Kálai, T.; Jekö, J.; Hideg, K.; Hubbell, W.L. Molecular Motion of Spin Labeled Side Chains in Alpha-Helices: Analysis by Variation of Side Chain Structure. Biochemistry, 2001, 40, 3828-3846.

Compton, E.L.R.; Taylor, E.M.; Mindell, J.A. The 3-4 Loop of an Archaeal Glutamate Transporter Homolog Experiences Ligand Induced Structural Changes and Is Important for Transport. PNAS, 2010, 107, 12840-12845.

Cross, T.A.; Murray, D.T.; Watts, A. Helical Membrane Protein Conformations and Their Environment. European Biophysics Journal, 2013, 42, 731-755.

Dalton, L.A.; McIntyre, J.O.; Fleischer, S. Distance Estimate of the Active Center of D$\beta$-Hydroxybutyrate Dehydrogenase from the Membrane Surface. Biochemistry, 1987, 26 , 2117-2130.

Erkens, G.B.; Hänelt, I.; Goudsmits, J.M.H.; Slotboom, D.J.; van Oijen, A.M. Unsynchronised Subunit Motion in Single Trimeric Sodium-Coupled Aspartate Transporters. Nature, 2013, 502, 119-123.

Ewers, D.; Becher, T.; Machtens, J.; Weyand, I.; Fahlke, C. Induced Fit Substrate Binding to an Archeal Glutamate Transporter Homologue. PNAS, 2013, 110, 1-6.

Fairman, W.A.; Vandenberg, R.J.; Arriza, J.L.; Kavanaugh, M.P.; Amara, S.G. An Excitatory Amino-Acid Transporter with Properties of a Ligand-Gated Chloride Channel. Nature, 1995, 375, 599-603.

Fanucci, G.E.; Lee, J.Y.; Cafiso, D.S. Spectroscopic Evidence that Osmolytes Used in Crystallization Buffers Inhibit a Conformation Change in a Membrane Protein.

Biochemistry, 2003, 42, 13106-13112. 
Flores Jiménez, R.H.; Do Cao, M.A.; Kim, M.; Cafiso, D.S. Osmolytes Modulate Conformational Exchange in Solvent-Exposed Regions of Membrane Proteins. Protein Science, 2010, 19, 269-278.

Focke, P.; Moenne-Locoz, P.; Larsson, H.P. Opposite Movement of the External Gate of a Glutamate Transporter Homologue Upon Binding Co-Transported Sodium Compared to Aspartate. Journal of Neuroscience, 2011, 31, 6255-6262.

Forrest L.R.; Zhang Y.W.; Jacobs M.T.; Gesmonde J.; Xie L.; Honig B.H.; Rudnick G. Mechanism for Alternating Access in Neurotransmitter Transporters. PNAS, 2008, 105, $10338-10343$.

Forrest, L.R.; Krämer, R.; Ziegler, C. The Structural Basis of Secondary Active Transport Mechanisms. Biochimica et Biophysica Acta, 2011, 1807, 167-188.

Frazier, A.A.; Wisner, M.A.; Malmberg, N.J.; Victor, K.G.; Fanucci, G.E.; Nalefski, E.A.; Falke, J.J; Cafiso, D.S. Membrane Orientation and Position of the C2 Domain from cPLA2 by Site-Directed Spin Labeling. Biochemistry, 2002, 41, 6282-6292.

Freed, D.M.; Horanyi, P.S., Wiener, M. C., \& Cafiso, D. S. Conformational Exchange in a Membrane Transport Protein is Altered in Protein Crystals. Biophysical Journal, 2010, 99, 1604-1610.

Freed, D.M.; Khan, A.K.; Horanyi, P.S.; Cafiso, D.S. Molecular Origin of EPR Lineshapes on $\beta$-Barrel Membrane Proteins: Local Solvation Environment Modulates Spin Label Configuration. Biochemistry, 2011, 50, 8792-8803.

Freed, J. H. Theory of Slow Tumbling ESR Spectra for Nitroxides. In Spin Labeling Theory and Applications, (ed. L. J. Berliner), 1976, pp. 53-132, Academic Press, New York.

Georgieva, E.R.; Borbat, P.P.; Ginter, C.; Freed, J.H.; Boudker, O. Conformational Ensemble of the Sodium-Coupled Aspartate Transporter. Nature Structural Molecular Biology, 2013, 20, 215-221.

Griffith, O. H.; Jost, P.C. Lipid Spin Labels in Biological Membranes. In Spin Labeling Theory and Applications, (ed. L. J. Berliner), 1976, pp. 454-523, Academic Press, New York.

Groeneveld, M.; Slotboom, D.J. Rigidity of the Subunit Interfaces of the Trimeric Glutamate Transporter GltT During Translocation. Journal of Molecular Biology, 2007, $372,565-570$. 
Gruner S.M. Intrinsic Curvature Hypothesis for Biomembrane Lipid Composition: A Role for Nonbilayer Lipids. PNAS., 1985, 82, 3665-3669.

Guo, Z.; Cascio, D.; Hideg, K.; Hubbell, W.L. Structural Determinants of Nitroxide Motion in Spin-Labeled Proteins: Solvent-Exposed Sites in Helix B of T4 Lysozyme. Protein Science, 2008, 17, 228-239.

Hänelt, I.; Jensen, S.; Wunnicke, D.,]; Slotboom, D.J. Low Affinity and Slow $\mathrm{Na}^{+}$ Binding Precedes High Affinity Aspartate Binding in the Secondary-active Transporter Glt Ph. Journal of Biological Chemistry, 2015, 290, 15962-15972.

Hänelt, I.; Wunnicke, D.; Bordignon, E.; Steinhoff, H.J.; Slotboom, D.J. Conformational Heterogeneity of the Aspartate Transporter GltPh. Nature Structural and Molecular Biology, 2013, 20, 210-214.

Harries, D.; Rosgen, J. A Practical Guide on How Osmolytes Modulate Macromolecular Properties. Methods in Cell Biology, 2008, 84, 679-735.

Hediger M.A.; Romero M.F.; Peng J.B.; Rolfs A.; Takanaga H.; Bruford E.A. The ABCs of Solute Carriers: Physiological, Pathological and Therapeutic Implications of Human Membrane Transport Proteins Introduction. Pflugers Archive European Journal of Physiology, 2004, 447, 465-468.

Hediger, M.A.; Clémençon B.; Burrier R.E.; Bruford E.A. The ABCs of Membrane Transporters in Health and Disease (SLC series): Introduction. Molecular Aspects of Medicine, 2013, 34, 95-107.

Hopkins, A. L.; Groom, C.R.; The Druggable Genome. Nature Reviews Drug Discovery, 2002, 1, 727-730.

Jardetzky O. Simple Allosteric Model for Membrane Pumps. Nature, 1996, 211, 969-70.

Jeschke, G.; Chechik, V.; Ionita, P.; Godt, A.; Zimmermann, H.; Banham, J.; Timmel, C.R.; Hilger, D.; Jung, H. DeerAnalysis2006 - A Comprehensive Software Package for Analyzing Pulsed ELDOR Data. Applied Magnetic Resonance, 2006, 30, 473-498.

Kanai, Y.; Clémençon, B.; Simonin, A.; Leuenberger, M.; Lochner, M.; Weisstanner, M.; Hediger, M.A. The SLC1 High-Affinity Glutamate and Neutral Amino Acid Transporter Family. Molecular Aspects of Medicine, 2013, 34, 108-120.

Kanai, Y.; Hediger, M.A. The Glutamate/Neutral Amino Acid Transporter Family SLC1: Molecular, Physiological and Pharmacological Aspects. Pflugers Archiv European Journal of Physiology, 2004, 447, 469-479. 
Kanner B.I.; Zomot E. Sodium-Coupled Neurotransmitter Transporters. Chemical Reviews, 2008 108, 1654-1668.

Ken, Q.; Pauisers, J.T. Comparative Analyses of Fundamental Differences in Membrane Transport Capabilities in Prokaryotes and Eukaryotes. PLoS Computational Biology, 2005, 1, 190-201.

Kivelson, D. Theory of ESR Linewidths of Free Radicals. Journal of Chemical Physics, 1960, 33, 1094-1106.

Krishnamurthy H.; Piscitelli C.L.; Gouaux E. Unlocking the Molecular Secrets of Sodium Coupled Transporters. Nature, 2009, 459, 347-355.

Kroncke, B.M.; Horanyi, P.S.; Columbus, L. Structural Origins of Nitroxide Side Chain Dynamics on Membrane Protein $\alpha$-Helical Sites. Biochemistry, 2010, 49, 10045-10060.

Larsson, H.P.; Wang, X.; Lev, B.; Baconguis, I.; Caplan, D.A.; Vyleta, N.P.; Noskov, S.Y. Evidence for a Third Sodium-Binding Site in Glutamate Transporters Suggests an Ion/Substrate Coupling Model. PNAS, 2010. 107, 13912-13917.

Lee, A.G. Biological Membranes: The Importance of Molecular Detail. Trends in Biochemical Science, 2011, 36, 493-500.

Levine, M.V.; Cuendet, M.A.; Khelashvili, G.; Weinstein, H. Allosteric Mechanisms of Molecular Machines at the Membrane: Transport by Sodium-Coupled Symporters. Chemical Reviews, 2016, 116, 6552-6587.

Lietzow, M.A.; Hubbell,W.L. Motion of Spin Label Side Chains in Cellular Retinol Binding Protein: Correlation with Structure and Nearest-Neighbor Interactions in an Antiparallel Beta-Sheet. Biochemistry, 2004, 43, 3137-3151.

Lin, L.; Yee, S.W.; Kim, R.B.; Giacomini, K.M. SLC Transporters as Therapeutic Targets: Emerging Opportunities. Nature Reviews Drug Discovery, 2015, 14, 543-560

Lodish H.; Berk A.; Zipursky S.L.; et al. Molecular Cell Biology, $4^{\text {th }}$ ed. New York: W. H. Freeman; 2000.

Lomize, M.A.; Lomize, A.L.; Pogozheva, I.D.; Mosberg, H.I. OPM: Orientations of Proteins in Membranes Database. Bioinformatics, 2006, 22, 623-625. 
Lopez, C.J.; Fleissner, M.R.; Guo, Z.; Kusnetzow, A.K.; Hubbell, W.L. Osmolyte Perturbation Reveals Conformational Equilibria in Spin-Labeled Proteins. Protein Science, 2009, 18, 1637-1652.

Marius, P.; Alvis, S.J.; East, J.M.; Lee, A.G. The interfacial lipid binding site on the potassium channel KcsA is specific for anionic phospholipids. Biophysical Journal, 2005. 89, 4081-4089.

Matchens, J.P.; Kortzak, D.; Lansche, C.; et al. Mechanisms of Anion Conduction by Coupled Glutamate Transporters. Cell, 2015, 160, 542-553.

Mchaourab, H.S.; Kalai, T.; Hideg, K.; Hubbell, W.L. Motion of Spin-Labeled Side Chains in T4 Lysozyme: Effect of Side Chain Structure. Biochemistry, 1999, 38, $2947-$ 2955.

Mchaourab, H.S.; Lietzow, M.A.; Hideg, K.; Hubbell, W.L. Motion of Spin-Labeled Side Chains in T4 Lysozyme. Correlation with Protein Structure and Dynamics. Biochemistry, 1996, 35, 7692-7704.

McIlwain, B. C.; Vandenberg, R.J.; Ryan, R.M. Transport Rates of a Glutamate Transporter Homologue Are Influenced by the Lipid Bilayer. Journal of Biological Chemistry, 2015, 290, 9780-9788.

Mouritsen O.G.; Bloom M. Mattress Model of Lipid-Protein Interactions in Membranes. Biophysical Journal, 1984, 46, 141-153.

Olaf, S.A.; Koeppe, R.E. Bilayer Thickness and Membrane Protein Function: An Energetic Perspective. Annual Reviews of Biophysics and Biomolecular Structure, 2007, $36,107-130$.

Pace, C.N.; Shaw, K.L. Linear Extrapolation Method of Analyzing Solvent Denaturation Curves. Proteins, 2000, 4(Suppl.), 1-7.

Palsdottir, H.; Hunte, C. Lipids in Membrane Protein Structures. Biochimica et Biophysica Acta, 2004. 1666, 2-18.

Pannier, M.; Veit, S.; Godt, A.; Jeschke, G.; Spiess, H.W. Dead-Time Free Measurement of Dipole-Dipole Interactions between Electron Spins. Journal of Magnetic Resonance, 2000, 142, 331-340.

Parsegian, V.A.; Rand, R.P.; Rau, D.C. Macromolecules and Water: Probing with Osmotic Stress. Methods in Enzymology, 1995, 259, 43-94. 
Polyhach, Y.; Bordignon, E.; Jeschke, G. Rotamer Libraries of Spin Labelled Cysteines for Protein Studies. Physical Chemistry Chemical Physics, 2011, 13, 2356-2366.

Powl, A.M.; East, J.M.; and Lee, A.G. Importance of Direct Interactions with Lipids for the Function of the Mechanosensitive Channel MscL. Biochemistry, 2008, 47, 1217512184.

Powl, A.M.; East, J.M.; Lee, A.G. Different Effects of Lipid Chain Length on the Two Sides of a Membrane and the Lipid Annulus of MscL. Biophysical Journal, 2007, 93, 113-122.

Prabhu, N.V.; Sharp, K.A. Heat Capacity in Proteins. Annual Review of Physical Chemistry, 2005, 56, 521-548.

Reyes, N.; Ginter, C.; Boudker, O. Transport Mechanism of a Bacterial Homologue of Glutamate Transporters. Nature, 2009, 462, 880-885.

Reyes, N.; Oh, S.; Boudker, O. Binding Thermodynamics of a Glutamate Transporter Homolog. Nature Structural and Molecular Biology, 2013, 20, 634-640.

Rothstein, J.D.; Dykes-Hoberg, M.; Pardo, C.A.; Bristol, L.A.; Jin, L.; Kuncl, R.W.; Kanai, Y.; Hediger, M.A.; Wang, Y.; Schielke, J.P.; Welty, D.F. Knockout of Glutamate Transporters Reveals a Major Role for Astroglial Transport in Excitotoxicity and Clearance of Glutamate. Neuron, 1996, 16, 675-686.

Rothstein, J.D.; Van Kammen, M.; Levey, A.I.,;Martin, L.J.; Kuncl, R.W. Selective Loss of Glial Glutamate Transporter GLT-1 in Amyotrophic Lateral Sclerosis. Annals of Neurology, 1995, 38, 73-84.

Ruan, Y.; Miyagi, A.; Wang, X.; Chami, M.; Boudker, O.; Scheuring, S. Direct Visualization of Glutamate Transporter Elevator Mechanism by High-Speed AFM. PNAS, 2016, 114, 1584-1588.

Ryan, R.M.; Compton, E.L.R.; Mindell, J.A. Functional Characterization of a Na+Dependent Aspartate Transporter from Pyrococcus Horikoshii. Journal of Biological Chemistry, 2009, 284, 17540-17548.

Ryan, R.M.; Mindell, J.A. The Uncoupled Chloride Conductance of a Bacterial Glutamate Transporter Homolog. Nature, 2007, 14, 365-371. 
Sahu, I.D.; Craig, A.F.; Dunagan, M.M.; Troxel, K.R.; Zhang, R.; Meiberg, A.G.; Harmon, C.N.; McCarrick, R.M.; Kroncke, B.M.; Sanders, C.R.; et al. Probing Structural Dynamics and Topology of the KCNE1 Membrane Protein in Lipid Bilayers via SiteDirected Spin Labeling and Electron Paramagnetic Resonance Spectroscopy.

Biochemistry, 2015, 54, 6402-641.

Saier, M.H. A Functional-Phylogenetic Classification System for Transmembrane Solute Screpanti E.; Hunte, C. Discontinuous membrane helices in transport proteins and their correlation with function. Journal of Structural Biology, 2007, 159, 261-267.

Shi, Y. Common Folds and Transport Mechanisms of Secondary Active Aransporters. Annual Reviews of Biophysics, 2013, 42, 51-72.

Smith, P. E. Equilibrium Dialysis Data and the Relationships Between Preferential Interaction Parameters for Biological Systems in Terms of Kirkwood-BuV Integrals. Journal of Physical Chemistry B Condensed Matter, Materials, Surfaces, Interfaces, and Biophysical, 2006b 110, 2862-2868.

Stansfeld, P.J.; Goose, J.E.; Caffrey, M.; Carpenter, E.P.; Parker, J.L.; Newstead, S.; Sansom, M.S. MemProtMD: Automated Insertion of Membrane Protein Structures into Explicit Lipid Membranes. Structure, 2015, 23, 1350-1361.

Steed, P. R.; Zou, P.; Trone, K. E.; McHaourab, H. S. Structure and pH-Induced Structural Rearrangements of the Putative Multidrug Efflux Pump EmrD in Liposomes Probed by Site-Directed Spin Labeling. Biochemistry, 2013, 52, 7964-7974.

Stevens, T.J.; Arkin, I.T.; The Effect of Nucleotide Bias upon the Composition and Prediction of Transmembrane Helices. Protein Sci. 2000, 9, 505-511.

Street, T.O.; Bolen, D.W.; Rose, G.D. A Molecular Mechanism for Osmolyte Induced Stabilization. PNAS, 2006, 13997-14002.

Sun, H.; Greathouse, D.V.; Andersen, O.S.; Koeppe, R.E. The Preference of Tryptophan for Membrane Interfaces: Insights from N-Methylation of Tryptophans in Gramicidin Channels. Journal of Biological Chemistry, 2008, 283, 22233-22243.

Tanford, C. The Location of Electrostatic Charges in Kirkwood's Model of Organic Ions. Journal of the American Chemical Society, 1957, 79, 5348-5352.

van den Brink-van der Laan, E.; Killian, J.A.; B. de Kruijff, Nonbilayer Lipids Affect Peripheral and Integral Membrane Proteins via Changes in the Lateral Pressure Profile. Biochimica et Biophysica Acta, 2004, 1666, 275-288. 
Van, S.H.; Birrell, B.; Griffith, O.H. Rapid Anisotropic Motion of Spin Labels. Models for Motion Averaging of the ESR Parameters. Journal of Magnetic Resonance, 1974, 15, 444-459.

Verdon, G.; Boudker, O. Crystal Structure of an Asymmetric Trimer of a Bacterial Glutamate Transporter Homolog. Nature Structural and Molecular Biology, 2012, 19, 355-357.

Verdon, G.; Oh, S.; Serio, R.; Boudker, O. Coupled Ion Binding and Structural Transitions along the Transport Cycle of Glutamate Transporters. eLife, 2014, 1-23.

Wadiche, J.I.; Amara, S.G.; Kavanaugh, M.P.; Ion Fluxes Associated with Excitatory Amino Acid Transport. Neuron, 1995a, 15, 721-728

Warshaviak, D.T.; Serbulea, L.; Houk, K.N.; Hubbell, W.L. Conformational Analysis of a Nitroxide Side Chain in an $\alpha$-Helix with Density Functional Theory. Journal of Physical Chemistry B, 2011, 115, 397-405.

Weber, R.T. Elexsys E580 Pulse EPR Spectrometer User's Manual. 2001. Whitelegge, J. P. Integral Membrane Proteins and Bilayer Proteomics. Analytical Chemistry, 2013, 85, 2558-2568.

Yernool, D.; Boudker, O.; Jin, Y.; Gouaux, E. Structure of a Glutamate Transporter Homologue from Pyrococcus Horikoshii. Nature, 2004, 431, 811-818.

Zerangue, N.; Kavanaugh, M.P.; ASCT-1 is a Neutral Amino Acid Exchanger with Chloride Channel Activity. Journal of Biological Chemistry, 1996b, 271, 27991-27994.

Zomot, E.; Bahar, I. Intracellular Gating in an Inward-Facing State of Aspartate Transporter GltPh Is Regulated by the Movements of the Helical Hairpin HP2. Journal of Biological Chemistry, 2013, 288, 8231-8237. 